\title{
Of void (vacuum) energy and quantum field : - a abstraction- subtraction model
}

\author{
${ }^{1}$ DR K N PRASANNA KUMAR, ${ }^{2}$ PROF B S KIRANAGI AND ${ }^{3}$ PROF C S \\ BAGEWADI \\ ${ }^{1}$ Post doctoral researcher, Dr KNP Kumar has three PhD's, one each in Mathematics, Economics and \\ Political science and a D.Litt. in Political Science, Department of studies in Mathematics, Kuvempu \\ University, Shimoga, Karnataka, India \\ ${ }^{2}$ UGC Emeritus Professor (Department of studies in Mathematics), Manasagangotri, University of Mysore, \\ Karnataka, India \\ ${ }^{3}$ Chairman, Department of studies in Mathematics and Computer science, Jnanasahyadri Kuvempu \\ university, Shankarghatta, Shimoga district, Karnataka, India
}

\begin{abstract}
A system of quantum field dissipating void and a parallel system of quantum field and void system that contribute to the dissipation of the velocity of void is investigated. It is shown that the time independence of the contributions portrays another system by itself and constitutes the equilibrium solution of the original time independent system. Methodology reinforced with the explanations, we write the governing equations with the nomenclature for the systems in the foregoing. Further papers extensively draw inferences upon such concatenation process, ipsofacto. Significantly consummation and consolidation of this model with that of the Grand Unified Theory is the one that results in the Quantum field giving rise to the basic forces which is purported to have been combined at the high temperatures at the Big Bang Vacuum energy is reported to be the reason for the consummation of the four forces at the scintillatingly high temperature.
\end{abstract}

\section{INTRODUCTION:}

Constitution ,Composition and outlay of the paper is expatiated in the following:

Review Of the Literature:

Under this head, we take an intimate and hawk's look at the various aspectionalities and attributions in the literature available. Vacuum Field is a subject which is a rarefied and moribund field. Vacuum Energy and Quantum Field combination and consummation, consolidation, concretization. Consubstantiation is a field which is rarely attempted to. Piece de resistance of the work is to put the study the concatenated formulated equations which has not been done earlier on terra firm. Under this head, in consideration to the fact that there are some Gordian Knots, we point out the extant and existential problems thereof. Additionally, expatiation, enucleation, elucidation and exposition of the points that are necessary for the formulation of the present problem are also notified. Here we study the aging process, dissipatory mechanism, obliteration, obfuscation and abjuration of the Vacuum energy and Quantum Field, with thrust on the problem solving capacity and state systemal and processual thinking on the subject matter.

\section{Work Suggested/Done:}

Under this appellation, we enumerate the work done, namely the sole aim, primary objective and sum mum bonuum of the work done. In the extant case we give the formulation of the problem. Statement of governing equations for both Vacuum energy and Quantum Field, write down in unmistakable terms the conceptual jurisprudence, phenomenological methodology, formal characterization, programmatic and anagrammatic concatenation of the equations. We discuss in detail for the system Vacuum Energy and Quantum Field, the stability analysis, solution behavior, asymptotic analysis, the three formidable but very important tools for the system to remain as sangfroid like salamander under various conditionalities or undergo transformation with environmental decoherence. This aspect throws light on hither to untouched regions of Quark similarity, Schwarzschild radius, Zero Point Energy, Quantum Chromo Dynamics, GTR, STR,Quarks,Gluons,,and the concomitant and corresponding accentuatory, corroboratory 
,augmentory or dissipatory relationship. As is stated in the foregoing, these factors are very important for the model to be put as a promethaleon, primogeniture and proponent for further study which the author intends to do. These constituent structures, transformational minimal conditions, structural morphology, dependent variability, normative aspect of expectations from the model are discussed. We give a prediction model for the system VE-QF and derive stability analysis, asymptotic stability and solutional behavior of the system.

\section{Review Of the Literature: PHYSICS AND VOID}

The first observation that we make the moment we set out to study the phenomenon called "space" is that the adjective "empty" can never accurately be applied to it. Since we have already found that the energy fields we call "matter" are all interconnected by other fields, which we call "forces," there cannot be even the tiniest area of space anywhere in the cosmos in which at least one of these field-types is not present.

According to relativity theory, however, an energy field does not just fill the area of space, which contains it - the field actually is the space itself. Field=Space itself. An energy field determines the actual structure of the space it inhabits; The consequences of this discovery become far-reaching indeed, when we remember that matter is nothing but a particular type of energy field, for this means that matter and space must now be considered inseparable and interdependent. Modern physics considers matter and space to be two different aspects of a single phenomenon. This explains why the closer we look at a material particle; the more all distinctions between it and the space around it become lost. It also accounts for the astounding fact that such particles can be observed actually springing into existence directly from the void, as well as vanishing suddenly back into nothingness. What we are seeing are simply two different sides of the same coin.

The reigning model of the universe upheld by physicists today is that of quantum field theory, which effectively combines the laws of classical physics with those of quantum theory and relativity. This view of the cosmos erases all distinctions between matter, energy and space, encompassing them all within a single physical reality called the quantum field. This field is present everywhere, and its most distinctive characteristic is that there are two apparent aspects to its basic nature: (1) it has a continuous structure which we know as "space" and "time" because this aspect seems to exist constantly and changelessly throughout the cosmos and also throughout the past, present and future; and (2) it also has a granular or particle aspect which we know as "matter" and "force" because in this aspect the quantum field appears in the form of discontinuous, localized particles which enjoy only temporary existence. The field continually oscillates between these two apparently dissimilar states, incessantly transforming itself from one to the other.

Now we can no longer consider space to be simply a static background or events or a passive container of objects, for it does in tact possess a vital, self-governing structure of its own. If we want to really understand the nature of a subatomic particle, we must observe it in connection with the space around it, instead of trying to draw distinctions between the particle and that space.

Observed in this light, for example, an electron reveals itself to be a kind of "energy knot," a blemish on the face of its underlying field. It does not in fact revolve around its nucleus as does our earth around its sun, instead, it propagates through space like a water wave, and clearly does not consist of any selfsame substance at all times. A material particle, it turns out, is simply a local condensation of the quantum field, a temporary concentration of energy which appears solid to our sight and touch. In the words of Albert Einstein: "We may therefore regard matter as being constituted by the regions of space in which the field is extremely intense... . There is no place in this kind of physics both for the field and matter, for the field is the only reality."

The physical vacuum, as the void is called in quantum field theory, is far from empty nothingness, for it contains an infinite number of every type of particle in potential form, which also indirectly means that it contains all material objects in potential form as well. Every object in the world around us is a transient particle manifestation of the quantum field, and every interaction among these objects, as well as between us and them, is also carried by this field in the form of vibrational waves. If it were not for this living, moving void, if it did not continually vibrate in an endless dance of creation and destruction, there would be no physical universe, no perceptible reality at all, anywhere. The discovery that the physical vacuum or void is alive and active is one of the outstanding revelations of modem physics.

As yet, science does not know what the quantum field is made of, but it is now considered the fundamental substance of all material phenomena in Creation. It is not, however, viewed as the basis of all 
nonmaterial phenomena, most notably the force of gravity. Convinced that there must exist an even more fundamental ground field which would prove to be the basis of both the quantum and gravitational fields, Einstein spent twenty years of his life in pursuit of such a unified field theory. Finally, in 1949, he presented a mathematical solution which brings these apparently diverse phenomena under the same set of equations, but three decades later his theory remains unverified, since no practicable way has yet been found to confirm the results of his mathematics with experimental evidence.

\section{What Lies Beneath the Void}

Three thousand years ago, the Greek philosophers Leucippus and his student Democritus proposed the concept of the atom, as a fundamental building block of materials, in order to circumvent a paradox that arises with continuous elements (such as earth fire air and water). They pointed out that if matter was really a continuum then you could cut it into smaller and smaller pieces ad infinitum and, in principle, cut it out of existence into pieces of nothing that could not then be reassembled. Thus, they reasoned, there must be a smallest piece of matter that could not be further divided the a-tomon (uncuttable) from which the word atom is derived. To complete the picture you also need a void in which the atoms move, a concept that produced fervent debate, for example, is the void a 'nothing' or a 'something' and is it a continuum or does the void itself have an uncuttable smallest unit.

While the atom, the legacy of Leucippus and Democritus, is now a familiar part of the scientific landscape, the true nature of the void remains a mystery. In classical Physics, the void is a 'nothing', a simple absence of all matter and energy. Quantum theory tells a different story and states that the void is definitely a 'something'. It is a seething mass of 'virtual' particles that fleetingly appear into and then disappear from our observable universe. This activity, known as quantum fluctuations, corresponds to an intrinsic energy of the void, the 'zero-point energy', which, if the void were a continuum, would be infinite. It is generally believed that there is a smallest piece of void, which makes the zero-point energy finite but still colossal beyond the imagination. Each cubic millimeter of empty space contains more than enough zeropoint energy to create a new universe.

In a sense, the actual value of the zero-point energy is not important because everything we know about is on top of it. According to quantum field theory, every particle is an excitation (a wave) of an underlying field (for example the electromagnetic field) in the void and it is only the energy of the wave itself that we can detect. A useful analogy is to consider our observable universe as a mass of waves on top of an ocean, whose depth is immaterial. Our senses and all our instruments can only directly detect the waves so it seems that trying to probe whatever lies beneath, the void itself, is hopeless. Not quite so. There are subtle effects of the zero-point energy that do lead to detectable phenomena in our observable universe. An example is a force, predicted in 1948 by the Dutch physicist, Hendrik Casimir that arises from the zero point energy. If you place two mirrors facing each other in empty space, they produce a disturbance in the quantum fluctuations that results in a pressure pushing the mirrors together. Detecting the Casimir force however is not easy as it only becomes significant if the mirrors approach to within less than 1 micrometer (about a fiftieth the width of a human hair). Producing sufficiently parallel surfaces to the precision required had to wait for the emergence of the tools of nanotechnology to make accurate measurements of the force.

In the last decade this has happened and a spate of measurements using atomic force microscopes has confirmed Casmir's prediction to a precision of about 5\% and the zero-point energy of the void is an experimental reality. This is just the beginning however as the force has only been measured in very simple geometries such as flat parallel plates. More recent calculations show that the force is sensitive to geometry and by changing the materials and the shape of the cavity you can alter the magnitude of the Casimir force and possibly even reverse it. This would be a groundbreaking discovery as the Casimir force is a fundamental property of the void and reversing it is akin to reversing gravity. Technologically this would only have relevance at very small distances but it would revolutionize the design of micro- and nano-machines.

The srif2 and srif3 investment by the University of Leicester in the Virtual Microscopy Centre and the Nanoscale Interfaces Centre has put the University in a key position to take a lead in Casimir force measurements in novel geometries. Casimir force measurements in non-simple cavities and assess the utility of the force in providing a method for contactless transmission in nano-machines.

This new wave of measurements will enable an unprecedented level of probing of the void, will provide important information on new theories of gravity, and with sufficient precision will even put limits on the true number of spatial dimensions. Knowing how zero-point energy varies with the shape of an enclosure 
may also give clues to the origin of so-called 'dark energy', discovered recently. (Excerpts from lectures of Professor Chris Binns, Physics and Astronomy)

\section{VACUUM ENERGY}

Vacuum energy is an underlying background energy that exists in space even when the space is devoid of matter (free space). The concept of vacuum energy has been deduced from the concept of virtual particles, which is itself derived from the energy-time uncertainty principle. The effects of vacuum energy can be experimentally observed in various phenomena such as spontaneous emission, the Casimir effect, the van der Waals bonds and the Lamb shift, and are thought to influence the behavior of the Universe on cosmological scales. Using the upper limit of the cosmological constant, the vacuum energy in a cubic meter of free space has been estimated to be $10^{-9}$ Joules. However, in both Quantum Electrodynamics (QED) and Stochastic Electrodynamics (SED), consistency with the principle of Lorentz covariance and with the magnitude of the Planck Constant requires it to have a much larger value of $10^{113}$ Joules per cubic meter.

\section{Origin}

Quantum field theory states that all fundamental fields, such as the electromagnetic field, must be quantized (possible age wise classifications scheme applicable)at each and every point in space. A field in physics may be envisioned as if space were filled with interconnected vibrating balls and springs, and the strength of the field were like the displacement of a ball from its rest position. The theory requires "vibrations" in, or more accurately changes in the strength of, such a field to propagate as per the appropriate wave equation for the particular field in question. The second quantization of quantum field theory requires that each such ball-spring combination be quantized, that is, that the strength of the field be quantized at each point in space. Canonically, if the field at each point in space is a simple harmonic oscillator, its quantization places a quantum harmonic oscillator at each point. Excitations of the field correspond to the elementary particles of particle physics. Thus, according to the theory, even the vacuum has a vastly complex structure and all calculations of quantum field theory must be made in relation to this model of the vacuum.

The theory considers vacuum to implicitly have the same properties as a particle, such as spin or polarization in the case of light, energy, and so on. According to the theory, most of these properties cancel out on average leaving the vacuum empty in the literal sense of the word. One important exception, however, is the vacuum energy or the vacuum expectation value of the energy. The quantization of a simple harmonic oscillator requires the lowest possible energy, or zero-point energy of such an oscillator to be:

$$
E=\frac{1}{2} \hbar \omega \text {. }
$$

Summing over all possible oscillators at all points in space gives an infinite quantity. This is most important point. Vacuum energy is summable.To remove this infinity; one may argue that only differences in energy are physically measurable, much as the concept of potential energy has been treated in classical mechanics for centuries. This argument is the underpinning of the theory of renormalization. In all practical calculations, this is how the infinity is handled.

Vacuum energy can also be thought of in terms of virtual particles (also known as vacuum fluctuations) which are created and destroyed out of the vacuum. Creation and destruction of virtual particles (e) Vacuum energy. These virtual particles are always created out of the vacuum in particleantiparticle pairs, which in most cases shortly annihilate each other and disappear. However, these particles and antiparticles may interact with others before disappearing, a process which can be mapped using Feynman diagrams. Note that this method of computing vacuum energy is mathematically equivalent to having a quantum at each point and, therefore, suffers the same renormalization problems. Additional contributions to the vacuum energy come from spontaneous symmetry breaking in quantum field theory.

\section{IMPLICATIONS}

Vacuum energy has a number of consequences. In 1948, Dutch physicists Hendrik B. G. Casimir and Dirk Polder predicted the existence of a tiny attractive force between closely placed metal plates due to resonances in the vacuum energy in the space between them. This is now known as the Casimir 
effect and has since been extensively experimentally verified. It is therefore believed that the vacuum energy is "real" in the same sense that more familiar conceptual objects such as electrons, magnetic fields, etc., are real. However, the Casimir effect is no certain proof for vacuum energy since it can also be explained without this theory.

Other predictions are harder to verify. Vacuum fluctuations are always created as particle/antiparticle pairs. The creation of these virtual particles near the event horizon of a hole has been hypothesized by physicist Stephen Hawking to be a mechanism for the eventual "evaporation" of black holes. The net energy of the Universe remains zero so long as the particle pairs annihilate each other within Planck time. If one of the pair is pulled into the black hole before this, then the other particle becomes "real" and energy/mass is essentially radiated into space from the black hole. This loss is cumulative and could result in the black hole's disappearance over time. The time required is dependent on the mass of the black hole but could be on the order of $10^{100}$ years for large solar-mass black holes.

The vacuum energy also has important consequences for physical cosmology. Special relativity predicts that energy is equivalent to mass, and therefore, if the vacuum energy is "really there", it should exert a gravitational force. Essentially, a non-zero vacuum energy is expected to contribute to the cosmological constant, which affects the expansion of the universe. In the special case of vacuum energy, general relativity stipulates that the gravitational field is proportional to $\rho-3 p$ (where $\rho$ is the massenergy density, and $p$ is the pressure). Quantum theory of the vacuum further stipulates that the pressure of the zero-state vacuum energy is always negative and equal to $\rho$. Thus, the total of $\rho-3 p$ becomes $-2 \rho$ : A negative value. This calculation implies a repulsive gravitational field, giving rise to expansion, if indeed the vacuum ground state has non-zero energy. However, the vacuum energy is mathematically infinite without renormalization, which is based on the assumption that we can only measure energy in a relative sense, which is not true if we can observe it indirectly via the cosmological constant

\section{VIRTUAL PARTICLE-FIELDS OF BASIC FORCE INTERACTIONS}

In physics, a virtual particle is a particle that exists for a limited time and space. The energy and momentum of a virtual particle are uncertain according to the uncertainty principle. The degree of uncertainty of each is inversely proportional to time duration (for energy) or to position span (for momentum).

Virtual particles exhibit some of the phenomena that real particles do, such as obedience to the conservation laws. If a single particle is detected, then the consequences of its existence are prolonged to such a degree that it cannot be virtual. Virtual particles are viewed as the quanta that describe fields of the basic force interactions, which cannot be described in terms of real particles. Examples of these are static force fields, such as a simple electric or magnetic field, or the components of any field that do not carry information from place to place at the speed of light (information radiated by means of a field must be composed of real particles). Virtual photons are also a major component of antenna near field phenomena and induction fields, which have shorter-range effects, and do not radiate through space with the same rangeproperties as do electromagnetic wave photons. For example, the energy carried from one winding of a transformer to another, or to and from a patient in an MRI scanner, in quantum terms is carried by virtual photons, not real photons.

The virtual particle forms of massless particles, such as photons, do have mass (which may be either positive or negative) and are said to be off mass shell. They are allowed to have mass (which consists of "borrowed energy" because they exist for only a temporary time, which in turn gives them a limited "range". This is in accordance with the uncertainty, which allows existence of such particles of borrowed energy, so long as their energy, multiplied by the time they exist, is a fraction of Planck's constant. Possession of mass also allows single virtual photons to be more easily created and emitted from single charged elementary particles, something that cannot happen for massless photons, without violating conservation of momentum and energy (single real photons are always created and emitted from systems of two or more particles). For particles that do have a rest mass, their virtual forms still violate the energy-momentum relation of special relativity, in having a mass more or less than predicted by the relation $\quad E^{2}-p^{2} c^{2}=m^{2} c^{4}$. For this reason, the force-carrier particles are generally massless - the primary exception being the $\mathrm{W}^{+/-}$and $\mathrm{Z}^{[2]}$ bosons of the Weak Interaction. The concept of virtual particles is closely related to the idea of quantum 
fluctuations. Virtual particles can be thought of as coming into existence as quantities, such as the electric field, which fluctuate around their expectation values as required by quantum mechanics. The concept of virtual particles arises in the perturbation theory of quantum field theory, an approximation scheme in which interactions (in essence, forces) between real particles are calculated in terms of exchanges of virtual particles. Any process involving virtual particles admits a schematic representation known as a Feynman diagram, which facilitates the understanding of calculations.

A virtual particle is one that does not precisely obey the $\mathrm{m}^{2} \mathrm{c}^{4}=E^{2}-\mathrm{p}^{2} \mathrm{c}^{2}$ relationship for a short time. In other words, its kinetic energy may not have the usual relationship to velocity-indeed, it can be negative. The probability amplitude for it to exist tends to be canceled out by destructive interference over longer distances and times. A virtual particle can be considered a manifestation of quantum tunneling. The range of forces carried by virtual particles is limited by the uncertainty principle, which regards energy and time as conjugate variables; thus, virtual particles of larger mass have more limited range.

There is not a definite line differentiating virtual particles from real particles - the equations of physics just describe particles (which includes both equally). The amplitude that a virtual particle exists interferes with the amplitude for its non-existence, whereas for a real particle the cases of existence and nonexistence cease to be coherent with each other and do not interfere any more. In the quantum field theory view, "real particles" are viewed as being detectable excitations of underlying quantum fields. As such, virtual particles are also excitations of the underlying fields, but are detectable only as forces but not particles. They are "temporary" in the sense that they appear in calculations, but are not detected as single particles. Thus, in mathematical terms, they never appear as indices to the scattering matrix, which is to say, they never appear as the observable inputs and outputs of the physical process being modeled. In this sense, virtual particles are an artifact of perturbation theory, and do not appear in a non-perturbative treatment.

There are two principal ways in which the notion of virtual particles appears in modern physics. They appear as intermediate terms in Feynman diagrams; that is, as terms in a perturbative calculation. They also appear as an infinite set of states to be summed or integrated over in the calculation of a semi-nonperturbative effect. In the latter case, it is sometimes said that virtual particles cause the effect, or that the effect occurs because of the existence of virtual particles.

\section{MANIFESTATIONS}

There are many observable physical phenomena resulting from interactions involving virtual particles. For bosonic particles that exhibit rest mass when they are free and "real," virtual interactions are characterized by the relatively short range of the force interaction produced by particle exchange. Examples of such short-range interactions are the strong and weak forces, and their associated field bosons. For the gravitational and electromagnetic forces, the zero rest-mass of the associated boson particle permits long-range forces to be mediated by virtual particles. However, in the case of photons, power and information transfer by virtual particles is a relatively short-range phenomenon (existing only within a few wavelengths of the field-disturbance, which carries information or transferred power), as for example seen in the characteristically short range of inductive and capacitative effects in the near field zone of coils and antennas. Some field interactions which may be seen in terms of virtual particles are:

The Coulomb force (static electric force) between electric charges. It is caused by the exchange of virtual photons. In symmetric 3-dimensional space this exchange results in the inverse for electric force. Since the photon has no mass, the coulomb potential has an infinite range.

The magnetic field between magnetic dipoles is caused by the exchange of virtual photons. In symmetric 3-dimensional space this exchange results in the inverse square law for magnetic force. Since the photon has no mass, the magnetic potential has an infinite range. Much of the so-called near-field of radio antennas, where the magnetic and electric effects of the changing current in the antenna wire and the charge effects of the wire's capacitive charge may be (and usually are) important contributors to the total EM field close to the source, but both of which effects are dipole effects that decay with increasing distance from the antenna much more quickly than do the influence of "conventional" electromagnetic waves that are "far" from 
the source. ["Far" in terms of terms of ratio of antenna length or diameter, to wavelength]. These far-field waves, for which $\mathbf{E}$ is (in the limit of long distance) equal to $\mathbf{c B}$, are composed of real photons. It should be noted that real and virtual photons are mixed near an antenna, with the virtual photons responsible only for the "extra" magnetic-inductive and transient electric-dipole effects, which cause any imbalance between $\mathrm{E}$ and $\mathrm{cB}$. As distance from the antenna grows, the near-field effects (as dipole fields) die out more quickly, and only the "radiative" effects that are due to real photons remain as important effects. Although virtual effects extend to infinity, they drop off in field strength as $1 / \mathrm{r}^{2}$ rather than the field of EM waves composed of real photons, which drop $1 / \mathrm{r}$ (the powers, respectively, decrease as $1 / \mathrm{r}^{4}$ and $1 / \mathrm{r}^{2}$ ). See near and far field for a more detailed discussion. See near field communication for practical communications applications of near fields.

Electromagnetic induction. This phenomenon transferring energy to and from a magnetic coil via a changing (electro)magnetic field can be viewed as a near-field effect. It is the basis for power transfer in transformers and electric generators and motors, and also signal transfer in metal detectors, magnetic and magnetoacustic anti theft electronic tags, and even signals between patient and machine in an MRI scanner. Some confusion about the use of "radio waves" results when these devices are used at conventional RF frequencies, as they are in an MRI scanner. See resonant inductive coupling and wireless energy transfer for other practical examples.

The strong nuclear force between quarks is the result of interaction of virtual gluons. The residual of this force outside of quark triplets (neutron and proton) holds neutrons and protons together in nuclei, and is due to virtual mesons such as the pi meson and rho meson. The weak nuclear force - it is the result of exchange by virtual bosons. There is spontaneous emission of a photon during the decay of an excited atom or excited nucleus; such decay is prohibited by ordinary quantum mechanics and requires the quantization of the electromagnetic field for its explanation.

The Casimir effect, where the ground state of the quantized electromagnetic field causes attraction between a pair of electrically neutral metal plates. The van der Waals force, which is partly due to the Casimir effect between two atoms. Vacuum polarization, which involves pair production or the decay of the vacuum, which is the spontaneous production of particle-antiparticle pairs (such as electron-positron).Lamb shift of positions of atomic levels. Hawking radiation, where the gravitational field is so strong that it causes the spontaneous production of photon pairs (with black body energy distribution) and even of particle pairs.

Most of these have analogous effects in solid-state physics; indeed, one can often gain a better intuitive understanding by examining these cases. In semiconductors, the roles of electrons, positrons and photons in field theory are replaced by electrons in the conduction band, holes in the valence band, and phonons or vibrations of the crystal lattice. A virtual particle is in a virtual state where the probability amplitude is not conserved (not constant). Examples of macroscopic virtual phonons, photons, and electrons in the case of the tunneling process were presented by Günter Nimtz Paul Dirac was the first to propose that empty space (a vacuum) can be visualized as consisting of a sea of virtual electron-positron pairs, known as the Dirac Sea. The Dirac Sea has a direct analog to the electronic band structure in crystalline solids as described in solid state physics. Here, particles correspond to conduction electrons, and antiparticles correspond to to holes. A variety of interesting phenomena can be attributed to this structure.

Virtual particles in Feynman diagrams

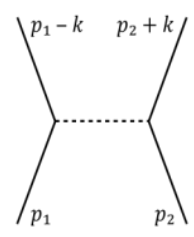

One particle exchange scattering diagram

The calculation of scattering amplitudes in theoretical particle physics requires the use of some rather large and complicated integrals over a large number of variables. These integrals do, however, have a 
regular structure, and may be represented as Feynman diagrams. The appeal of the Feynman diagrams is strong, as it allows for a simple visual presentation of what would otherwise be a rather arcane and abstract formula. In particular, part of the appeal is that the outgoing legs of a Feynman diagram can be associated with real, on-shell particles. Thus, it is natural to associate the other lines in the diagram with particles as well, called the "virtual particles". In mathematical terms, they correspond to the propagators appearing in the diagram.

In the image to the right, the solid lines correspond to real particles (of momentum $\mathrm{p}_{1}$ and so on), while the dotted line corresponds to a virtual particle carrying momentum $k$. For example, if the solid lines were to correspond to electrons interacting by means of the electromagnetic interaction, the dotted line would correspond to the exchange of a virtual photon. In the case of interacting nucleons, the dotted line would be a virtual pion. In the case of quarks interacting by means of the strong force, the dotted line would be a virtual gluon, and so on.

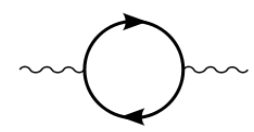

It is sometimes said that all photons are virtual photons. This is because the world-lines of photons always resemble the dotted line in the above Feynman diagram: The photon was emitted somewhere (say, a distant star), and then is absorbed somewhere else (say a photoreceptor cell in the eyeball). Furthermore, in a vacuum, a photon experiences no passage of (proper) time between emission and absorption. This statement illustrates the difficulty of trying to distinguish between "real" and "virtual" particles, because, in mathematical terms, they are the same objects and it is only our definition of "reality" that is weak here. In practice, a clear distinction can be made: real photons are detected as individual particles in particle detectors, whereas virtual photons are not directly detected; only their average or side-effects may be noticed, in the form of forces or (in modern language) interactions between particles.

Virtual particles may be mesons or vector bosons, as in the example above; they may also be fermions. However, in order to preserve quantum numbers, most simple diagrams involving fermion exchange are prohibited. The image to the right shows an allowed diagram, a one-loop diagram. The solid lines correspond to a fermion propagator, the wavy lines to bosons.

\section{VIRTUAL PARTICLES IN VACUUMS}

In formal terms, a particle is considered to be an eigenstate of the particle number operator $\mathrm{a}^{\dagger} \mathrm{a}$, where $\mathrm{a}$ is the particle annihilation operator and $\mathrm{a}^{\dagger}$ the particle creation operator (sometimes collectively called ladder operators Kawaguchi likened them to Brahma and Vishnu and Maheshwara in Hindu mythology)). In many cases, the particle number operator does notcommute with the Hamiltonian for the system. This implies the number of particles in an area of space is not a well-defined quantity but, like other quantum observables, is represented by a probability distribution. Since these particles do not have a permanent existence, they are called virtual particles or vacuum fluctuations of vacuum energy. In a certain sense, they can be understood to be a manifestation of the time-energy uncertainty principle in a vacuum. ${ }^{\text {I }}$

An important example of the "presence" of virtual particles in a vacuum is the Casimir effect. Here, the explanation of the effect requires that the total energy of all of the virtual particles in a vacuum can be added together. Thus, although the virtual particles themselves are not directly observable in the laboratory, they do leave an observable effect: Their zero-point energy results in forces acting on suitably arranged metal plates or dielectrics.

\section{PAIR PRODUCTION}

In order to conserve the total fermion number of the universe, a fermion cannot be created without also creating its antiparticle; thus, many physical processes lead to pair creation. The need for the normal ordering of particle fields in the vacuum can be interpreted by the idea that a pair of virtual particles may briefly "pop into existence", and then annihilate each other a short while later.

Thus, virtual particles are often popularly described as coming in pairs, a particle and antiparticle, which can 
be of any kind. These pairs exist for an extremely short time, and mutually annihilate in short order. In some cases, however, it is possible to boost the pair apart using external energy so that they avoid annihilation and become real particles. This may occur in one of two ways. In an accelerating frame of reference, the virtual particles may appear to be real to the accelerating observer; this is known as the Unruh effect. In short, the vacuum of a stationary frame appears, to the accelerated observer, to be a warm gas of real particles in thermodynamic equilibrium. The Unruh effect is a toy model for understanding Hawking radiation, the process by which black holes evaporate.

Another example is pair production in very strong electric fields, sometimes called vacuum decay. If, for example, a pair of atomic nuclei are merged together to very briefly form a nucleus with a charge greater than about 140, (that is, larger than about the inverse of the fine structure constant, which is a dimensionless quantity), the strength of the electric field will be such that it will be energetically favorable to create positron-electron pairs out of the vacuum or Dirac sea, with the electron attracted to the nucleus to annihilate the positive charge. This pair-creation amplitude was first calculated by Julian Schwinger. The restriction to particle-antiparticle pairs is actually only necessary if the particles in question carry a conserved quantity, such as electric charge, which is not present in the initial or final state. Otherwise, other situations can arise. For instance, the beta decay of a neutron can happen through the emission of a single virtual, negatively charged $\mathrm{W}$ particle that almost immediately decays into a real electron and antineutrino; the neutron turns into a proton when it emits the $\mathrm{W}$ particle. The evaporation of a black hole is a process dominated by photons, which are their own antiparticles and are uncharged.

\section{THE QCD VACUUM STATE}

The QCD vacuum is the vacuum state of quantum chromodynamics (QCD). It is an example of a non-perturbative vacuum state, characterized by many non-vanishing condensates such as the gluon condensate or the quark condensate. These condensates characterize the normal phase or the confined phase of quark matter. $Q C D$ in the non-perturbative regime: confinement. The equations of QCD remain unsolved at energy scales relevant for describing atomic nuclei. How does QCD give rise to the physics of nuclei and nuclear constituents?

\section{Symmetries and symmetry breaking}

\section{Symmetries of the QCD Lagrangian}

Like any relativistic quantum field theory, QCD enjoys Poincare symmetry including the discrete symmetries CPT (each of which is realized). Apart from these space-time symmetries, it also has internal symmetries. Since QCD is an SU (3) gauge theory, it has local SU (3) symmetry. Since it has many flavors of quarks, it has approximate flavor and chiral symmetry. This approximation is said to involve the chiral limit of QCD. Of these chiral symmetries, the baryon symmetry is exact. Some of the broken symmetries include the axial $\mathrm{U}(1)$ symmetry of the flavor group. This is broken by the chiral anomaly. The presence of instantons implied by this anomaly also breaks $\mathrm{CP}$ symmetry.

In summary, the QCD Lagrangian has the following symmetries:

Poincare symmetry and CPT invariance

- $\mathrm{SU}(3)$ local gauge symmetry

- approximate global $\mathrm{SU}\left(\mathrm{N}_{\mathrm{f}}\right) \mathrm{XSU}\left(\mathrm{N}_{\mathrm{f}}\right)$ flavour chiral symmetry and the $\mathrm{U}(1)$ baryon number symmetry

The following classical symmetries are broken in the QCD Lagrangian: scale, i.e., conformal symmetry (through the scale anomaly), giving rise to freedom the axial part of the U(1) flavour chiral symmetry (through the chiral anomaly), giving rise to the strong CP problem.

\section{Spontaneous symmetry breaking}

When the Hamiltonian of a system (or the Lagrangian) has certain symmetry, but the ground state (i.e., the vacuum) does not, then one says that spontaneous symmetry breaking (SSB) has taken place.

A familiar example of SSB is in ferromagnetic materials. Microscopically, the material consists www.iosrjournals.org 
of atoms with a non-vanishing spin, each of which acts like a tiny bar magnet, i.e., magnetic. The Hamiltonian of the material, describing the interaction of neighboring dipoles, is invariant under rotations. At high temperature, there is no magnetization of a large sample of the material. Then one says that the symmetry of the Hamiltonian is realized by the system. However, at low temperature, there could be an overall magnetization. This magnetization has a preferred direction, since one can tell the north magnetic pole of the sample from the south magnetic pole. In this case, there is spontaneous symmetry breaking of the rotational symmetry of the Hamiltonian. When a continuous symmetry is spontaneously broken, massless bosons appear, corresponding to the remaining symmetry. This is called the Goldstone phenomenon and the bosons are called Goldstone bosons.

\section{Symmetries of the $Q C D$ vacuum}

The $\mathrm{SU}\left(\mathrm{N}_{\mathrm{f}}\right) \times \mathrm{SU}\left(\mathrm{N}_{\mathrm{f}}\right)$ chiral flavour symmetry of the QCD Lagrangian is broken in the vacuum state of the theory. The symmetry of the vacuum state is the diagonal $\mathrm{SU}\left(\mathrm{N}_{\mathrm{f}}\right)$ part of the chiral group. The diagnostic for this is the formation of a non-vanishing chiral condensate $\left\langle\bar{\psi}_{i} \psi_{i}\right\rangle$, where $\psi_{\mathrm{i}}$ is the quark field operator, and the flavour index $\mathbf{i}$ is summed. The Goldstone bosons of the symmetry breaking are the pseudoscalar mesons. When $\mathrm{N}_{\mathrm{f}}=2$, i.e., only the $\mathbf{u}$ and $\mathbf{d}$ quarks are treated as massless, the three pions are the Goldstone bosons. When the $\mathbf{s}$ quark is also treated as massless, i.e., $\mathrm{N}_{\mathrm{f}}=3$, all eight pseudoscalar mesons of the quark model become Goldstone bosons. The actual masses of these mesons are obtained in chiral perturbation theory through an expansion in the (small) actual masses of the quarks. In other phases of quark matter the full chiral flavour symmetry may be recovered, or broken in completely different ways.

\section{Evidence: experimental consequences}

The evidence for QCD condensates comes from two eras, the pre-QCD era 1950-1973 and the postQCD era, after 1974. The pre-QCD results established that the strong interactions vacuum contains a quark chiral condensate, while the post-QCD results established that the vacuum also contains a gluon condensate.

\section{Pre-QCD: gradient coupling}

In the 1950s, there were many attempts to produce a field theory to describe the interactions of pions and nucleons. The obvious renormalizable interaction between the two objects is the Yukawa coupling to a pseudoscalar: $L_{I}=\bar{N} \gamma_{5} \pi N$

And this is clearly theoretically correct, since it is leading order and it takes all the symmetries into account. But it doesn't match experiment. The interaction that does couples the nucleons to the gradient of the pion field.

$$
g \bar{N} \gamma^{\mu} \partial_{\mu} \pi N
$$

This is the gradient-coupling model. This interaction has a very different dependence on the energy of the pion-it vanishes at zero momentum.

This type of coupling means that a coherent state of low momentum pions barely interacts at all. This is a manifestation of an approximate symmetry, shift symmetry of the pion field. The replacement

$$
\pi \rightarrow \pi+C
$$

Leaves the gradient coupling alone, but not the pseudoscalar coupling.

The modern explanation for the shift symmetry was first proposed by Yoichiro Nambu. The pion field is a Goldstone boson, and the shift symmetry is the lowest order approximation to moving along the flat directions.

\section{Pre-QCD: Goldberger-Treiman relation}

There is a mysterious relationship between the strong interaction coupling of the pions to the nucleons, the coefficient $\mathrm{g}$ in the gradient coupling model, and the axial vector current coefficient of the nucleon which determines the weak decay rate of the neutron. The relation is

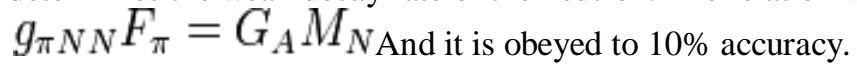


The constant $G_{A}$ is the coefficient that determines the neutron decay rate. It gives the normalization of the weak interaction matrix elements for the nucleon. On the other hand, the pion-nucleon coupling is a phenomenological constant describing the scattering of bound states of quarks and gluons.

The weak interactions are current-current interactions ultimately because they come from a nonabelian gauge theory. The Goldberger Treiman relation suggests that the pions for some reason interact as if they are related to the same symmetry current.

\section{PCAC}

The phenomenon which gives rise to the Goldberger Treiman relation was called the "Partially Conserved Axial Current" hypothesis, or PCAC. Partially conserved is an archaic term for spontaneously broken, and the axial current is now called the chiral symmetry current.

The idea is that the symmetry current which performs axial rotations on the fundamental fields does not preserve the vacuum. This means that the current $\mathbf{J}$ applied to the vacuum produces particles. The particles must be scalars; otherwise the vacuum wouldn't be Lorentz invariant. By index matching, the matrix element is: $J_{\mu}|0\rangle=k_{\mu}|\pi\rangle$, where $k_{\mu}$ is the momentum carried by the created pion. Since the divergence of the axial current operator is zero, we must have

$\partial_{\mu} J^{\mu}|0\rangle=k^{\mu} k_{\mu}|\pi\rangle=m_{\pi}^{2}|\pi\rangle=0$.

Hence the pions are massless, $m_{\pi}^{2}=0$, in accordance with Goldstone's theorem.

Now if the scattering matrix element is considered, we have

$k_{\mu}\left\langle N(p) \mid \pi(k) N\left(p^{\prime}\right)\right\rangle=\left\langle N(p)\left|J_{\mu}\right| N\left(p^{\prime}\right)\right\rangle$.

Up to a momentum factor, which is the gradient in the coupling, it takes the same form as the axial current turning a neutron into a proton in the current-current form of the weak interaction.

$$
\left\langle N\left|J^{\mu}\right| N\right\rangle\left\langle e\left|J_{\mu}\right| \nu\right\rangle
$$

\section{Pre-QCD: soft pion emission}

Extensions of the PCAC ideas allowed Steven Weinberg to calculate the amplitudes for collisions which emit low energy pions from the amplitude for the same process with no pions. The amplitudes are those given by acting with symmetry currents on the external particles of the collision.

These successes established the basic properties of the strong interaction vacuum well before QCD.

\section{Pseudo-Goldstone bosons}

Experimentally it is seen that the masses of the octet of pseudoscalar mesons is very much lighter than the next lightest states; i.e., the octet of vector mesons (such as the rho). The most convincing evidence for SSB of the chiral flavour symmetry of QCD is the appearance of these pseudo-Goldstone bosons. These would have been strictly massless in the chiral limit. There is convincing demonstration that the observed masses are compatible with chiral perturbation theory. The internal consistency of this argument is further checked by lattice QCD computations which allow one to vary the quark mass and check that the variation of the pseudoscalar masses with the quark mass is as required by chiral perturbation theory.

The $\eta^{\prime}$

This pattern of SSB solves one of the mysteries of the quark model where all the pseudoscalar mesons should have been of nearly the same mass. Since $\mathrm{N}_{\mathrm{f}}=3$, there should have been nine of these. However, one (the SU (3) singlet $\eta^{\prime}$ ) has quite a different mass from the SU (3) octet. In the quark model this has no natural explanation - a mystery named the $\boldsymbol{\eta}-\boldsymbol{\eta}^{\prime}$ mass splitting (the $\eta$ is one member of the octet which should have been degenerate in mass with the $\left.\eta^{\prime}\right)$. In QCD one realizes that the $\eta^{\prime}$ is associated with the axial $U$ (1) which is broken through the chiral anomaly. One says therefore, that instantons cause the $\eta-\eta^{\prime}$ mass splitting.

\section{Current algebra and QCD sum rules}

PCAC and current algebra also provide evidence for this pattern of SSB. Direct estimates of the 
chiral condensate also come from such analysis.

Another method of analysis of correlation functions in QCD is through an operator product expansion (OPE). This writes the vacuum expectation value of a non-local operator as a sum over VEVs of local operators, i.e., condensates. The value of the correlation function then dictates the values of the condensates. Analysis of many separate correlation functions gives consistent results for several condensates, including the gluon condensate, the quark condensate, and many mixed and higher order condensates. Here $\mathbf{G}$ refers to the gluon field tensor, $\psi$ to the quark field, and $\mathrm{g}$ to the QCD coupling. These analyses are being refined further through improved sum rule estimates and direct estimates in lattice QCD. They provide the raw data which must be explained by models of the QCD vacuum.

\section{Models of the $Q C D$ vacuum}

A full solution of QCD would automatically give a full description of the vacuum, confinement and the hadron spectrum. ${ }^{[\text {citation needed] }}$ Lattice QCD is making rapid progress towards providing the solution as a systematically improvable numerical computation. However, approximate models of the QCD vacuum remain useful in more restricted domains. The purpose of these models is to make quantitative sense of some set of condensates and hadron properties such as masses and factors. This section is devoted to models. Opposed to these are systematically improvable computational procedures such as large N QCD and lattice QCD, which are described in their own articles.

\section{The Savvidy vacuum, instabilities and structure}

This is a model of the QCD vacuum which at a basic level is a statement that it cannot be the conventional Fock vacuum empty of particles and fields. In 1977, George Savvidy showed that the QCD vacuum with zero field strength is unstable, and decays into a state with a calculable non vanishing value of the field. Since condensates are scalar, it seems like a good first approximation that the vacuum contains some non-zero but homogeneous field which gives rise to these condensates. This would then be a more complicated version of the Higgs mechanism. However, Stanley Mandelstam showed that a homogeneous vacuum field is also unstable. The instability of a homogeneous gluon field was argued by Niels Kjær Nielsenand Poul Olesen in their 1978 paper. These arguments suggest that the scalar condensates are an effective long-distance description of the vacuum, and at short distances, below the QCD scale, the vacuum may have structure.

\section{The dual superconducting model}

In a type II superconductor, electric charges condense into Cooper pairs. As a result magnetic flux is squeezed into tubes. In the dual superconductor picture of the QCD vacuum, chromo magnetic monopoles condense into dual Cooper pairs, causing chromo electric flux to be squeezed into tubes. As a result, confinement and the string picture of hadrons follows. This dual superconductor picture is due to Gerard 't Hooft and Stanley Mandelstam. 't Hooft showed further that an Abelian projection of a nonAbelian gauge theory contains magnetic monopoles.

While the vortices in a type II superconductor are neatly arranged into a hexagonal or occasionally square lattice, as is reviewed in Olesen's 1980 seminar one may expect a much more complicated and possibly dynamical structure in QCD. For example, nonabelian Abrikosov-Nielsen-Olesen vortices may vibrate wildly or be knotted.

\section{String models}

String models of confinement and hadrons have a long history. They were first invented to explain certain aspects of crossing symmetry in the scattering of two mesons. They were also found to be useful in the description of certain properties of the Regge trajectory of the hadrons. These early developments took on a life of their own called the dual resonance model (later renamed string theory). However, even after the development of QCD string models continued to play a role in the physics of strong interactions. These models are called non-fundamental strings or QCD strings, since they should be derived from QCD, as they are, in certain approximations such as the strong coupling limit of lattice QCD.

The model states that the color electric flux between a quark and an antiquark collapses into a string, rather than spreading out into a Coulomb field as the normal electric flux does. This string also obeys a different force law. It behaves as if the string had constant tension, so that separating out the ends (quarks) would give 
a potential energy increasing linearly with the separation. When the energy is higher than that of a meson, the string breaks and the two new ends become a quark-antiquark pair, thus describing the creation of a meson. Thus confinement is incorporated naturally into the model. In the form of the Lund model Monte Carlo program, this picture has had remarkable success in explaining experimental data collected in electronelectron and hadron-hadron collisions.

\section{Bag models}

Strictly, these models are not models of the QCD vacuum, but of physical single particle quantum states - the hadrons. The model consists of putting some version of a quark model in a perturbative vacuum inside a volume of space called a bag. Outside this bag is the real QCD vacuum, whose effect is taken into account through boundary conditions on the quarkwave functions. The hadron spectrum is obtained by solving the Dirac equation for quarks with the bag boundary conditions.

The chiral bag model couples the axial vector current $\bar{\psi} \gamma_{5} \gamma_{\mu} \psi$ of the quarks at the bag boundary to a pionic field outside of the bag. In the most common formulation, the chiral bag model basically replaces the interior of the skyrmion with the bag of quarks. Very curiously, most physical properties of the nucleon become mostly insensitive to the bag radius. Prototypically, the baryon number of the chiral bag remains an integer, independent of bag radius: the exterior baryon number is identified with the topological winding number density of the Skyrme soliton, while the interior baryon number consists of the valence quarks (totaling to one) plus the spectral asymmetry of the quark eigenstates in the bag. The spectral asymmetry is just the vacuum expectation value $\left\langle\bar{\psi} \gamma_{0} \psi\right\rangle$ summed over all of the quark eigenstates in the bag. Other values, such as the total mass and the axial coupling constant $\boldsymbol{g}_{\boldsymbol{A}}$, are not precisely invariant like the baryon number, but are mostly insensitive to the bag radius, as long as the bag radius is kept below the nucleon diameter. Because the quarks are treated as free quarks inside the bag, the radius-independence in a sense validates the idea of asymptotic freedom

\section{Instanton ensemble}

Another view states that BPST-like instantons play an important role in the vacuum structure of QCD. These instantons were discovered in 1975 by Belavin, Polyakov, Schwartz andTyupkin ${ }^{[}$as topologically stable solutions to the Yang-Mills field equations. They represent tunneling transitions from one vacuum state to another. These instantons are indeed found in lattice calculations. The first computations performed with instantons used the dilute gas approximation. The results obtained did not solve the infrared problem of QCD, making many physicists turn away from Instanton physics. Later, though, an Instanton liquid model was proposed, turning out to be more promising an approach

The dilute Instanton gas model departs from the supposition that the QCD vacuum consists of a gas of BPST-like instantons. Although only the solutions with one or few instantons (or anti-instantons) are known exactly, a dilute gas of instantons and anti-instantons can be approximated by considering a superposition of one-Instanton solutions at great distances from one another. 't Hooft calculated the effective action for such an ensemble, ${ }^{]}$and he found an infrared divergence for big instantons, meaning that an infinite amount of infinitely big instantons would populate the vacuum.

Later, an Instanton liquid model was studied. This model starts from the assumption that an ensemble of instantons cannot be described by a mere sum of separate instantons. Various models have been proposed, introducing interactions between instantons or using vibrational methods (like the "valley approximation") endeavoring to approximate the exact multi-Instanton solution as closely as possible. Many phenomenological successes have been reached. Whether an Instanton liquid can explain confinement in $3+1$ dimensional QCD is not known, but many physicists think that it is unlikely.

\section{Center vortex picture}

A more recent picture of the QCD vacuum is one in which center vortices play an important role. These vortices are topological defects carrying a center element as charge. These vortices are usually studied using lattice simulations, and it has been found that the behavior of the vortices is closely linked with the confinement-deconfinement phase transition: in the confining phase vortices percolate and fill the space- 
time volume, in the deconfining phase they are much suppressed. Also it has been shown that the string tension vanished upon removal of center vortices from the simulations, hinting at an important role for center vortices. The QCD vacuum is the vacuum state of quantum chromodynamics (QCD). It is an example of a non-perturbative vacuum state, characterized by many non-vanishing condensates such as the gluon condensate or the quark condensate. These condensates characterize the normal phase or the confined phase of quark matter. $Q C D$ in the non-perturbative regime: confinement. The equations of $\mathrm{QCD}$ remain unsolved at energy scales relevant for describing atomic nuclei. How does QCD give rise to the physics of nuclei and nuclear constituents?

\section{VACCUM ENERGY RESPONSIBLE FOR BIG BANG AND FORMATION OF BASIC FORCES:}

General relativity theory combined the concepts of space and time in essence creating a substance that one could qualify as aether. Aether became a substance that could be warped (gravity) and would bend the path that light travels. He put forth the idea that gravity and acceleration was both a product of the same phenomena.

Being acquainted with Lorentz' time dilation formulae, Einstein were able to show that spacetime was basically a field in which time flowed at a certain rate. Any acceleration and motion through this field caused the flow rate of time for the said object to run at a slower rate, and gravity was equated with being acceleration. For instance we are being accelerated towards the center of the earth by about 9.8 meters per sec. per sec.

Atomic clocks have shown that this concept is true. They found that clocks run at a slower rate closer to the earth than clocks that are further away. We feel this time flow rate slowdown as an acceleration, or gravity. Similarly, when we accelerate we feel it in the same way as we do gravity, and it can affect the flow rate of time in the same fashion. Atomic clocks have shown these concepts to be fairly correct as well. Any increase in motion in regards to a more stationary frame of reference causes a slowdown in the flow rate of time for the frame of reference in motion.

If we could accelerate objects, (even sub atomic particles) which we do, we would find that their flow rate of time is much slower (e)than if they were stationary because it takes a much longer period of time for certain particles to break down, when they are moving at speeds nearing the speed of $c$, than not.

This means that we, and all objects of mass, must contend with an energy field which alters the flow rate of time, when this field is influenced. We might be able to even say that this energy field is the flow rate of time. Spacetime then can be likened to an energy field. It even affects all electromagnetic energy as well when the path that it travels is warped via gravity.

Einstein also at one time pictured the universe as static, or basically unchanging. However because of gravity, he knew that this state could not continue. If the universe was under the influence of gravity alone, all in the universe would eventually collapse. The universe would eventually pull itself together into one blob of matter. To offset this unpalatable outcome, he put in a fudge factor that counteracted gravity and held the universe in place.Later it was found that the universe is expanding. Galaxies in general were flying away from each other, so there was no need for this fudge factor to have been put into his description of the universe. The momentum of their flying away from each other solved the problem of a collapsing universe. Or so it was thought.

Since then we have discovered that it is not a momentum at all that is causing the universe to expand, because if it were then gravity would at least be slowing the expansion rate down. Maybe it did so once billions of years ago, but now we've learned that things are accelerating away from each other. That means that Einstein's original idea of energy in opposition to gravity and holding things apart was correct. In fact the number value for his cosmological constant is even greater than he first postulated, because it has overcome the force of gravity and is accelerating, not merely holding it in equilibrium. What might this cosmological constant be? Space-time, ether, or a cosmological constant energy field? Particle physicists have another name for such an all pervasive energy field. 
Particle physicists call such a field, the false vacuum. It is space that contains energy. Is it possible to believe that it is this energy that caused(eb) the Big Bang, and is what is responsible for the increased expansion of the universe? If space is not empty as some might suppose, but it is filled with this false vacuum, might it not be the very same thing as the spacetime that Einstein had also postulated?

This false vacuums energy, the energy of the cosmological constant, and Einstein's spacetime, are really one and the same.

How does this false vacuum make the universe expand?

Here is how one cosmology book puts it that discusses Einstein's greatest blunder:

"We must consider the possibility that space itself possesses energy. As the false vacuum expands, each cubic centimeter continues to have the same amount of energy as before. The decrease in the amount of energy per cubic centimeter that anyone would reasonably expect from the expansion simply does not occur. When more space appears, the total amount of energy in all space rises in direct proportion. Thus, as the universe expands it creates not only new space but also new energy."

With increasing space comes increasing energy as well between the galaxies. It made the statement that the universe would slow down in its expansion rate at the beginning due to gravity, then it would reach a sort of coasting or equilibrium point for a time, (after which it would begin to slowdown again.)

But if you took his previous statement about: "...with increasing space comes increasing energy as well." That alone would tell you that after some coasting point in the expansion rate, that eventually if the expansion rate continued, the universe's expansion rate should then start to increase, as the distances and thus energies also increased between the galaxies and gravity's strength decreased. This is dark energy Einstein's spacetime, The false vacuum energy, dark energy, the cosmological constant energy, all could be part of one and the same thing, the aether. ?

So as the universe expands, space-time, the vacuum energy, or the ether does too. In fact it is this energy field ether that drives the expansion of the universe. Spacetime expands the universe.

\section{Work Suggested/Done: VACUUM ENERGY(VE) PORTFOLIO: ASSUMPTIONS:}

VE IS classified into three categories;

1) Category 1 representative OF VE vis-à-vis Quantum Field (QF) in the corresponding category

2) Category 2 (second interval ) comprising of VE corresponding to QF in the category 2

3) Category 3 constituting $\mathrm{VE}$ vis a vis $\mathrm{QF}$ in category 3

a) In this connection, it is to be noted that there is no sacrosanct time scale as far as the above pattern of classification is concerned. Any operationally feasible scale with an eye on the QF-VE system would be in the fitness of things. For category 3. "Over and above" nomenclature could be used to encompass a wider range of consumption due to cellular respiration. Similarly, a "less than" scale for category 1 can be used.

b) The speed of growth of VE in category 1 is proportional to the total amount of VF of category 2. In essence the accentuation coefficient in the model is representative of the constant of proportionality between VF under category 1 and category 2 this assumptions is made to foreclose the necessity of addition of one more variable, that would render the systemic equations unsolvable

c) The dissipation in all the three categories is attributable to the following two phenomenon :

1) Aging phenomenon: The aging process leads to transference of the balance of VE (like it happens in the case of age of the matter) to the next category

Depletion phenomenon: VE dissipation that occurs due to continuous process of transformation of one to another and holistically maintenance of energy conservation and conservation universistically, In this connection attention is drawn to the fact that in a bank individual debits and credits are tallied and globally as a bank, the totalistic debits and credits are tallied. The transformation of one energy in to another itself has an analogy in the individual debit and credit syllogism while the holistic writing of the General Ledger corresponds to the General theory of the transformation of one energy in to another each such transformation 
in the universe recorded as it is in the General Ledger in the Bank.

\section{NOTATION :}

$G_{24}:$ Quantum of VF in category 1

$G_{25}:$ Quantum of VF in category2

$\mathrm{G}_{26:}$ Quantum of VFin category 3

$\left(a_{24}\right)^{(4)},\left(\mathrm{a}_{25}\right)^{(4)},\left(\mathrm{a}_{26}\right)^{(4)}:$ Accentuation coefficients

$\left(a_{24}^{\prime}\right)^{(4)},\left(a_{25}^{\prime}\right)^{(4)},\left(a_{26}^{\prime}\right)^{(4)}$ : Dissipation coefficients

FORMULATION OF THE SYSTEM :

In the light of the assumptions stated in the foregoing, we infer the following:-

(a)The growth speed in category 1 is the sum of a accentuation term $\left(a_{24}\right)^{(4)} \mathrm{G}_{25}$ and a dissipation term - $\left(\mathrm{a}_{24}^{\prime}\right)^{(4)} G_{24}$, the amount of dissipation taken to be proportional to that in category 2

(b) The growth speed in category 2 is the sum of two parts $\left(a_{25}\right)^{(4)} G_{24}$ and $-\left(a_{25}^{\prime}\right)^{(4)} G_{25}$ the inflow from the category 1 dependent on the total amount standing in that category.

(c)The growth speed in category 3 is equivalent to $\left(a_{26}\right)^{(4)} G_{25}$ and $-\left(\mathrm{a}_{26}^{\prime}\right)^{(4)} \mathrm{G}_{26}$ dissipation ascribed only to depletion phenomenon.

Model makes allowance for the new VF and QF entering and disgorged from the system under consideration

\section{GOVERNING EQUATIONS:}

The differential equations governing the above system can be written in the following form

$$
\begin{aligned}
& \frac{d G_{24}}{d t}=\left(a_{24}\right)^{(4)} G_{25}-\left(a_{24}^{\prime}\right)^{(4)} G_{24} \\
& \frac{d G_{25}}{d t}=\left(a_{25}\right)^{(4)} G_{24}-\left(a_{25}^{\prime}\right)^{(4)} G_{25} \\
& \frac{d G_{26}}{d t}=\left(a_{26}\right)^{(4)} G_{25}-\left(a_{26}^{\prime}\right)^{(4)} G_{26} \\
& \left(a_{i}\right)^{(4)}>0 \quad, \quad i=24,25,26 \\
& \left(a_{i}^{\prime}\right)^{(4)}>0 \quad, \quad i=24,25,26 \\
& \left(a_{25}\right)^{(4)}<\left(a_{24}^{\prime}\right)^{(4)} \\
& \left(a_{26}\right)^{(4)}<\left(a_{25}^{\prime}\right)^{(4)}
\end{aligned}
$$

We can rewrite equation 4, 2 and 3 in the following form

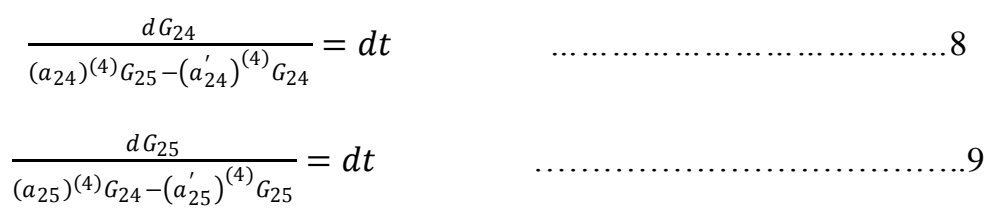


Or we write a single equation as

$$
\frac{d G_{24}}{\left(a_{24}\right)^{(4)} G_{25}-\left(a_{24}^{\prime}\right)^{(4)} G_{24}}=\frac{d G_{25}}{\left(a_{25}\right)^{(4)} G_{24}-\left(a_{25}^{\prime}\right)^{(4)} G_{25}}=\frac{d G_{26}}{\left(a_{26}\right)^{(4)} G_{25}-\left(a_{26}^{\prime}\right)^{(4)} G_{26}}=d t
$$

The equality of the ratios in equation (10) remains unchanged in the event of multiplication of numerator and denominator by a constant factor.

For constant multiples $\alpha, \beta, \gamma$ all positive we can write equation (10) as

$$
\frac{\alpha d G_{24}}{\alpha\left(\left(a_{24}\right)^{(4)} G_{25}-\left(a_{24}^{\prime}\right)^{(4)} G_{24}\right)}=\frac{\beta d G_{25}}{\beta\left(\left(a_{25}\right)^{(4)} G_{24}-\left(a_{25}^{\prime}\right)^{(4)} G_{25}\right)}=\frac{\gamma d G_{26}}{\gamma\left(\left(a_{26}\right)^{(4)} G_{25}-\left(a_{26}^{\prime}\right)^{(4)} G_{26}\right)}=d t
$$

The general solution system can be written in the form

$$
\alpha_{i} G_{i}+\beta_{i} G_{i}+\gamma_{i} G_{i}=C_{i} e_{i}^{\lambda_{i} t} \text { Where } i=24,25,26 \text { and } C_{24}, C_{25}, C_{26} \text { are arbitrary constant coefficients. }
$$

\section{STABILITY ANALYSIS :}

Supposing $G_{i}(0)=G_{i}^{0}(0)>0$, and denoting by $\lambda_{i}$ the characteristic roots of the system, it easily results that

(1). If $\left(a_{24}^{\prime}\right)^{(4)}\left(a_{25}^{\prime}\right)^{(4)}-\left(a_{24}\right)^{(4)}\left(a_{25}\right)^{(4)}>0$ all the components of the solution, i.e. all the three parts of the system tend to zero, and the solution is stable with respect to the initial data.

2. If $\left(a_{24}^{\prime}\right)^{(4)}\left(a_{25}^{\prime}\right)^{(4)}-\left(a_{24}\right)^{(4)}\left(a_{25}\right)^{(4)}<0 \quad$ and $\left(\lambda_{25}+\left(a_{24}^{\prime}\right)^{(4)}\right) G_{24}^{0}-\left(a_{24}\right)^{(4)} G_{25}^{0} \neq 0,\left(\lambda_{25}<0\right)$, the first two components of the solution tend to infinity as $t \rightarrow \infty$, and $G_{26} \rightarrow 0$, ie. The category 1 and category 2 parts grows to infinity, whereas the third part category 3 tends to zero.

3. If $\left(a_{24}^{\prime}\right)^{(4)}\left(a_{25}^{\prime}\right)^{(4)}-\left(a_{24}\right)^{(4)}\left(a_{25}\right)^{(4)}<0$ and $\left(\lambda_{25}+\left(a_{24}^{\prime}\right)^{(4)}\right) G_{24}^{0}-\left(a_{24}\right)^{(4)} G_{25}^{0}=0$ Then all the three parts tend to zero, but the solution is not stable i.e. at a small variation of the initial values of $\mathrm{G}_{i}$, the corresponding solution tends to infinity.

\section{LSST PROJECT-TOTAL ENERGY DENSITY OF THE UNIVERSE-AN ESSENTIAL PREREQUISITE FOR CLASSFIFICATION OF THE QUANTUM FIELD;IS DARK ENERGY VACUUM ENERGY?}

(1)Dark energy is a mysterious force that is accelerating the expansion of the universe. Expansion of the universe is due to dark energy.(2) The expansion has slowed the clustering of dark matter, one of the universe's main building blocks. If we could measure the precise history of the Hubble expansion, and chart the development of mass structure, we could test theories of the physics of dark energy.

The LSST program me concentrates on and will enable scientists to study the dark energy in four different and complementary ways:

The telescope will image dark matter over cosmic time, via a "gravitational mirage." All the galaxies behind a clump of dark matter are deflected to a new place in the sky, causing their images to be distorted. This is effectively 3-D mass tomography of the universe.

Galaxies clump in a non-random way, guided by the natural scale that was imprinted in the fireball of the Big Bang. This angular scale will be measured over cosmic time by LSST, yielding valuable information on the changing Hubble expansion.

The numbers of huge clusters of dark matter are a diagnostic of the underlying cosmology. By charting the numbers of these (via their gravitational mirage) over cosmic time, LSST will place another sensitive constraint on the physics of dark energy. 
Finally, a million supernovae will be monitored by LSST, giving yet another complementary view of the history of the Hubble expansion.

\section{Something Is Ripping the Universe Apart}

Recently the composition of the universe has become even more puzzling: observations imply an acceleration of the universe's expansion over the past few billion years. In order to explain such acceleration, we need "dark energy" with large negative pressure to generate a repulsive gravitational force. The evidence comes from studies of the total energy density of the universe and from supernova observations. Precision measurements of the cosmic microwave background have shown that the total energy density of the universe is very near the critical density needed to make the universe flat (i.e. the curvature of space-time, defined in General Relativity, goes to zero on large scales). Since energy is equivalent to mass (Special Relativity: $\mathrm{E}=\mathrm{mc} 2$ ), this is usually expressed in terms of a critical mass density needed to make the universe flat. Ordinary matter such as stars, dust, and gas account for only $5 \%$ of the necessary mass density. Observations have shown that dark matter cannot account for more than $\sim 25 \%$ of the critical mass density. Both the microwave background and supernova observations suggest that dark energy should make up $\sim 70 \%$ of the critical energy density. When added to the mass-energy of matter, the total energy density is consistent with what is needed to make the universe flat.

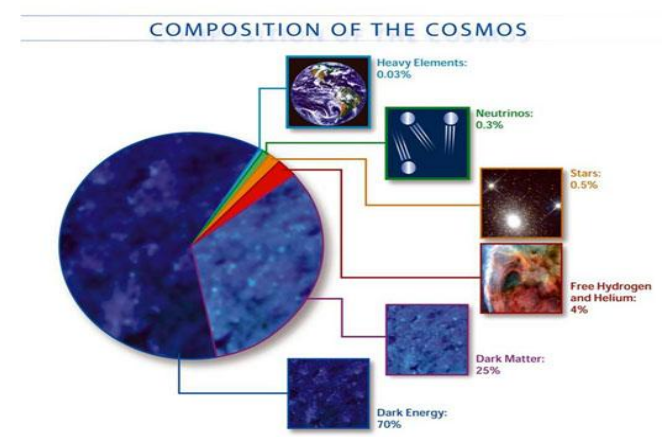

Figure reproduced from the Dark Energy Home Page

Over the expansion history of our universe, densities have fallen by factors of trillions. Why is the dark energy density today within a factor of three of that of dark matter, whereas it evolves very differently with time? Moreover, the dark matter density is only a factor of five larger than that of ordinary matter. Understanding this may lead to advances in fundamental physics. It is possible that what we call dark matter and dark energy arises from some unknown aspect of gravity. Thus, the highest energies and the universe on the largest scales are connected. Today the worlds of particle physics and cosmology are coming together in a transformed world view. Now, even the notion that the galaxies and stars comprise most of our universe has been abandoned. Emerging is a universe largely governed by dark matter and an even stranger dominance of a smoothly distributed and pervasive dark energy.

\section{Dark Energy and the Fate of the Universe}

Cosmologists understand almost nothing about dark energy even though it appears to comprise about 70 percent of the universe. They are desperately seeking to uncover its fundamental properties: its strength, its permanence, and any variation with direction. The evolution of the universe is governed by the amount of dark matter and dark energy. The densities of dark matter and dark energy scale differently with cosmic scale as the universe expands. This evolution in cosmic scale is schematically shown in the figure below for several cosmologies. In a universe with a high density of dark matter, the Hubble expansion continues to decelerate due to the gravitation attraction of the dark matter filling the universe, ending in a big crunch. In a universe with a lower critical density of dark matter, the expansion coasts. In a universe with dark energy as well as dark matter, the initial deceleration is reversed at late times by the increasing dominance of the 
dark energy.

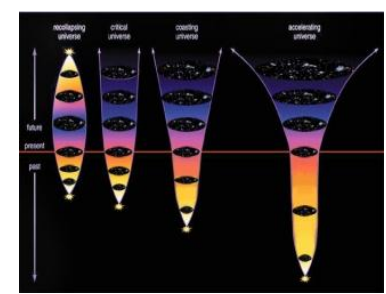

If the hypothetical dark energy continues to dominate the universe's energy balance, then the current expansion of space will continue to accelerate, exponentially. Structures which are not already gravitationally bound will ultimately fly apart. The Earth and the Milky Way would remain undisturbed while the rest of the universe appears to run away from us. The nature of dark energy is currently a matter of speculation. Some believe that dark energy might be "vacuum energy", represented by the "cosmological constant" $(\Lambda)$ in general relativity, a constant uniform density of dark energy throughout all of space that is independent of time or the universe's expansion. This notion was introduced by Einstein, and is consistent with our limited observations to date. Alternatively, dark energy might vary with cosmic time. Only new kinds of observations can settle the issue.

\section{PROBING THE NATURE OF DARK ENERGY}

What kind of universe do we live in? To test theories of dark energy we would like to measure the way the expansion of our universe changes with cosmic time. For a universe with a given mass density, the time history of the expansion encodes information on the amount and nature of dark energy. Dark energy affects two things: distances and the growth of mass structure. Measuring how dark matter structures and ratios of distances grow with cosmic time -- via LSST weak gravitational lensing observations -- will provide clues to the nature of dark energy. A key strength of the LSST is its ability to image huge volumes of the universe. Such a probe will be a natural part of the all-sky imaging survey. Billions of distant galaxies will have their shapes and colors measured. Sufficient color data will be obtained for an estimate of the distance to each galaxy. This will enable a unique probe of the physical nature of the dark energy that appears to fill the universe.

If, due to this dark energy, the expansion of the universe has been accelerating the development of mass structures via ordinary gravitational in fall will be impeded. The time development of mass concentrations is sensitive to the physical nature of the dark energy itself: the so-called equation of state (pressure divided by energy density). In its deep wide-angle survey the LSST will be able to pin down the equation of state of dark energy to better than a few percent, at high confidence. Due to its wide coverage of the sky, LSST is uniquely capable of detecting any variation in the dark energy with direction. In turn, this will tell us something about physics at the earliest moments of our universe, setting the course for its future evolution. The world of quantum gravity at a fraction of a second after the big bang, when the universe was so hot and dense that even protons and neutrons were broken up into a hot soup of quarks, connects to the world as we now see it - a vast expanding cosmos extending out 14 billion light-years. Dark energy and dark matter are relics of the first moments when unfamiliar physics of quantum gravity ruled. A route to understanding dark matter and probing the nature of dark energy is to measure cosmic shear over the last half of the age of the universe - at a time when dark energy apparently had its greatest influence. LSST does this in several independent ways. These probes of the nature of dark energy by LSST are complimentary to those of space missions measuring the cosmic microwave background and very distant supernovae. Indeed, since we understand so little about dark energy, it is prudent to pursue all these lines of investigation. 


\section{Splitting malleable fabric of Time from Space-Quantum Gravity and Vacuum Energy}

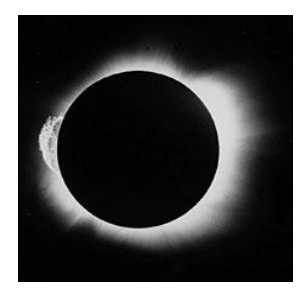

A solar eclipse confirmed gravitational lensing and Einstein's concept of spacetime. But a new quantum gravity theory now generating excitement separates time and space. Image: Photo Researchers, Inc.

\section{Supplemental Material}

\section{Sidebar Splitting Time from Space The Evidence}

It seems that unzipping the fabric of spacetime and harking back to 19th-century notions of time could lead to a theory of quantum gravity.Physicists have struggled to marry quantum mechanics with gravity for decades. In contrast, the other forces of nature have obediently fallen into line. For instance, the electromagnetic force can be described quantum-mechanically by the motion of photons. Try and work out the gravitational force between two objects in terms of a quantum graviton, however, and you quickly run into trouble - the answer to every calculation is infinity. Some think it is about a matter of time. More specifically, the problem is the way that time is tied up with space in Einstein's theory of gravity: general relativity. Einstein famously overturned the Newtonian notion that time is absolute - steadily ticking away in the background. Instead he argued that time is another dimension, woven together with space to form a malleable fabric that is distorted by matter. The snag is that in quantum mechanics, time retains its Newtonian aloofness, providing the stage against which matter dances but never being affected by its presence. These two conceptions of time don't gel.

The solution many quantum gravity proponents and precursors is to snip threads that bind time to space at very high energies, such as those found in the early universe where quantum gravity rules. "I'm going back to Newton's idea that time and space is not equivalent," HoYava says. At low energies, general relativity emerges from this underlying framework, and the fabric of spacetime restitches. Many liken this emergence to the way some exotic substances change phase. For instance, at low temperatures liquid helium's properties change dramatically, becoming a "super fluid" that can overcome friction. In fact, Ho Yava has co-opted the mathematics of exotic phase transitions to build his theory of gravity. So far it seems to be working: the infinities that plague other theories of quantum gravity have been tamed, and the theory disgorges well-behaved graviton. It also seems to match with computer simulations of quantum gravity. In particular, physicists have been checking if the model correctly describes the universe we see today. General relativity scored a knockout blow when Einstein predicted the motion of Mercury with greater accuracy than Newton's theory of gravity could.

Cosmic conundrums such as the singularity of the big bang, where the laws of physics break down, can be explained by the theory of gravity. If HoYava gravity is true, argues cosmologist Robert Brandenberger of McGill University in a paper published in the August Physical Review D, then the universe didn't bang — it bounced. "A universe filled with matter will contract down to a small—but finite — size and then bounce out again, giving us the expanding cosmos we see today," he says. Brandenberger calculations show that ripples produced by the bounce match those already detected by satellites measuring the cosmic microwave background, and he is now looking for signatures that could distinguish the bounce from the big bang scenario. 
HoYava gravity may also create the "illusion of dark matter," says cosmologist Shinji Mukohyama of Tokyo University. In the September Physical Review D, he explains that in certain circumstances HoYava's graviton fluctuates as it interacts with normal matter, making gravity pull (INCREASE IN THE GRAVITATIONAL FORCE)a bit more strongly than expected in general relativity. The effect could make galaxies appear to contain more matter than can be seen. If that's not enough, cosmologist Mu-In Park of Chonbuk National University in South Korea believes that HoYava gravity may also be behind the accelerated expansion of the universe, currently attributed to a mysterious dark energy. One of the leading explanations for its origin is that empty space contains some intrinsic energy that pushes (expands) the universe outward. This intrinsic energy cannot be accounted for by general relativity but pops naturally out of the equations of HoYava gravity, according to Park.

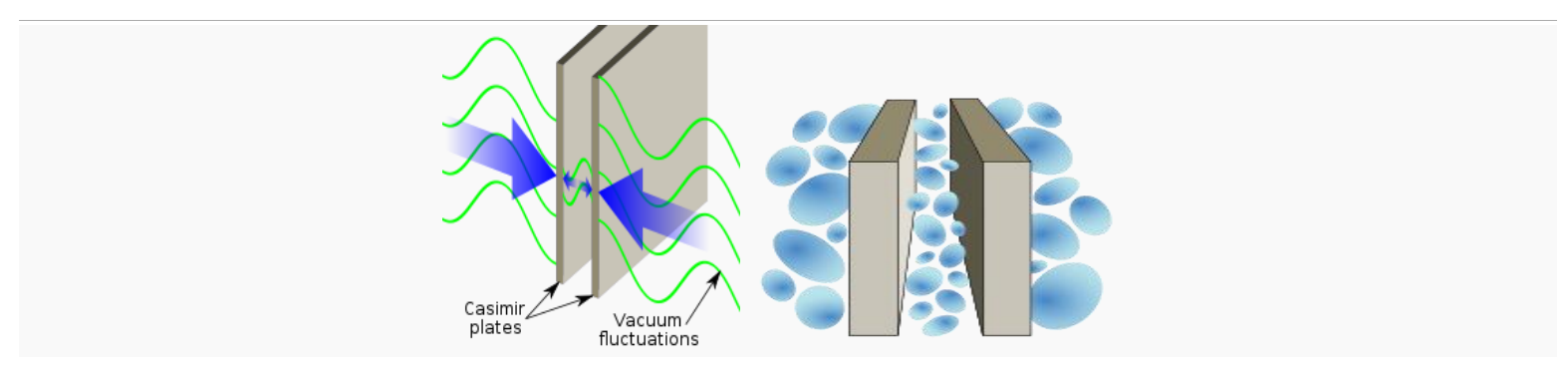

Casimir effect and vacuum energy fluctuations: $\quad$ Casimir forces on parallel plates

In quantum field theory, the Casimir effect and the Casimir-Polder force are physical forces arising from a quantized field. The typical example is of two uncharged metallic plates in a vacuum, like capacitors placed a few micrometers apart, without any external electromagnetic field. In a classical description, the lack of an external field also means that there is no field between the plates, and no force would be measured between them. When this field is instead studied using the QED vacuum of quantum electrodynamics, it is seen that the plates do affect the virtual photons which constitute the field, and generate a net force- either an attraction or a repulsion depending on the specific arrangement of the two plates. Although the Casimir effect can be expressed in terms of virtual particles interacting with the objects, it is best described and more easily calculated in terms of the zero-point energy of a quantized field in the intervening space between the objects. This force has been measured, and is a striking example of an effect purely due to second quantization However, the treatment of boundary conditions in these calculations has led to some controversy. In fact "Casimir's original goal was to compute the van der Waals force between polarizable molecules" of the metallic plates. Thus it can be interpreted without any reference to the zero-point energy (vacuum energy) or virtual particles of quantum fields.

Because the strength of the force falls off rapidly with distance, it is only measurable when the distance between the objects is extremely small. On a submicron scale, this force becomes so strong that it becomes the dominant force between uncharged conductors. In fact, at separations of $10 \mathrm{~nm}$-about 100 times the typical size of an atom - the Casimir effect produces the equivalent of 1 atmosphere of pressure $(101.325$ $\mathrm{kPa}$ ), the precise value depending on surface geometry and other factors. In modern theoretical physics, the Casimir effect plays an important role in the chiral bag model of the nucleon; and in applied physics, it is significant in some aspects of emerging micro technologies and nanotechnologies

The Casimir effect can be understood by the idea that the presence of conducting metals and dielectrics alters the vacuum expectation value of the energy of the second quantized electromagnetic Since the value of this energy depends on the shapes and positions of the conductors and dielectrics, the Casimir effect makes itself manifest as a force between such objects.

\section{VACUUM ENERGY}

The causes of the Casimir effect are described by quantum field theory, which states that all of the various www.iosrjournals.org 
fundamental fields, such as the electromagnetic, must be quantized at each and every point in space. In a simplified view, a "field" in physics may be envisioned as if space were filled with interconnected vibrating balls and springs, and the strength of the field can be visualized as the displacement of a ball from its rest position. Vibrations in this field propagate and are governed by the appropriate wave equation for the particular field in question. The second quantization of quantum field theory requires that each such ballspring combination be quantized, that is, that the strength of the field be quantized at each point in space. At the most basic level, the field at each point in space is a simple harmonic oscillator, and its quantization places a quantum harmonic oscillator at each point. Excitations of the field correspond to the elementary particles of particle physics. However, even the vacuum has a vastly complex structure, so all calculations of quantum field theory must be made in relation to this model of the vacuum.

The vacuum has, implicitly, all of the properties that a particle may have: spin, or polarization in the case of light, energy, and so on. On average, most of these properties cancel out: the vacuum is, after all, "empty" in this sense. One important exception is the vacuum energy or the vacuum expectation value of the energy. The quantization of a simple harmonic oscillator states that the lowest possible energy orzeropoint energy that such an oscillator may have is

\section{$E=\frac{1}{2} \hbar \omega$}

Summing over all possible oscillators at all points in space gives an infinite quantity. To remove this infinity, one may argue that only differences in energy are physically measurable; this argument is the underpinning of the theory of renormalization. In all practical calculations, this is how the infinity is always handled. In a deeper sense, however, renormalization is unsatisfying, and the removal of this infinity presents a challenge in the search for a Theory of Everything. Currently there is no compelling explanation for how this infinity should be treated as essentially zero; a non-zero value is essentially the cosmological constant and any large value causes trouble in cosmology.

Alternatively, a 2005 paper by Robert Jaffe of MIT states that "Casimir effects can be formulated and Casimir forces can be computed without reference to zero point energies. They are relativistic, quantum forces between charges and currents. The Casimir force (per unit area) between parallel plates vanishes as alpha, the fine structure constant, goes to zero, and the standard result, which appears to be independent of alpha, corresponds to the alpha $\rightarrow$ infinity limit," and that "The Casimir force is simply the (relativistic, retarded) van der Waals force between the metal plates.

Casimir's observation was that the second-quantized quantum electromagnetic field, in the presence of bulk bodies such as metals or dielectrics, must obey the same boundary conditions that the classical electromagnetic field must obey. In particular, this affects the calculation of the vacuum energy in the presence of a conductor or dielectric.

Consider, for example, the calculation of the vacuum expectation value of the electromagnetic field inside a metal cavity, such as, for example, a radar cavity or a microwave waveguide. In this case, the correct way to find the zero point energy of the field is to sum the energies of the standing waves of the cavity. To each and every possible standing wave corresponds energy; say the energy of the $n$th standing wave is $E_{n}$. The vacuum expectation value of the energy of the electromagnetic field in the cavity is then $\langle E\rangle=\frac{1}{2} \sum_{n} E_{n}$

With the sum running over all possible values of $n$ enumerating the standing waves. The factor of $1 / 2$ corresponds to the fact that the zero-point energies are being summed (it is the same $1 / 2$ as appears in the equation $E=\hbar \omega / 2$ ). Written in this way, this sum is clearly divergent; however, it can be used to 
create finite expressions.

In particular, one may ask how the zero point energy depends on the shape $s$ of the cavity. Each energy level $E_{n} \underline{\text { depends }}$ on the shape, and so one should write $E_{n}(s)$ for the energy level, and $\langle E(s)\rangle$ for the vacuum expectation value. At this point comes an important observation: the force at point $p$ on the wall of the cavity is equal to the change in the vacuum energy if the shape $s$ of the wall is perturbed a little bit, say by $\delta s$, at point $p$. That is, one has

$F(p)=-\left.\frac{\delta\langle E(s)\rangle}{\delta s}\right|_{p}$

This value is finite in many practical calculations. Attraction between the plates can be easily understood by focusing on the 1-dimensional situation. Suppose that a moveable conductive plate is positioned at a short distance $a$ from one of two widely separated plates (distance $L$ apart). With $a<<$, the states within the slot of width $a$ are highly constrained so that the energy $E$ of any one mode is widely separated from that of the next. This is not the case in open region $L$, where there is a large number (about $L / a$ ) of states with energy evenly spaced between $E$ and the next mode in the narrow slot---in other words, all slightly larger than $E$. Now on shortening $a$ by $\mathrm{d} a(<0)$, the mode in the slot shrinks in wavelength and therefore increases in energy proportional to $-\mathrm{d} a / a$, whereas all the outside $L / a$ states lengthen and correspondingly lower energy proportional to $\mathrm{d} a / L$ (note the denominator). The net change is slightly negative, because all the $L / a$ modes' energies are slightly larger than the single mode in the slot.

\section{Zeta-regularization-Vacuum energy as the sum of all excitation modes:}

In the original calculation done by Casimir, he considered the space between a pair of conducting metal plates at distance $a$ apart. In this case, the standing waves are particularly easy to calculate, since the transverse component of the electric field and the normal component of the magnetic field must vanish on the surface of a conductor. Assuming the parallel plates lie in the xy-plane, the standing waves are

$$
\psi_{n}(x, y, z ; t)=e^{-i \omega_{n} t} e^{i k_{x} x+i k_{y} y} \sin \left(k_{n} z\right)
$$

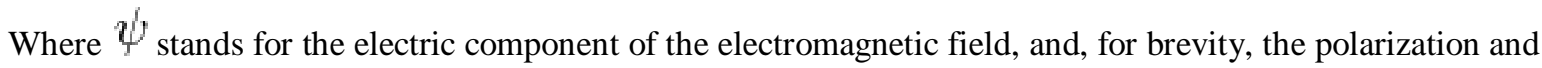
the magnetic components are ignored here. Here, $k_{x}$ and $k_{y}$ are the wave vectors in directions parallel to the plates, and

$k_{n}=\frac{n \pi}{a}$

is the wave-vector perpendicular to the plates. Here, $n$ is an integer, resulting from the requirement that $\psi$ vanish on the metal plates. The energy of this wave is

$\omega_{n}=c \sqrt{k_{x}{ }^{2}+k_{y}{ }^{2}+\frac{n^{2} \pi^{2}}{a^{2}}}$

where $c$ is the speed of light. The vacuum energy is then the sum over all possible excitation modes

$$
\langle E\rangle=\frac{\hbar}{2} \cdot 2 \int \frac{A d k_{x} d k_{y}}{(2 \pi)^{2}} \sum_{n=1}^{\infty} \omega_{n}
$$

Where $A$ is the area of the metal plates, and a factor of 2 is introduced for the two possible polarizations of the wave. This expression is clearly infinite, and to proceed with the calculation, it is convenient to introduce a regulator the regulator will serve(EB) to make the expression finite, and in the end will be removed. The zeta-regulated version of the energy per unit-area of the plate is

$$
\frac{\langle E(s)\rangle}{A}=\hbar \int \frac{d k_{x} d k_{y}}{(2 \pi)^{2}} \sum_{n=1}^{\infty} \omega_{n}\left|\omega_{n}\right|^{-s}
$$

In the end, the limit $s \rightarrow 0$ is to be taken. Here $s$ is just a complex number, not to be confused with the www.iosrjournals.org 
shape discussed previously. This integral/sum is finite for $s$ real and larger than 3 . The sum has a pole at $s=3$, but may be analytically continued to $s=0$, where the expression is finite. The above expression is easily simplified to:

$$
\frac{\langle E(s)\rangle}{A}=\frac{\hbar c^{1-s}}{4 \pi^{2}} \sum_{n} \int_{0}^{\infty} 2 \pi q d q\left|q^{2}+\frac{\pi^{2} n^{2}}{a^{2}}\right|^{(1-s) / 2}
$$

Where polar coordinates $q^{2}=k_{x}^{2}+k_{y}^{2}$ were introduced to turn the double integral into a single integral. The $q$ in front is the Jacobin, and the $2 \pi$ comes from the angular integration. The integral is easily performed and converges if $\operatorname{Re}[s]>3$, resulting in

$$
\frac{\langle E(s)\rangle}{A}=-\frac{\hbar c^{1-s} \pi^{2-s}}{2 a^{3-s}} \frac{1}{3-s} \sum_{n}|n|^{3-s}
$$

The sum clearly diverges at $s$ in the neighborhood of zero, but if the damping of large-frequency excitations corresponding to analytic continuation of the Riemann zeta function to $s=0$ is assumed to make sense physically in some way,

\section{WORM HOLE AND VACUUM ENERGY}

Exotic matter with negative energy density may be required to stabilize a wormhole. Morris, Thorne and Yurtsever pointed out that the quantum mechanics of the Casimir effect can be used to produce a locally mass-negative region of space-time and suggested that negative effect could be used to stabilize a wormhole to allow faster than light travel.

In physics, a wormhole is a hypothetical topological feature of spacetime that would be, fundamentally, a "shortcut" through spacetime. For a simple visual explanation of a wormhole, consider spacetime visualized as a two-dimensional (2D) surface. If this surface is folded along a third dimension, it allows one to picture a wormhole "bridge". (This is merely a visualization displayed to convey an essentially unvisualisable structure existing in 4 or more dimensions. The parts of the wormhole could be higher-dimensional analogues for the parts of the curved 2D surface; for example, instead of mouths which are circular holes in a 2D plane, a real wormhole's mouths could be spheres in 3D space. A wormhole is, in theory, much like a tunnel with two ends each in separate points in spacetime similar analysis can be used to explain Hawking radiation that causes the slow "evaporation" of black holes (although this is generally visualized as the escape of one particle from avirtual particle-antiparticle pair(PAIR LOSE), the other particle having been captured by the black hole(GAINS).

\section{AGE OF VACUUM ENERGY IN A RADIATION FILLED DESITTER UNIVERSE}

So this is the de Sitter universe, and that it is also used as an approximation to inflationary models whose dynamics are similar. Is this idea of "no beginning" sort of the basis for "eternal inflation? The only issue here is that any amount of matter or radiation causes the universe to have a finite age. So it is not considered feasible for inflation to be past-eternal, because there will always be some matter or radiation, no matter how diffuse

Inflation and Vacuum Energy Density-its effects on the classification of Vacuum energy:

The "inflationary scenario", developed by Starobinsky and by Guth, offers a solution to the flatness-oldness problem and the horizon problem. The inflationary scenario invokes a vacuum energy density. We normally think of the vacuum as empty and massless, and we can determine that the density of the vacuum is less than $10^{-29} \mathrm{gm} / \mathrm{cc}$ now. But in quantum field theory, the vacuum is not empty, but rather filled with virtual particles 


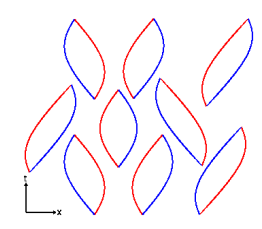

The space-time diagram above shows virtual particle-antiparticle pairs forming out of nothing and then annihilating back into nothing. For particles of mass $\mathrm{m}$, one expects about one virtual particle in each cubical volume with sides given by the Compton wavelength of the particle, $\mathrm{h} / \mathrm{mc}$, where $\mathrm{h}$ is Planck's constant. Thus the expected density of the vacuum is rho $=\mathrm{m}^{4} * \mathrm{c}^{3} / \mathrm{h}^{3}$ which is rather large. For the largest elementary particle mass usually considered, the Planck mass $M$ defined by $2 * \mathrm{pi}^{*} \mathrm{G}^{*} \mathrm{M}^{2}=\mathrm{h} *$, this density is $2 * 10^{91} \mathrm{gm} / \mathrm{cc}$. That's a 2 followed by 91 zeroes! Thus the vacuum energy density is at least 120 orders of magnitude smaller than the naive quantum estimate, so there must be a very effective suppression

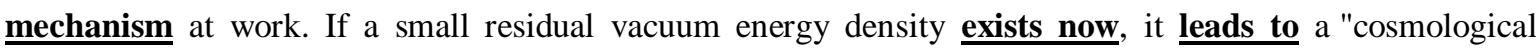
constant" which is one proposed mechanism to relieve the tight squeeze between the Omega $=1$ model age of the Universe, $t_{0}=(2 / 3) / H_{0}=9$ Gyr, and the apparent age of the oldest globular clusters, 12-14 Gyr. The vacuum energy density can do this because it produces a "repulsive gravity" that causes the expansion of the Universe to accelerate instead of decelerate, and this increases $t_{0}$ for a given $\mathrm{H}_{0}$.

The inflationary scenario proposes that the vacuum energy was very large during a brief period early in the history of the Universe. When the Universe is dominated by a vacuum energy density the scale factor grows exponentially, a $(\mathrm{t})=\exp \left(\mathrm{H}\left(\mathrm{t}-\mathrm{t}_{\mathrm{o}}\right)\right)$. The Hubble constant really is constant during this epoch so it doesn't need the "naught". If the inflationary epoch lasts long enough the exponential function gets very large. This makes a ( $t$ ) very large, and thus makes the radius of curvature of the Universe very large. The diagram below shows our horizon superimposed on a very large radius sphere on top, or a smaller sphere on the bottom. Since we can only see as far as our horizon, for the inflationary case on top the large radius sphere looks almost flat to us. This is how Hawking also explains the connected and disconnected Euclidean matrices.

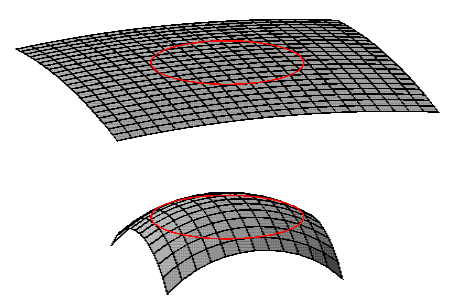

This solves the ' flatness-oldness problem' as long as the 'exponential growth during the inflationary epoch' continues for at least 100 doublings. Inflation also solves the horizon problem, because the future light cone of an event that happens before inflation is expanded to a huge region by the growth during inflation. 


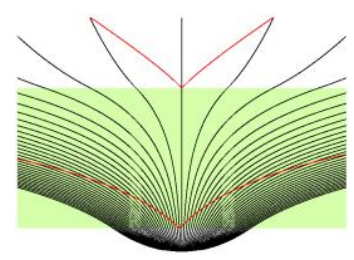

This space-time diagram shows the inflationary epoch tinted green, and the future light cones of two events in red. The early event has a future light cone that covers a huge area that can easily encompass our entire horizon. Thus we can explain why the temperature of the microwave background is so uniform across the sky.

Large-Scale Structure and Anisotropy and Vacuum Energy.

Of course the Universe is not really homogeneous, since it contains dense regions like galaxies and people. Dense regions produce heterogeneity. These dense regions should affect the temperature of the microwave background. Sachs and Wolfe (1967, ApJ, 147, 73) derived the effect of the gravitational potential perturbations on the CMB. The gravitational potential, phi $=-\mathrm{GM} / \mathrm{r}$, will be negative in dense lumps, and positive in less dense regions. Photons lose energy when they climb out of the gravitational potential wells of the lumps:

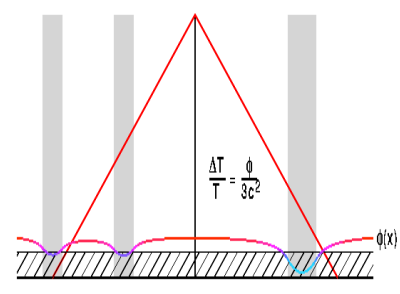

This conformal space-time diagram above shows lumps as gray vertical bars, the epoch before recombination as the hatched region, and the gravitational potential as the color-coded curve phi(x). Where our past light cone intersects the surface of recombination, we see a temperature perturbed by $\mathrm{dT} / \mathrm{T}=$ $\mathrm{phi} /\left(3^{*} \mathrm{c}^{2}\right)$. Sachs and Wolfe predicted temperature fluctuations $\mathrm{dT} / \mathrm{T}$ as large as 1 percent, but we know now that the Universe is far more homogeneous than Sachs and Wolfe thought. So observers worked for years to get enough sensitivity to see the temperature differences around the sky. The first anisotropy to be detected was the dipole anisotropy by Conklin in 1969: Part of the sky is hotter by (v/c)*to, while the blue part of the sky is colder by $(\mathrm{v} / \mathrm{c}) *$ to, where the inferred velocity is $\mathrm{v}=368 \mathrm{~km} / \mathrm{sec}$. This is how we measure the velocity of the Solar System relative to the observable Universe. It was another 23 years before the anisotropy predicted by Sachs and Wolfe was detected by Smoot et al. (1992, ApJL, 396, 1). The amplitude was 1 part in 100,000 instead of 1 part in 100, but was perfectly consistent with LambdaCDM [Wright et al. 1992, ApJL, 396, 13].

\section{Cosmic anisotropy and Void:}

Cosmic anisotropy (and detector noise) after the dipole pattern and the radiation from the Milky Way have been subtracted out. The anisotropy if converted into a gravitational potential using Sachs and Wolfe's result and that potential is then expressed as a height assuming a constant acceleration of gravity equal to the gravity on the Earth, we get a height of twice the distance from the Earth to the Sun. The "mountains and valleys" of the Universe are really quite large. Inflation predicts a certain statistical pattern in the anisotropy. The quantum fluctuations normally affect very small regions of space, but the huge exponential expansion during the inflationary epoch makes these tiny regions observable. The pattern formed by adding all of the effects from events of all ages is known as "equal power on all scales", and it agrees with the COBE data. Having found that the observed pattern of anisotropy is consistent with inflation, we can also ask whether the amplitude implies gravitational forces large enough to produce the observed clustering of galaxies. The conformal space-time diagram above shows the phi(x) at recombination determined by COBE's dT data, and the worldliness of galaxies which are perturbed by the gravitational forces produced by the gradient of the potential. Matter flows "downhill" away from peaks of the potential 
producing voids in the current distribution of galaxies, while valleys in the potential (blue spots) are where the clusters of galaxies form.

\section{ENERGY DENSITY IN THE UNIVERSE IS VACUUM DENSITY}

Gravitational forces are large enough to produce the observed clustering, but only if these forces are not opposed by other forces. If the all the matter in the Universe is made out of the ordinary chemical elements, then there was a very effective opposing force before recombination, because the free electrons which are now bound into atoms were very effective at scattering the photons of the cosmic background. We can therefore conclude that most of the matter in the Universe is "dark matter" that does not emit, absorb or scatter light. Furthermore, observations of distant supernovae have shown that most of the energy density of the Universe is a vacuum energy density (a "dark energy") like Einstein's cosmological constant that causes an accelerating expansion of the Universe. These strange conclusions have been greatly strengthened by temperature anisotropy data at smaller angular scales which was provided by the Wilkinson Microwave Anisotropy Probe (WMAP) in 2003.

\section{Pressure less matter $\left(\rho^{\rho} \propto R^{-3}\right)$, radiation $\left({ }^{\rho} \propto R^{-4}\right)$ and vacuum energy}

The equation of motion for the scale factor can be obtained in a quasi-Newtonian fashion. Consider a sphere about some arbitrary point, and let the radius be $R(t) r$, where $r$ is arbitrary. The motion of a point at the edge of the sphere will, in Newtonian gravity, be influenced only by the interior mass. We can therefore write down immediately a differential equationFriedmann's equation) that expresses conservation of energy: $\dot{r} r$ ) $2 / 2-G M /(R r)=$ constant. In fact, to get this far, we do require general relativity: the gravitation from mass shells at large distances is not Newtonian, and so we cannot employ the usual argument about their effect being zero. In fact, the result that the gravitational field inside a uniform shell is zero does hold in general relativity, and is known as Birkhoff's theorem . General relativity becomes even more vital in giving us the constant of integration in Friedman's equation The Friedman equation shows that a universe that is spatially closed (with $k=+1$ ) has negative total "energy": the expansion will eventually be halted by gravity, and the universe will recollapse. Conversely, an unbound model is spatially open $(k=-1)$ and will expand forever. This is marvelously simple: the dynamics of the entire universe are the same as those of a cannonball fired vertically against the Earth's gravity. Just as the Earth's gravity defines an escape velocity for projectiles, so a universe that expands sufficiently fast will continue to expand forever. Conversely, for a given rate of expansion there is a critical density that will bring the expansion asymptotically to a halt

$$
\rho_{c}=\frac{3 H^{2}}{8 \pi G}
$$

This connection between the rate of expansion of the universe and its global geometry is deep result. An alternative line of attack is to rewrite the Friedmann equation in terms of the Hubble parameter:

$$
H^{2}-\frac{8 \pi G}{3} \rho=\frac{\text { constant }}{R^{2}}
$$

The "flat" universe with $k=0$ arises for a particular critical density. We are therefore led to define a density parameter as the ratio of density to critical density:

$$
\Omega \equiv \frac{\rho}{\rho_{c}}=\frac{8 \pi G \rho}{3 H^{2}} .
$$

Since ${ }^{\rho}$ and $H$ change with time, this defines an epoch-dependent density parameter. The current value of the parameter should strictly be denoted by $\omega_{0}$. Pressureless matter $\left({ }^{\rho} \propto R^{-3}\right)$, radiation $\left({ }^{\rho} \propto R^{-4}\right)$ and vacuum energy ( $\rho_{\text {constant Note that the transformations and transactions take place continuously }}$ 
depite the constant state of the Vacuum energy).is what universe could be divided in to. The first two relations just say that the number density of particles is diluted by the expansion, with photons also having their energy reduced by the redshift; the third relation applies for Einstein's cosmological constant. The Hubble constant thus sets the curvature length, which becomes infinitely large as $\omega$ approaches unity from either direction. Only in the limit of zero density does this length become equal to the other common measure of the size of the universe - the Hubble length, $c / H_{0} \cdot t^{2 / 3}$.

One of the great conclusions of relativistic cosmology: the universe is of finite age, and had its origin in a mathematical singularity at which the scale factor went to zero, leading to a divergent spacetime curvature. Since zero scale factor also implies infinite density (and temperature), the inferred(manifested) picture of the early universe is one of unimaginable violence tempestuous and turbulent internal differentiation and comparative variability and structural morphology and within normative aspect of expectations usually drawn by cosmologists. The term big bang was coined by Fred Hoyle to describe this beginning, although it was intended somewhat critically. The problem with the singularity is that it marks the breakdown of the laws of physics; we cannot extrapolate the solution for $R(t)$ to $t<0$, and so the origin of the expansion becomes an unexplained boundary condition

MASS DENSITY AND VACUUM ENERGY -THE REPULSIVE COUNTERBALANCING FORCE: Since ${ }^{P}=-p$ for vacuum energy, and this is the only source of pressure if we ignore radiation, this tells us that ${ }^{P}=3^{P}$ and hence that the mass density is twice the vacuum density. The total density is hence positive and $k=1$; we have a closed model. Notice that what this says is that a positive vacuum energy acts in a repulsive way, balancing the attraction of normal matter. This is related to the idea of ${ }^{p}+3 p$ as the effective source density for gravity. This insight alone should make one appreciate that the static model cannot be stable: if we perturb the scale factor by a small positive amount, the vacuum repulsion is unchanged whereas the "normal" gravitational attraction is reduced, so that the model will tend to expand further (or contract, if the initial perturbation was negative). Thinking along these lines, a tidy history of science would have required Einstein to predict the expanding universe in advance of its observation.

\section{VACUUM ENERGY AND DESITTER SPACE:}

DE SITTER SPACE Before going on to the general case, it is worth looking at the endpoint of an outwards perturbation of Einstein's static model, first studied by de Sitter and named after him. This universe is completely dominated by vacuum energy, and is clearly the limit of the unstable expansion, since the density of matter redshift to zero while the vacuum energy remains constant. It was thought that the cosmological constant is what caused the expansion. He tells us that the Hubble constant really is constant, and so the model necessarily has exponential expansion, $R \boldsymbol{\alpha} \exp (H t)$, exactly as for de Sitter space. Furthermore, it is necessary that $k=0$, as may be seen by considering the transverse part of the RobertsonWalker metric: $d \sigma^{2}=\left[R(t) S_{k}(r) d \psi\right]^{2}$. This has the convention that $r$ is a dimensionless commoving coordinate; if we divide by $R_{0}$ and change to physical radius $r^{\prime}$, the metric becomes $d \sigma^{2}=\left[a(t) R_{0} S_{k}\left(r^{\prime}\right)\right.$ $\left.\left.R_{0}\right) d \Psi\right]^{2}$. The current scale factor $R_{0}$ now plays the role of a curvature length, determining the distance over which the model is spatially Euclidean. However, any such curvature radius must be constant in the steadystate model, so the only possibility is that it is infinite and that $k=0$. We thus see that de Sitter space is a steady-state universe: it contains a constant vacuum energy density, and has an infinite age, lacking any big-bang singularity. In this sense, some aspects of the steady-state model have been resurrected in inflationary cosmology. However, de Sitter space is a rather uninteresting model because it contains no matter. Introducing matter into a steady-state universe violates energy conservation, since matter does not have the $p=-\rho_{c} c^{2}$ equation of state that allows the density to remain constant. This is the most radical aspect of steady-state models: they require continuous creation of matter. (Carroll, Press \& Turner 1992

If the density is below the critical density, the result would be that the universe will expand forever, at an ever decreasing rate; the universe will be open. In this case its global geometry will have negative curvature, like the surface of a trumpet. The angles of a large triangle add up to less that $180^{\circ}$.If the density is exactly equal to the critical density the universe will have a flat geometry, and universe will expand forever at an ever decreasing rate. The angles of all triangles add up to $180^{\circ}$. 


\section{STABILITY ANALYSIS:}

From the above stability analysis we infer the following:

1. The adjustment process is stable in the sense that the system of oxygen consumption converges to equilibrium.

2.The approach to equilibrium is a steady one, and there exists progressively diminishing oscillations around the equilibrium point

3.Conditions 4 and 2 are independent of the size and direction of initial disturbance

4.The actual shape of the time path of VE is determined by efficiency parameter, the strength of the response of the portfolio in question, and the initial disturbance5.Result 3 warns us that we need to make an exhaustive study of the behavior of any case in which generalization derived from the model do not hold

6.Growth studies as the one in the extant context are related to the systemic growth paths with full employment of resources that are available in question, in the present case terrestrial organisms -oxygen consumption-dead organic matter and decomposer organisms

7. Some authors were interested in such questions, whether growing system could produce full employment of all factors, whether or not there was a full employment natural rate growth path and perpetual oscillations around it. It is to be noted some systems pose extremely difficult stability problems. As an instance, one can quote example of pockets of PRIGOGINE'S DISSIPATIVE STRCTURES like open cells and drizzle in complex networks in marine stratocumulus. Other examples are clustering and synchronization of lightning flashes adjunct to thunderstorms, coupled studies of microphysics and aqueous chemistry.

\section{QUANTUM FIELD(QF) PORTFOLIO::}

Assumptions:

(1)Category 1 is representative of QF vis-à-vis VE category 1

(2)Category 2 constitutes QF in correspondence with the similar classification of QF(3) Category 3 of QF with respect to category 3 of $\mathrm{VE}$

(a)The speed of growth of QF category 1 is a linear function of that sector in category 2 at the time of reckoning. As before the accentuation coefficient that characterizes the speed of growth in category 1 is the proportionality factor between balance in category 1 and category 2 .

(b)The dissipation coefficient in the growth model is attributable to two factors;(1) With the progress of time terrestrial organism sector gets aged and become eligible for transfer to the next category. Notwithstanding Category 3 does not have such a provision for further transference provisionalities

CInflow into category 2 is only from category 1 in the form of transfer of balance of QF from the category 1.This is evident from the age wise classification scheme. As a result, the speed of growth of category 2 is dependent upon the amount of inflow, which is a function of the quantum of balance of terrestrial organism sector under the category 1 .The balance of QF sector in category 3 is because of transfer of balance from category 2. It is dependent on the amount of QF (See above discussion of energy density of matter both in classical and quantum mechanics) sector under category 2. 
NOTATION :

$T_{24}$ : Balance standing in the category 1 of $\mathrm{QF}$

$T_{25}$ : Balance standing in the category 2 of $\mathrm{QF}$

$T_{26}$ : Balance standing in the category 3 of $\mathrm{QF}$

$\left(b_{24}\right)^{(4)},\left(b_{25}\right)^{(4)},\left(b_{26}\right)^{(4)}:$ Accentuation coefficients

$$
\left(b_{24}^{\prime}\right)^{(4)},\left(b_{25}^{\prime}\right)^{(4)},\left(b_{26}^{\prime}\right)^{(4)} \text { : Dissipation coefficients }
$$

\section{FORMULATION OF THE SYSTEM :}

Under the above assumptions, we derive the following :

a) The growth speed in category 1 is the sum of two parts:

1. A term $+\left(b_{24}\right)^{(4)} T_{25}$ proportional to the amount of balance of the category 2

2. A term $-\left(b_{24}^{\prime}\right)^{(4)} T_{24}$ representing the quantum of balance dissipated from category 1 .

b) The growth speed in category 2 is the sum of two parts:

1. A term $+\left(b_{25}\right)^{(4)} T_{24}$ constitutive of the amount of inflow from the category

2. A term $-\left(b_{25}^{\prime}\right)^{(4)} T_{25}$ the dissipation factor

The growth speed under category 3 is attributable to inflow from category 2 and any other dissipations in QF

\section{GOVERNING EQUATIONS:}

Following are the differential equations that govern the growth in the terrestrial organisms portfolio

$$
\begin{aligned}
& \frac{d T_{24}}{d t}=\left(b_{24}\right)^{(4)} T_{25}-\left(b_{24}^{\prime}\right)^{(4)} T_{24} \\
& \frac{d T_{25}}{d t}=\left(b_{25}\right)^{(4)} T_{24}-\left(b_{25}^{\prime}\right)^{(4)} T_{25} \\
& \frac{d T_{26}}{d t}=\left(b_{26}\right)^{(4)} T_{25}-\left(b_{26}^{\prime}\right)^{(4)} T_{26}
\end{aligned}
$$

$\left(b_{i}\right)^{(4)}>0 \quad, \quad i=24,25,26$

$\left(b_{i}^{\prime}\right)^{(4)}>0, \quad i=24,25,26$

$$
\begin{aligned}
& \left(b_{25}\right)^{(4)}<\left(b_{24}^{\prime}\right)^{(4)} \\
& \left(b_{26}\right)^{(4)}<\left(b_{25}^{\prime}\right)^{(4)}
\end{aligned}
$$

Following the same procedure outlined in the previous section , the general solution of the governing equations is $\alpha_{i}^{\prime} T_{i}+\beta_{i}^{\prime} T_{i}+\gamma_{i}^{\prime} T_{i}=C_{i}^{\prime} e_{i}{ }^{\prime}{ }^{t} t, i=24,25,26$ where $C_{24}^{\prime}, C_{25}^{\prime}, C_{26}^{\prime}$ are arbitrary constant coefficients and $\alpha_{24}^{\prime}, \alpha_{25}^{\prime}, \alpha_{26}^{\prime}, \gamma_{24}^{\prime}, \gamma_{25}^{\prime}, \gamma_{26}^{\prime}$ corresponding multipliers to the characteristic roots of the system

\section{VACUUM ENERGY(VE) AND QUANTUM FIELD(QF) SYSTEM-INTERPRETATION OF DUAL SYSTEM}


We will denote

1) By $T_{i}(t), i=24,25,26$, the three parts of the QF analogously to the $G_{i}$ of the VE

2) $\left(a_{i}^{\prime \prime}\right)^{(4)}\left(T_{25}, t\right)\left(T_{25} \geq 0, t \geq 0\right)$, the contribution of the $\mathrm{QF}$ to the dissipation coefficient of the VE

3) By $\left(-b_{i}^{\prime \prime}\right)^{(4)}\left(G_{24}, G_{25}, G_{26}, t\right)=-\left(b_{i}^{\prime \prime}\right)^{(4)}\left(\left(G_{27}\right), t\right)$, the contribution of the VE to the dissipation coefficient of the QF

GOVERNING EQUATIONS:

The differential system of this model is now

$$
\begin{aligned}
& \frac{d G_{24}}{d t}=\left(a_{24}\right)^{(4)} G_{25}-\left[\left(a_{24}^{\prime}\right)^{(4)}+\left(a_{24}^{\prime \prime}\right)^{(4)}\left(T_{25}, t\right)\right] G_{24} \\
& \frac{d G_{25}}{d t}=\left(a_{25}\right)^{(4)} G_{24}-\left[\left(a_{25}^{\prime}\right)^{(4)}+\left(a_{25}^{\prime \prime}\right)^{(4)}\left(T_{25}, t\right)\right] G_{25} \\
& \frac{d G_{26}}{d t}=\left(a_{26}\right)^{(4)} G_{25}-\left[\left(a_{26}^{\prime}\right)^{(4)}+\left(a_{26}^{\prime \prime}\right)^{(4)}\left(T_{25}, t\right)\right] G_{26} \\
& \frac{d T_{24}}{d t}=\left(b_{24}\right)^{(4)} T_{25}-\left[\left(b_{24}^{\prime}\right)^{(4)}-\left(b_{24}^{\prime \prime}\right)^{(4)}\left(\left(G_{27}\right), t\right)\right] T_{24} \\
& \frac{d T_{25}}{d t}=\left(b_{25}\right)^{(4)} T_{24}-\left[\left(b_{25}^{\prime}\right)^{(4)}-\left(b_{25}^{\prime \prime}\right)^{(4)}\left(\left(G_{27}\right), t\right)\right] T_{25} \\
& \frac{d T_{26}}{d t}=\left(b_{26}\right)^{(4)} T_{25}-\left[\left(b_{26}^{\prime}\right)^{(4)}-\left(b_{26}^{\prime \prime}\right)^{(4)}\left(\left(G_{27}\right), t\right)\right] T_{26} \\
&+\left(a_{24}^{\prime \prime}\right)^{(4)}\left(T_{25}, t\right)=\text { First augmentation factor DUE TO QF }
\end{aligned}
$$

$-\left(b_{24}^{\prime \prime}\right)^{(4)}(G, t)=$ First detritions factor contributed by VE to the dissipation of QF

Where we suppose

$$
\left(a_{i}\right)^{(4)},\left(a_{i}^{\prime}\right)^{(4)},\left(a_{i}^{\prime \prime}\right)^{(4)},\left(b_{i}\right)^{(4)},\left(b_{i}^{\prime}\right)^{(4)},\left(b_{i}^{\prime \prime}\right)^{(4)}>0, \quad i, j=24,25,26
$$

(A) The functions $\left(a_{i}^{\prime \prime}\right)^{(4)},\left(b_{i}^{\prime \prime}\right)^{(4)}$ are positive continuous increasing and bounded.

$$
\begin{aligned}
& \text { Definition of }\left(p_{i}\right)^{(4)},\left(r_{i}\right)^{(4)} \text { : } \\
& \left(a_{i}^{\prime \prime}\right)^{(4)}\left(T_{25}, t\right) \leq\left(p_{i}\right)^{(4)} \leq\left(\hat{A}_{24}\right)^{(4)} \\
& \left(b_{i}^{\prime \prime}\right)^{(4)}\left(\left(G_{27}\right), t\right) \leq\left(r_{i}\right)^{(4)} \leq\left(b_{i}^{\prime}\right)^{(4)} \leq\left(\hat{B}_{24}\right)^{(4)} \\
& \text { (B) } \quad \lim _{T_{2} \rightarrow \infty}\left(a_{i}^{\prime \prime}\right)^{(4)}\left(T_{25}, t\right)=\left(p_{i}\right)^{(4)} \\
& \lim _{\mathrm{G} \rightarrow \infty}\left(b_{i}^{\prime \prime}\right)^{(4)}\left(G_{27}, t\right)=\left(r_{i}\right)^{(4)}
\end{aligned}
$$

Where $\left(\hat{A}_{24}\right)^{(4)},\left(\hat{B}_{24}\right)^{(4)},\left(p_{i}\right)^{(4)},\left(r_{i}\right)^{(4)}$ are positive constants and $i=24,25,26$ 
They satisfy Lipschitz condition:

$$
\begin{gathered}
\left|\left(a_{i}^{\prime \prime}\right)^{(4)}\left(T_{25}^{\prime}, t\right)-\left(a_{i}^{\prime \prime}\right)^{(4)}\left(T_{25}, t\right)\right| \leq\left(\hat{k}_{24}\right)^{(4)}\left|T_{25}-T_{25}^{\prime}\right| e^{-\left(\widehat{M}_{24}\right)^{(4)} t} \\
\left|\left(b_{i}^{\prime \prime}\right)^{(4)}\left(\left(G_{27}\right)^{\prime}, t\right)-\left(b_{i}^{\prime \prime}\right)^{(4)}\left(\left(G_{27}\right), T\right)\right|<\left(\hat{k}_{24}\right)^{(4)}||\left(G_{27}\right)-\left(G_{27}\right)^{\prime}|| e^{-\left(M_{24}\right)^{(4)} t}
\end{gathered}
$$

With the Lipschitz condition, we place a restriction on the behavior of functions $\left(a_{i}^{\prime \prime}\right)^{(4)}\left(T_{25}^{\prime}, t\right)$ $\operatorname{and}\left(a_{i}^{\prime \prime}\right)^{(4)}\left(T_{25}, t\right) \quad\left(T_{25}^{\prime}, t\right)$ And $\left(T_{25}, t\right)$ are points belonging to the interval $\left[\left(\hat{k}_{24}\right)^{(4)},\left(\widehat{M}_{24}\right)^{(4)}\right]$. It is to be noted that $\left(a_{i}^{\prime \prime}\right)^{(4)}\left(T_{25}, t\right)$ is uniformly continuous. In the eventuality of the fact, that if $\left(\widehat{M}_{24}\right)^{(4)}=4$ then the function $\left(a_{i}^{\prime \prime}\right)^{(4)}\left(T_{25}, t\right)$, the first augmentation coefficient, would be absolutely continuous.

Definition of $\left(\widehat{M}_{24}\right)^{(4)},\left(\hat{k}_{24}\right)^{(4)}$ :

$\left(\widehat{M}_{24}\right)^{(4)},\left(\hat{k}_{24}\right)^{(4)}$, are positive constants

$$
\frac{\left(a_{i}\right)^{(4)}}{\left(\widehat{M}_{24}\right)^{(4)}}, \frac{\left(b_{i}\right)^{(4)}}{\left(\widehat{M}_{24}\right)^{(4)}}<1
$$

Definition of $\left(\hat{P}_{24}\right)^{(4)},\left(\hat{Q}_{24}\right)^{(4)}$ :

(C) There exists two constants $\left(\hat{P}_{24}\right)^{(4)}$ and $\left(\hat{Q}_{24}\right)^{(4)}$ which together with $\left(\widehat{M}_{24}\right)^{(4)},\left(\hat{k}_{24}\right)^{(4)},\left(\hat{A}_{24}\right)^{(4)}$ and $\left(\hat{B}_{24}\right)^{(4)} \quad$ and $\quad$ the constants $\left(a_{i}\right)^{(4)},\left(a_{i}^{\prime}\right)^{(4)},\left(b_{i}\right)^{(4)},\left(b_{i}^{\prime}\right)^{(4)},\left(p_{i}\right)^{(4)},\left(r_{i}\right)^{(4)}, i=24,25,26$,

satisfy the inequalities

$$
\begin{aligned}
\frac{1}{\left(\widehat{M}_{24}\right)^{(4)}}\left[\left(a_{i}\right)^{(4)}+\left(a_{i}^{\prime}\right)^{(4)}+\left(\hat{A}_{24}\right)^{(4)}+\left(\hat{P}_{24}\right)^{(4)}\left(\hat{k}_{24}\right)^{(4)}\right]<1 \\
\frac{1}{\left(\widehat{M}_{24}\right)^{(4)}}\left[\left(b_{i}\right)^{(4)}+\left(b_{i}^{\prime}\right)^{(4)}+\left(\hat{B}_{24}\right)^{(4)}+\left(\hat{Q}_{24}\right)^{(4)}\left(\hat{k}_{24}\right)^{(4)}\right]<1
\end{aligned}
$$

Theorem 4: if the conditions (A)-(E) above are fulfilled, there exists a solution satisfying the conditions

Definition of $G_{i}(0), T_{i}(0)$ :

$$
\begin{array}{ll}
G_{i}(t) \leq\left(\hat{P}_{24}\right)^{(4)} e^{\left(M_{24}\right)^{(4)} t}, & G_{i}(0)=G_{i}^{0}>0 \\
T_{i}(t) \leq\left(\hat{Q}_{24}\right)^{(4)} e^{\left(M_{24}\right)^{(4)} t}, & T_{i}(0)=T_{i}^{0}>0
\end{array}
$$

\section{Proof:}

Consider operator $\mathcal{A}^{(4)}$ defined on the space of sextuples of continuous functions $G_{i}, T_{i}: \mathbb{R}_{+} \rightarrow \mathbb{R}_{+}$ which satisfy

$$
G_{i}(0)=G_{i}^{0}, T_{i}(0)=T_{i}^{0}, G_{i}^{0} \leq\left(\hat{P}_{24}\right)^{(4)}, T_{i}^{0} \leq\left(\hat{Q}_{24}\right)^{(4)},
$$




$$
\begin{aligned}
& 0 \leq G_{i}(t)-G_{i}^{0} \leq\left(\hat{P}_{24}\right)^{(4)} e^{\left(M_{24}\right)^{(4)} t} \\
& 0 \leq T_{i}(t)-T_{i}^{0} \leq\left(\hat{Q}_{24}\right)^{(4)} e^{\left(M_{24}\right)^{(4)} t}
\end{aligned}
$$

By

$$
\begin{gathered}
\left.\bar{G}_{24}(t)=G_{24}^{0}+\int_{0}^{t}\left[\left(a_{24}\right)^{(4)} G_{25}\left(s_{(24)}\right)-\left(\left(a_{24}^{\prime}\right)^{(4)}+a_{24}^{\prime \prime}\right)^{(4)}\left(T_{25}\left(s_{(24)}\right), s_{(24)}\right)\right) G_{24}\left(s_{(24)}\right)\right] d s_{(24)} \\
\bar{G}_{25}(t)=G_{25}^{0}+\int_{0}^{t}\left[\left(a_{25}\right)^{(4)} G_{24}\left(s_{(24)}\right)-\left(\left(a_{25}^{\prime}\right)^{(4)}+\left(a_{25}^{\prime \prime}\right)^{(4)}\left(T_{25}\left(s_{(24)}\right), s_{(24)}\right)\right) G_{25}\left(s_{(24)}\right)\right] d s_{(24)} \\
\bar{G}_{26}(t)=G_{26}^{0}+\int_{0}^{t}\left[\left(a_{26}\right)^{(4)} G_{25}\left(s_{(24)}\right)-\left(\left(a_{26}^{\prime}\right)^{(4)}+\left(a_{26}^{\prime \prime}\right)^{(4)}\left(T_{25}\left(s_{(24)}\right), s_{(24)}\right)\right) G_{26}\left(s_{(24)}\right)\right] d s_{(24)} \\
\bar{T}_{24}(t)=T_{24}^{0}+\int_{0}^{t}\left[\left(b_{24}\right)^{(4)} T_{25}\left(s_{(24)}\right)-\left(\left(b_{24}^{\prime}\right)^{(4)}-\left(b_{24}^{\prime \prime}\right)^{(4)}\left(G\left(s_{(24)}\right), s_{(24)}\right)\right) T_{24}\left(s_{(24)}\right)\right] d s_{(24)} \\
\bar{T}_{25}(t)=T_{25}^{0}+\int_{0}^{t}\left[\left(b_{25}\right)^{(4)} T_{24}\left(s_{(24)}\right)-\left(\left(b_{25}^{\prime}\right)^{(4)}-\left(b_{25}^{\prime \prime}\right)^{(4)}\left(G\left(s_{(24)}\right), s_{(24)}\right)\right) T_{25}\left(s_{(24)}\right)\right] d s_{(24)} \\
\bar{T}_{26}(\mathrm{t})=\mathrm{T}_{26}^{0}+\int_{0}^{t}\left[\left(b_{26}\right)^{(4)} T_{25}\left(s_{(24)}\right)-\left(\left(b_{26}^{\prime}\right)^{(4)}-\left(b_{26}^{\prime \prime}\right)^{(4)}\left(G\left(s_{(24)}\right), s_{(24)}\right)\right) T_{26}\left(s_{(24)}\right)\right] d s_{(24)}
\end{gathered}
$$

Where $s_{(24)}$ is the integrand that is integrated over an interval $(0, t)$

(a) The operator $\mathcal{A}^{(4)}$ maps the space of functions satisfying 34,35,36 into itself .Indeed it is obvious that

$$
\begin{gathered}
G_{24}(t) \leq G_{24}^{0}+\int_{0}^{t}\left[\left(a_{24}\right)^{(4)}\left(G_{25}^{0}+\left(\hat{P}_{24}\right)^{(4)} e^{\left.\left(\widehat{M}_{24}\right)^{(4)} s_{(24)}\right)}\right)\right] d s_{(24)}= \\
\left(1+\left(a_{24}\right)^{(4)} t\right) G_{25}^{0}+\frac{\left(a_{24}\right)^{(4)}\left(\hat{P}_{24}\right)^{(4)}}{\left(\widehat{M}_{24}\right)^{(4)}}\left(e^{\left(\widehat{M}_{24}\right)^{(4)} t}-1\right)
\end{gathered}
$$

From which it follows that

$$
\left(G_{24}(t)-G_{24}^{0}\right) e^{-\left(M_{24}\right)^{(4)} t} \leq \frac{\left(a_{24}\right)^{(4)}}{\left(\widehat{M}_{24}\right)^{(4)}}\left[\left(\left(\hat{P}_{24}\right)^{(4)}+G_{25}^{0}\right) e^{\left(-\frac{\left(\hat{P}_{24}\right)^{(4)}+G_{25}^{0}}{G_{25}^{0}}\right)}+\left(\hat{P}_{24}\right)^{(4)}\right]
$$

$\left(G_{i}^{0}\right)$ is as defined in the statement of theorem 1

Analogous inequalities hold also for $G_{25}, G_{26}, T_{24}, T_{25}, T_{26}$

It is now sufficient to take $\frac{\left(a_{i}\right)^{(4)}}{\left(M_{24}\right)^{(4)}}, \frac{\left(b_{i}\right)^{(4)}}{\left(M_{24}\right)^{(4)}}<1$ and to choose

$\left(\widehat{\mathrm{P}}_{24}\right)^{(4)}$ and $\left(\widehat{\mathrm{Q}}_{24}\right)^{(4)}$ large to have 


$$
\begin{aligned}
& \frac{\left(a_{i}\right)^{(4)}}{\left(\widehat{M}_{24}\right)^{(4)}}\left[\left(\widehat{P}_{24}\right)^{(4)}+\left(\left(\hat{P}_{24}\right)^{(4)}+G_{j}^{0}\right) e^{-\left(\frac{\left(\hat{P}_{24}\right)^{(4)}+G_{j}^{0}}{G_{j}^{0}}\right)}\right] \leq\left(\hat{P}_{24}\right)^{(4)} \\
& \frac{\left(b_{i}\right)^{(4)}}{\left(\widehat{M}_{24}\right)^{(4)}}\left[\left(\left(\hat{Q}_{24}\right)^{(4)}+T_{j}^{0}\right) e^{-\left(\frac{\left(\hat{Q}_{24}\right)^{(4)}+T_{j}^{0}}{T_{j}^{0}}\right)}+\left(\hat{Q}_{24}\right)^{(4)}\right] \leq\left(\hat{Q}_{24}\right)^{(4)}
\end{aligned}
$$

In order that the operator $\mathcal{A}^{(4)}$ transforms the space of sextuples of functions $G_{i}, T_{i}$ satisfying 34,35,36 into itself

The operator $\mathcal{A}^{(4)}$ is a contraction with respect to the metric

$$
\begin{gathered}
d\left(\left(\left(G_{27}\right)^{(1)},\left(T_{27}\right)^{(1)}\right),\left(\left(G_{27}\right)^{(2)},\left(T_{27}\right)^{(2)}\right)\right)= \\
\sup _{i}\left\{\max _{t \in \mathbb{R}_{+}}\left|G_{i}^{(1)}(t)-G_{i}^{(2)}(t)\right| e^{-\left(\widehat{M}_{24}\right)^{(4)} t}, \max _{t \in \mathbb{R}_{+}}\left|T_{i}^{(1)}(t)-T_{i}^{(2)}(t)\right| e^{-\left(\widehat{M}_{24}\right)^{(4)} t}\right\}
\end{gathered}
$$

Indeed if we denote

Definition of $\widetilde{\left(G_{27}\right)}, \widetilde{\left(T_{27}\right)}: \quad\left(\widetilde{\left(G_{27}\right)}, \widetilde{\left(T_{27}\right)}\right)=\mathcal{A}^{(4)}\left(\left(G_{27}\right),\left(T_{27}\right)\right)$

It results

$$
\begin{aligned}
& \left|\tilde{G}_{24}^{(1)}-\tilde{G}_{i}^{(2)}\right| \leq \int_{0}^{t}\left(a_{24}\right)^{(4)}\left|G_{25}^{(1)}-G_{25}^{(2)}\right| e^{-\left(\widehat{M}_{24}\right)^{(4)} s(24)} e^{\left(\widehat{M}_{24}\right)^{(4)} s_{(24)}} d s_{(24)}+ \\
& \int_{0}^{t}\left\{\left(a_{24}^{\prime}\right)^{(4)}\left|G_{24}^{(1)}-G_{24}^{(2)}\right| e^{-\left(\bar{M}_{24}\right)^{(4)} s(24)} e^{-\left(\bar{M}_{24}\right)^{(4)} s(24)}+\right. \\
& \left(a_{24}^{\prime \prime}\right)^{(4)}\left(T_{25}^{(1)}, s_{(24)}\right)\left|G_{24}^{(1)}-G_{24}^{(2)}\right| e^{-\left(\bar{M}_{24}\right)^{(4)} s_{(24)}} e^{\left(\widehat{M}_{24}\right)^{(4)} s_{(24)}}+ \\
& \left.G_{24}^{(2)}\left|\left(a_{24}^{\prime \prime}\right)^{(4)}\left(T_{25}^{(4)}, s_{(24)}\right)-\left(a_{24}^{\prime \prime}\right)^{(4)}\left(T_{25}^{(2)}, s_{(24)}\right)\right| e^{-\left(\mathbb{M}_{24}\right)^{(4)} s_{(24)}} e^{\left(M_{24}\right)^{(4)} s_{(24)}}\right\} d s_{(24)}
\end{aligned}
$$

Where $s_{(24)}$ represents integrand that is integrated over the interval $[0, \mathrm{t}]$

From the hypotheses on $25,26,27,28$ and 29 it follows

$$
\begin{aligned}
&\left|\left(G_{27}\right)^{(1)}-\left(G_{27}\right)^{(2)}\right| e^{-\left(\widehat{M}_{24}\right)^{(4)} t} \\
& \quad \leq \frac{1}{\left(\widehat{M}_{24}\right)^{(4)}}\left(\left(a_{24}\right)^{(4)}+\left(a_{24}^{\prime}\right)^{(4)}+\left(\widehat{A}_{24}\right)^{(4)}\right. \\
&\left.+\left(\widehat{P}_{24}\right)^{(4)}\left(\widehat{k}_{24}\right)^{(4)}\right) d\left(\left(\left(G_{27}\right)^{(1)},\left(T_{27}\right)^{(1)} ;\left(G_{27}\right)^{(2)},\left(T_{27}\right)^{(2)}\right)\right)
\end{aligned}
$$

And analogous inequalities for $G_{i}$ and $T_{i}$. Taking into account the hypothesis $(34,35,36)$ the result follows

Remark 4: The fact that we supposed $\left(a_{24}^{\prime \prime}\right)^{(4)}$ and $\left(b_{24}^{\prime \prime}\right)^{(4)}$ depending also on $\mathrm{t}$ can be considered as not conformal with the reality, however we have put this hypothesis, in order that we can postulate condition necessary to prove the uniqueness of the solution bounded by $\left(\widehat{P}_{24}\right)^{(4)} e^{\left(\widehat{M}_{24}\right)^{(4)} t}$ and $\left(\widehat{Q}_{24}\right)^{(4)} e^{\left(\bar{M}_{24}\right)^{(4)} t}$ respectively of $\mathbb{R}_{+}$. 
If instead of proving the existence of the solution on $\mathbb{R}_{+}$, we have to prove it only on a compact then it suffices to consider that $\left(a_{i}^{\prime \prime}\right)^{(4)}$ and $\left(b_{i}^{\prime \prime}\right)^{(4)}, i=24,25,26$ depend only on $\mathrm{T}_{25}$ and respectively on $\left(G_{27}\right)($ and not on $t$ ) and hypothesis can replaced by a usual Lipschitz condition.

Remark 2: There does not exist any $t$ where $G_{i}(t)=0$ and $T_{i}(t)=0$

From 19 to 24 it results

$$
\begin{aligned}
& G_{i}(t) \geq G_{i}^{0} e^{\left[-\int_{0}^{t}\left\{\left(a_{i}^{\prime}\right)^{(4)}-\left(a_{i}^{\prime \prime}\right)^{(4)}\left(T_{25}\left(s_{(24)}\right), s(24)\right)\right\} d s_{(24)}\right]} \geq 0 \\
& T_{i}(t) \geq T_{i}^{0} e^{\left(-\left(b_{i}^{\prime}\right)^{(4)} t\right)}>0 \text { for } \mathrm{t}>0
\end{aligned}
$$

Definition of $\left(\left(\widehat{M}_{24}\right)^{(4)}\right)_{1^{\prime}}\left(\left(\widehat{M}_{24}\right)^{(4)}\right)_{2}$ and $\left(\left(\widehat{M}_{24}\right)^{(4)}\right)_{3}$ :

Remark 3: if $G_{24}$ is bounded, the same property have also $G_{25}$ and $G_{26}$. indeed if $G_{24}<\left(\widehat{M}_{24}\right)^{(4)}$ it follows $\frac{d G_{25}}{d t} \leq\left(\left(\widehat{M}_{24}\right)^{(4)}\right)_{1}-\left(a_{25}^{\prime}\right)^{(4)} G_{25}$ and by integrating

$$
G_{25} \leq\left(\left(\widehat{M}_{24}\right)^{(4)}\right)_{2}=G_{25}^{0}+2\left(a_{25}\right)^{(4)}\left(\left(\widehat{M}_{24}\right)^{(4)}\right)_{1} /\left(a_{25}^{\prime}\right)^{(4)}
$$

In the same way, one can obtain

$$
G_{26} \leq\left(\left(\widehat{M}_{24}\right)^{(4)}\right)_{3}=G_{26}^{0}+2\left(a_{26}\right)^{(4)}\left(\left(\widehat{M}_{24}\right)^{(4)}\right)_{2} /\left(a_{26}^{\prime}\right)^{(4)}
$$

If $G_{25}$ or $G_{26}$ is bounded, the same property follows for $G_{24}, G_{26}$ and $G_{24}, G_{25}$ respectively.

Remark 4: If $G_{24}$ is bounded, from below, the same property holds for $G_{25}$ and $G_{26}$. The proof is 54 analogous with the preceding one. An analogous property is true if $G_{25}$ is bounded from below.

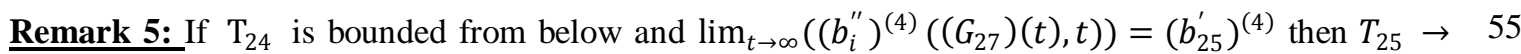
$\infty$.

Definition of $(m)^{(4)}$ and $\varepsilon_{4}$ :

Indeed let $t_{4}$ be so that for $t>t_{4}$

$$
\left(b_{25}\right)^{(4)}-\left(b_{i}^{\prime \prime}\right)^{(4)}\left(\left(G_{27}\right)(t), t\right)<\varepsilon_{4}, T_{24}(t)>(m)^{(4)}
$$

Then $\frac{d T_{25}}{d t} \geq\left(a_{25}\right)^{(4)}(m)^{(4)}-\varepsilon_{4} T_{25}$ which leads to

$T_{25} \geq\left(\frac{\left(a_{25}\right)^{(4)}(m)^{(4)}}{\varepsilon_{4}}\right)\left(1-e^{-\varepsilon_{4} t}\right)+T_{25}^{0} e^{-\varepsilon_{4} t}$ If we take $t$ such that $e^{-\varepsilon_{4} t}=\frac{1}{2}$ it results

$T_{25} \geq\left(\frac{\left(a_{25}\right)^{(4)}(m)(4)}{2}\right), \quad t=\log \frac{2}{\varepsilon_{4}}$ By taking now $\varepsilon_{4} \quad$ sufficiently small one sees that $\mathrm{T}_{25}$ is unbounded. The same property holds for $T_{26}$ if $\lim _{t \rightarrow \infty}\left(b_{26}^{\prime \prime}\right)^{(4)}\left(\left(G_{27}\right)(t), t\right)=\left(b_{26}^{\prime}\right)^{(4)}$

We now state a more precise theorem about the behaviors at infinity of the solutions of equations 37 to 42

\section{Behavior of the solutions of equation 37 to 12}

Theorem 2: If we denote and define 
Definition of $\left(\sigma_{1}\right)^{(4)},\left(\sigma_{2}\right)^{(4)},\left(\tau_{1}\right)^{(4)},\left(\tau_{2}\right)^{(4)}$ :

(a) $\left(\sigma_{1}\right)^{(4)},\left(\sigma_{2}\right)^{(4)},\left(\tau_{1}\right)^{(4)},\left(\tau_{2}\right)^{(4)}$ four constants satisfying

$$
\begin{gathered}
-\left(\sigma_{2}\right)^{(4)} \leq-\left(a_{24}^{\prime}\right)^{(4)}+\left(a_{25}^{\prime}\right)^{(4)}-\left(a_{24}^{\prime \prime}\right)^{(4)}\left(T_{25}, t\right)+\left(a_{25}^{\prime \prime}\right)^{(4)}\left(T_{25}, t\right) \leq-\left(\sigma_{1}\right)^{(4)} \\
-\left(\tau_{2}\right)^{(4)} \leq-\left(b_{24}^{\prime}\right)^{(4)}+\left(b_{25}^{\prime}\right)^{(4)}-\left(b_{24}^{\prime \prime}\right)^{(4)}\left(\left(G_{27}\right), t\right)-\left(b_{25}^{\prime \prime}\right)^{(4)}\left(\left(G_{27}\right), t\right) \leq-\left(\tau_{1}\right)^{(4)}
\end{gathered}
$$

Definition of $\left(v_{1}\right)^{(4)},\left(v_{2}\right)^{(4)},\left(u_{1}\right)^{(4)},\left(u_{2}\right)^{(4)}, v^{(4)}, u^{(4)}$ :

(b) By $\left(v_{1}\right)^{(4)}>0,\left(v_{2}\right)^{(4)}<0$ and respectively $\left(u_{1}\right)^{(4)}>0,\left(u_{2}\right)^{(4)}<0$ the roots of the 60 equations $\left(a_{25}\right)^{(4)}\left(v^{(4)}\right)^{2}+\left(\sigma_{1}\right)^{(4)} v^{(4)}-\left(a_{24}\right)^{(4)}=0$

and $\left(b_{25}\right)^{(4)}\left(u^{(4)}\right)^{2}+\left(\tau_{1}\right)^{(4)} u^{(4)}-\left(b_{24}\right)^{(4)}=0$ and

Definition of $\left(\bar{v}_{1}\right)^{(4)},\left(\bar{v}_{2}\right)^{(4)},\left(\bar{u}_{1}\right)^{(4)},\left(\bar{u}_{2}\right)^{(4)}$ :

By $\left(\bar{v}_{1}\right)^{(4)}>0,\left(\bar{v}_{2}\right)^{(4)}<0$ and respectively $\left(\bar{u}_{1}\right)^{(4)}>0,\left(\bar{u}_{2}\right)^{(4)}<0$ the

roots of the equations $\left(a_{25}\right)^{(4)}\left(v^{(4)}\right)^{2}+\left(\sigma_{2}\right)^{(4)} v^{(4)}-\left(a_{24}\right)^{(4)}=0$

and $\left(b_{25}\right)^{(4)}\left(u^{(4)}\right)^{2}+\left(\tau_{2}\right)^{(4)} u^{(4)}-\left(b_{24}\right)^{(4)}=0$

Definition of $\left(m_{1}\right)^{(4)},\left(m_{2}\right)^{(4)},\left(\mu_{1}\right)^{(4)},\left(\mu_{2}\right)^{(4)},\left(v_{0}\right)^{(4)}$ :-

(c) If we define $\left(m_{1}\right)^{(4)},\left(m_{2}\right)^{(4)},\left(\mu_{1}\right)^{(4)},\left(\mu_{2}\right)^{(4)}$ by

$$
\begin{gathered}
\left(m_{2}\right)^{(4)}=\left(v_{0}\right)^{(4)},\left(m_{1}\right)^{(4)}=\left(v_{1}\right)^{(4)}, \quad \text { if }\left(v_{0}\right)^{(4)}<\left(v_{1}\right)^{(4)} \\
\left(m_{2}\right)^{(4)}=\left(v_{1}\right)^{(4)},\left(m_{1}\right)^{(4)}=\left(\bar{v}_{1}\right)^{(4)}, \text { if }\left(v_{4}\right)^{(4)}<\left(v_{0}\right)^{(4)}<\left(\bar{v}_{1}\right)^{(4),}
\end{gathered}
$$

and $\left(v_{0}\right)^{(4)}=\frac{G_{24}^{0}}{G_{25}^{0}}$

$$
\left(m_{2}\right)^{(4)}=\left(v_{4}\right)^{(4)},\left(m_{1}\right)^{(4)}=\left(v_{0}\right)^{(4)}, \quad \text { if }\left(\bar{v}_{4}\right)^{(4)}<\left(v_{0}\right)^{(4)}
$$

and analogously

$$
\begin{gathered}
\left(\mu_{2}\right)^{(4)}=\left(u_{0}\right)^{(4)},\left(\mu_{1}\right)^{(4)}=\left(u_{1}\right)^{(4)}, \quad \text { if }\left(u_{0}\right)^{(4)}<\left(u_{1}\right)^{(4)} \\
\left(\mu_{2}\right)^{(4)}=\left(u_{1}\right)^{(4)},\left(\mu_{1}\right)^{(4)}=\left(\bar{u}_{1}\right)^{(4)}, \text { if }\left(u_{1}\right)^{(4)}<\left(u_{0}\right)^{(4)}<\left(\bar{u}_{1}\right)^{(4)},
\end{gathered}
$$

and $\left(u_{0}\right)^{(4)}=\frac{T_{24}^{0}}{T_{25}^{0}}$

$$
\left(\mu_{2}\right)^{(4)}=\left(u_{1}\right)^{(4)},\left(\mu_{1}\right)^{(4)}=\left(u_{0}\right)^{(4)}, \text { if }\left(\bar{u}_{1}\right)^{(4)}<\left(u_{0}\right)^{(4)} \text { where }\left(u_{1}\right)^{(4)},\left(\bar{u}_{1}\right)^{(4)}
$$

are defined by 59 and 64 respectively

Then the solution of 19,20,21,22,23 and 24 satisfies the inequalities

$$
G_{24}^{0} e^{\left(\left(S_{1}\right)^{(4)}-\left(p_{24}\right)^{(4)}\right) t} \leq G_{24}(t) \leq G_{24}^{0} e^{\left(S_{1}\right)^{(4)} t}
$$


where $\left(p_{i}\right)^{(4)}$ is defined by equation 25

$$
\begin{gathered}
\frac{1}{\left(m_{1}\right)^{(4)}} G_{24}^{0} e^{\left(\left(S_{1}\right)^{(4)}-\left(p_{24}\right)^{(4)}\right) t} \leq G_{25}(t) \leq \frac{1}{\left(m_{2}\right)^{(4)}} G_{24}^{0} e^{\left(S_{1}\right)^{(4)} t} \\
\left(\frac{\left(a_{26}\right)^{(4)} G_{24}^{0}}{\left(m_{1}\right)^{(4)}\left(\left(S_{1}\right)^{(4)}-\left(p_{24}\right)^{(4)}-\left(S_{2}\right)^{(4)}\right)}\left[e^{\left(\left(S_{1}\right)^{(4)}-\left(p_{24}\right)^{(4)}\right) t}-e^{-\left(S_{2}\right)^{(4)} t}\right]+G_{26}^{0} e^{-\left(S_{2}\right)^{(4)} t} \leq G_{26}(t)\right. \\
\left.\leq \frac{\left(a_{26}\right)^{(4)} G_{24}^{0}}{\left(m_{2}\right)^{(4)}\left(\left(S_{1}\right)^{(4)}-\left(a_{26}^{\prime}\right)^{(4)}\right)}\left[e^{\left(S_{1}\right)^{(4)} t}-e^{-\left(a_{26}^{\prime}\right)^{(4)} t}\right]+G_{26}^{0} e^{-\left(a_{26}^{\prime}\right)^{(4)} t}\right) \\
T_{24}^{0} e^{\left(R_{1}\right)^{(4)} t} \leq T_{24}(t) \leq T_{24}^{0} e^{\left(\left(R_{1}\right)^{(4)}+\left(r_{24}\right)^{(4)}\right) t} \\
\frac{1}{\left(\mu_{1}\right)^{(4)}} T_{24}^{0} e^{\left(R_{1}\right)^{(4)} t} \leq T_{24}(t) \leq \frac{1}{\left(\mu_{2}\right)^{(4)}} T_{24}^{0} e^{\left(\left(R_{1}\right)^{(4)}+\left(r_{24}\right)^{(4)}\right) t} \\
\frac{\left(b_{26}\right)^{(4)} T_{24}^{0}}{\left(\mu_{1}\right)^{(4)}\left(\left(R_{1}\right)^{(4)}-\left(b_{26}^{\prime}\right)^{(4)}\right)}\left[e^{\left(R_{1}\right)^{(4)} t}-e^{-\left(b_{26}^{\prime}\right)^{(4)} t}\right]+T_{26}^{0} e^{-\left(b_{26}^{\prime}\right)^{(4)} t} \leq T_{26}(t) \leq \\
\frac{\left(a_{26}\right)^{(4)} T_{24}^{0}}{\left(\mu_{2}\right)^{(4)}\left(\left(R_{1}\right)^{(4)}+\left(r_{24}\right)^{(4)}+\left(R_{2}\right)^{(4)}\right)}\left[e^{\left(\left(R_{1}\right)^{(4)}+\left(r_{24}\right)^{(4)}\right) t}-e^{-\left(R_{2}\right)^{(4)} t}\right]+T_{26}^{0} e^{-\left(R_{2}\right)^{(4)} t}
\end{gathered}
$$

Definition of $\left(S_{1}\right)^{(4)},\left(S_{2}\right)^{(4)},\left(R_{1}\right)^{(4)},\left(R_{2}\right)^{(4)}$ :-

Where $\left(S_{1}\right)^{(4)}=\left(a_{24}\right)^{(4)}\left(m_{2}\right)^{(4)}-\left(a_{24}^{\prime}\right)^{(4)}$

$$
\begin{gathered}
\left(S_{2}\right)^{(4)}=\left(a_{26}\right)^{(4)}-\left(p_{26}\right)^{(4)} \\
\left(R_{1}\right)^{(4)}=\left(b_{24}\right)^{(4)}\left(\mu_{2}\right)^{(4)}-\left(b_{24}^{\prime}\right)^{(4)} \\
\left(R_{2}\right)^{(4)}=\left(b_{26}^{\prime}\right)^{(4)}-\left(r_{26}\right)^{(4)}
\end{gathered}
$$

Proof : From 19,20,21,22,23,24 we obtain

$$
\frac{d v^{(4)}}{d t}=\left(a_{24}\right)^{(4)}-\left(\left(a_{24}^{\prime}\right)^{(4)}-\left(a_{25}^{\prime}\right)^{(4)}+\left(a_{24}^{\prime \prime}\right)^{(4)}\left(T_{25}, t\right)\right)-\left(a_{25}^{\prime \prime}\right)^{(4)}\left(T_{25}, t\right) v^{(4)}-\left(a_{25}\right)^{(4)} v^{(4)}
$$

Definition of $v^{(4)}:-\quad v^{(4)}=\frac{G_{24}}{G_{25}}$

It follows

$$
-\left(\left(a_{25}\right)^{(4)}\left(v^{(4)}\right)^{2}+\left(\sigma_{2}\right)^{(4)} v^{(4)}-\left(a_{24}\right)^{(4)}\right) \leq \frac{d v^{(4)}}{d t} \leq-\left(\left(a_{25}\right)^{(4)}\left(v^{(4)}\right)^{2}+\left(\sigma_{4}\right)^{(4)} v^{(4)}-\left(a_{24}\right)^{(4)}\right)
$$

From which one obtains

Definition of $\left(\bar{v}_{1}\right)^{(4)},\left(v_{0}\right)^{(4)}$ :-

(a) For $0<\left(v_{0}\right)^{(4)}=\frac{G_{24}^{0}}{G_{25}^{0}}<\left(v_{1}\right)^{(4)}<\left(\bar{v}_{1}\right)^{(4)}$ 


$$
\begin{gathered}
v^{(4)}(t) \geq \frac{\left(v_{1}\right)^{(4)}+(C)^{(4)}\left(v_{2}\right)^{(4)} e^{\left.\left[-\left(a_{25}\right)^{(4)}\left(v_{1}\right)^{(4)}-\left(v_{0}\right)^{(4)}\right) t\right]}}{1+(C)^{(4)} e^{\left.-\left(a_{25}\right)^{(4)}\left(\left(v_{1}\right)^{(4)}-\left(v_{0}\right)^{(4)}\right) t\right]}}, \quad(C)^{(4)}=\frac{\left(v_{1}\right)^{(4)}-\left(v_{0}\right)^{(4)}}{\left(v_{0}\right)^{(4)}-\left(v_{2}\right)^{(4)}} \\
\text { it follows }\left(v_{0}\right)^{(4)} \leq v^{(4)}(t) \leq\left(v_{1}\right)^{(4)}
\end{gathered}
$$

In the same manner, we get

$$
v^{(4)}(t) \leq \frac{\left(\bar{v}_{1}\right)^{(4)}+(\bar{C})^{(4)}\left(\bar{v}_{2}\right)^{(4)} e^{\left[-\left(a_{25}\right)^{(4)}\left(\left(\bar{v}_{1}\right)^{(4)}-\left(\bar{v}_{2}\right)^{(4)}\right) t\right]}}{1+(\bar{C})^{(4)} e^{\left.\left.-\left(a_{25}\right)^{(4)}\left(\bar{v}_{1}\right)^{(4)}-\left(\bar{v}_{2}\right)^{(4)}\right) t\right]}} \quad, \quad(\bar{C})^{(4)}=\frac{\left(\bar{v}_{1}\right)^{(4)}-\left(v_{0}\right)^{(4)}}{\left(v_{0}\right)^{(4)}-\left(\bar{v}_{2}\right)^{(4)}}
$$

From which we deduce $\left(v_{0}\right)^{(4)} \leq v^{(4)}(t) \leq\left(\bar{v}_{1}\right)^{(4)}$

(b) If $0<\left(v_{1}\right)^{(4)}<\left(v_{0}\right)^{(4)}=\frac{G_{24}^{0}}{G_{25}^{0}}<\left(\bar{v}_{1}\right)^{(4)}$ we find like in the previous case,

$$
\begin{gathered}
\left(v_{1}\right)^{(4)} \leq \frac{\left(v_{1}\right)^{(4)}+(C)^{(4)}\left(v_{2}\right)^{(4)} e^{\left[-\left(a_{25}\right)^{(4)}\left(\left(v_{1}\right)^{(4)}-\left(v_{2}\right)^{(4)}\right) t\right]}}{1+(C)^{(4)} e^{\left[-\left(a_{25}\right)^{(4)}\left(\left(v_{1}\right)^{(4)}-\left(v_{2}\right)^{(4)}\right) t\right]}} \leq v^{(4)}(t) \leq \\
\frac{\left(\bar{v}_{1}\right)^{(4)}+(\bar{C})^{(4)}\left(\bar{v}_{2}\right)^{(4)} e^{\left.\left[-\left(a_{25}\right)^{(4)}\left(\bar{v}_{1}\right)^{(4)}-\left(\bar{v}_{2}\right)^{(4)}\right) t\right]}}{1+(\bar{C})^{(4)} e^{\left.\left[-\left(a_{25}\right)^{(4)}\left(\bar{v}_{1}\right)^{(4)}-\left(\bar{v}_{2}\right)^{(4)}\right) t\right]}} \leq\left(\bar{v}_{1}\right)^{(4)}
\end{gathered}
$$

(c) If $0<\left(v_{1}\right)^{(4)} \leq\left(\bar{v}_{1}\right)^{(4)} \leq\left(v_{0}\right)^{(4)}=\frac{G_{24}^{0}}{G_{25}^{0}}$, we obtain

$$
\left(v_{1}\right)^{(4)} \leq v^{(4)}(t) \leq \frac{\left(\bar{v}_{1}\right)^{(4)}+(\bar{C})^{(4)}\left(\bar{v}_{2}\right)^{(4)} e^{\left.\left[-\left(a_{25}\right)^{(4)}\left(\bar{v}_{1}\right)^{(4)}-\left(\bar{v}_{2}\right)^{(4)}\right) t\right]}}{1+(\bar{C})^{(4)} e^{\left.\left[-\left(a_{25}\right)^{(4)}\left(\bar{v}_{1}\right)^{(4)}-\left(\bar{v}_{2}\right)^{(4)}\right) t\right]}} \leq\left(v_{0}\right)^{(4)}
$$

And so with the notation of the first part of condition (c), we have

Definition of $v^{(4)}(t)$ :-

$$
\left(m_{2}\right)^{(4)} \leq v^{(4)}(t) \leq\left(m_{1}\right)^{(4)}, v^{(4)}(t)=\frac{G_{24}(t)}{G_{25}(t)}
$$

In a completely analogous way, we obtain

Definition of $u^{(4)}(t)$ :-

$\left(\mu_{2}\right)^{(4)} \leq u^{(4)}(t) \leq\left(\mu_{1}\right)^{(4)}, u^{(4)}(t)=\frac{T_{24}(t)}{T_{25}(t)}$

Now, using this result and replacing it in 19, 20,21,22,23, and 24 we get easily the result stated in the theorem.

\section{Particular case :}

If $\left(a_{24}^{\prime \prime}\right)^{(4)}=\left(a_{25}^{\prime \prime}\right)^{(4)}$, then $\left(\sigma_{1}\right)^{(4)}=\left(\sigma_{2}\right)^{(4)}$ and in this case $\left(v_{1}\right)^{(4)}=\left(\bar{v}_{1}\right)^{(4)}$ if in addition $\left(v_{0}\right)^{(4)}=\left(v_{1}\right)^{(4)}$ then $v^{(4)}(t)=\left(v_{0}\right)^{(4)}$ and as a consequence $G_{24}(t)=\left(v_{0}\right)^{(4)} G_{25}(t)$ this also defines $\left(v_{0}\right)^{(4)}$ for the special case .

Analogously if $\left(b_{24}^{\prime \prime}\right)^{(4)}=\left(b_{25}^{\prime \prime}\right)^{(4)}$, then $\left(\tau_{1}\right)^{(4)}=\left(\tau_{2}\right)^{(4)}$ and then $\left(u_{1}\right)^{(4)}=\left(\bar{u}_{4}\right)^{(4)}$ if in addition $\left(u_{0}\right)^{(4)}=\left(u_{1}\right)^{(4)}$ then $T_{24}(t)=\left(u_{0}\right)^{(4)} T_{25}(t)$ This is an important www.iosrjournals.org 
consequence of the relation between $\left(v_{1}\right)^{(4)}$ and $\left(\bar{v}_{1}\right)^{(4)}$, and definition of $\left(u_{0}\right)^{(4)}$.

\section{STATIONARY SOLUTIONS AND STABILITY}

Stationary solutions and stability curve representative of the variation of VE-QF SYATEM curve lies below the tangent at $\left(G_{27}\right)=G_{0}$ for $\left(G_{27}\right)<G_{0}$ and above the tangent for $\left(G_{27}\right)>G_{0}$ .Wherever such a situation occurs the point $G_{0}$ is called the "point of inflexion". In this case, the tangent has a positive slope that simply means the rate of change of VE is greater than zero. Above factor shows that it is possible, to draw a curve that has a point of inflexion at a point where the tangent (slope of the curve) is horizontal.

\section{Stationary value :}

In all the cases $\left(G_{27}\right)=G_{0},\left(G_{27}\right)<G_{0},\left(G_{27}\right)>G_{0}$ the condition that the rate of change of oxygen consumption is maximum or minimum holds. When this condition holds we have stationary value. We now infer that :

1. A necessary and sufficient condition for there to be stationary value of $\left(G_{27}\right)$ is that the rate of change of VE function at $G_{0}$ is zero.

2. A sufficient condition for the stationary value at $G_{0}$, to be maximum is that the acceleration of the VE is less than zero.

3. A sufficient condition for the stationary value at $G_{0}$, be minimum is that acceleration of VE is greater than zero.

4. With the rate of change of $\left(G_{27}\right)\left(T_{27}\right)$ defined as the accentuation term and the dissipation term, we are sure that the rate of change of VE $\mathrm{n}$ is always positive.

5. Concept of stationary state is mere methodology although there might be closed system exhibiting symptoms of stationariness.

We can prove the following

Theorem 3: If $\left(a_{i}^{\prime \prime}\right)^{(4)}$ and $\left(b_{i}^{\prime \prime}\right)^{(4)}$ are independent on $t$, and the conditions (with the notations $25,26,27,28$ )

$$
\begin{gathered}
\left(a_{24}^{\prime}\right)^{(4)}\left(a_{25}^{\prime}\right)^{(4)}-\left(a_{24}\right)^{(4)}\left(a_{25}\right)^{(4)}<0 \\
\left(a_{24}^{\prime}\right)^{(4)}\left(a_{25}^{\prime}\right)^{(4)}-\left(a_{24}\right)^{(4)}\left(a_{25}\right)^{(4)}+\left(a_{24}\right)^{(4)}\left(p_{24}\right)^{(4)}+\left(a_{25}^{\prime}\right)^{(4)}\left(p_{25}\right)^{(4)}+\left(p_{24}\right)^{(4)}\left(p_{25}\right)^{(4)}>0 \\
\left(b_{24}^{\prime}\right)^{(4)}\left(b_{25}^{\prime}\right)^{(4)}-\left(b_{24}\right)^{(4)}\left(b_{25}\right)^{(4)}>0, \\
\left(b_{24}^{\prime}\right)^{(4)}\left(b_{25}^{\prime}\right)^{(4)}-\left(b_{24}\right)^{(4)}\left(b_{25}\right)^{(4)}-\left(b_{24}^{\prime}\right)^{(4)}\left(r_{25}\right)^{(4)}-\left(b_{25}^{\prime}\right)^{(4)}\left(r_{25}\right)^{(4)}+\left(r_{24}\right)^{(4)}\left(r_{25}\right)^{(4)}<0
\end{gathered}
$$

with $\left(p_{24}\right)^{(4)},\left(r_{25}\right)^{(4)}$ as defined by equation 25 are satisfied, then the system

$$
\begin{aligned}
& \left(a_{24}\right)^{(4)} G_{25}-\left[\left(a_{24}^{\prime}\right)^{(4)}+\left(a_{24}^{\prime \prime}\right)^{(4)}\left(T_{25}\right)\right] G_{24}=0 \\
& \left(a_{25}\right)^{(4)} G_{24}-\left[\left(a_{25}^{\prime}\right)^{(4)}+\left(a_{25}^{\prime \prime}\right)^{(4)}\left(T_{25}\right)\right] G_{25}=0 \\
& \left(a_{26}\right)^{(4)} G_{25}-\left[\left(a_{26}^{\prime}\right)^{(4)}+\left(a_{26}^{\prime \prime}\right)^{(4)}\left(T_{25}\right)\right] G_{26}=0 \\
& \left(b_{24}\right)^{(4)} T_{25}-\left[\left(b_{24}^{\prime}\right)^{(4)}-\left(b_{24}^{\prime \prime}\right)^{(4)}\left(\left(G_{27}\right)\right)\right] T_{24}=0 \\
& \left(b_{25}\right)^{(4)} T_{24}-\left[\left(b_{25}^{\prime}\right)^{(4)}-\left(b_{25}^{\prime \prime}\right)^{(4)}\left(\left(G_{27}\right)\right)\right] T_{25}=0
\end{aligned}
$$




$$
\left(b_{26}\right)^{(4)} T_{25}-\left[\left(b_{26}^{\prime}\right)^{(4)}-\left(b_{26}^{\prime \prime}\right)^{(4)}\left(\left(G_{27}\right)\right)\right] T_{26}=0
$$

has a unique positive solution, which is an equilibrium solution for the system (19 to 24)

\section{Proof:}

(a) Indeed the first two equations have a nontrivial solution $G_{24}, G_{25}$ if

$$
\begin{aligned}
& F\left(T_{27}\right)=\left(a_{24}^{\prime}\right)^{(4)}\left(a_{25}^{\prime}\right)^{(4)}-\left(a_{24}\right)^{(4)}\left(a_{25}\right)^{(4)}+\left(a_{24}^{\prime}\right)^{(4)}\left(a_{25}^{\prime \prime}\right)^{(4)}\left(T_{25}\right) \\
& +\left(a_{25}^{\prime}\right)^{(4)}\left(a_{24}^{\prime \prime}\right)^{(4)}\left(T_{25}\right)+\left(a_{24}^{\prime \prime}\right)^{(4)}\left(T_{25}\right)\left(a_{25}^{\prime \prime}\right)^{(4)}\left(T_{25}\right)=0
\end{aligned}
$$

\section{Definition and uniqueness of $\mathrm{T}_{25}^{*}$ :-}

After hypothesis $f(0)<0, f(\infty)>0$ and the functions $\left(a_{i}^{\prime \prime}\right)^{(4)}\left(T_{25}\right)$ being increasing, it follows that there exists a unique $T_{25}^{*}$ for which $f\left(T_{25}^{*}\right)=0$. With this value, we obtain from the three first equations

$G_{24}=\frac{\left(a_{24}\right)^{(4)} G_{25}}{\left[\left(a_{24}^{\prime}\right)^{(4)}+\left(a_{24}^{\prime \prime}\right)^{(4)}\left(T_{25}^{*}\right)\right]} \quad, \quad G_{26}=\frac{\left(a_{26}\right)^{(4)} G_{25}}{\left[\left(a_{26}^{\prime}\right)^{(4)}+\left(a_{26}^{\prime \prime}\right)^{(4)}\left(T_{25}^{*}\right)\right]}$

(b) By the same argument, the equations 92,93 admit solutions $G_{24}, G_{25}$ if

$$
\begin{gathered}
\varphi\left(G_{27}\right)=\left(b_{24}^{\prime}\right)^{(4)}\left(b_{25}^{\prime}\right)^{(4)}-\left(b_{24}\right)^{(4)}\left(b_{25}\right)^{(4)}- \\
{\left[\left(b_{24}^{\prime}\right)^{(4)}\left(b_{25}^{\prime \prime}\right)^{(4)}\left(G_{27}\right)+\left(b_{25}^{\prime}\right)^{(4)}\left(b_{24}^{\prime \prime}\right)^{(4)}\left(G_{27}\right)\right]+\left(b_{24}^{\prime \prime}\right)^{(4)}\left(G_{27}\right)\left(b_{25}^{\prime \prime}\right)^{(4)}\left(G_{27}\right)=0}
\end{gathered}
$$

Where in $\left(G_{27}\right)\left(G_{24}, G_{25}, G_{26}\right), G_{24}, G_{26}$ must be replaced by their values from 96 . It is easy to see that $\varphi$ is a decreasing function in $G_{25}$ taking into account the hypothesis $\varphi(0)>0, \varphi(\infty)<0$ it follows that there exists a unique $G_{25}^{*}$ such that $\varphi\left(\left(G_{27}\right)^{*}\right)=0$

Finally we obtain the unique solution of 89 to 94

$G_{25}^{*}$ given by $\varphi\left(\left(G_{27}\right)^{*} G^{*}\right)=0, T_{25}^{*}$ given by $f\left(T_{25}^{*}\right)=0$ and

$G_{24}^{*}=\frac{\left(a_{24}\right)^{(4)} G_{25}^{*}}{\left[\left(a_{24}^{\prime}\right)^{(4)}+\left(a_{24}^{\prime \prime}\right)^{(4)}\left(T_{25}^{*}\right)\right]} \quad, \quad G_{26}^{*}=\frac{\left(a_{26}\right)^{(4)} G_{25}^{*}}{\left[\left(a_{26}^{\prime}\right)^{(4)}+\left(a_{26}^{\prime \prime}\right)^{(4)}\left(T_{25}^{*}\right)\right]}$

$T_{24}^{*}=\frac{\left(b_{24}\right)^{(4)} T_{25}^{*}}{\left[\left(b_{24}^{\prime}\right)^{(4)}-\left(b_{24}^{\prime \prime}\right)^{(4)}\left(\left(G_{27}\right)^{*}\right)\right]} \quad, \quad T_{26}^{*}=\frac{\left(b_{26}\right)^{(4)} T_{25}^{*}}{\left[\left(b_{26}^{\prime}\right)^{(4)}-\left(b_{26}^{\prime \prime}\right)^{(4)}\left(\left(G_{27}\right)^{*}\right)\right]}$

Obviously, these values represent an equilibrium solution of 19,20,21,22,23,24

\section{ASYMPTOTIC STABILITY ANALYSIS}

Theorem 4: If the conditions of the previous theorem are satisfied and if the functions $\left(a_{i}^{\prime \prime}\right)^{(4)}$ and $\left(b_{i}^{\prime \prime}\right)^{(4)}$ Belong to $C^{(4)}\left(\mathbb{R}_{+}\right)$then the above equilibrium point is asymptotically stable.

Proof: Denote

Definition of $\mathbb{G}_{i}, \mathbb{T}_{i}:-$

$$
G_{i}=G_{i}^{*}+\mathbb{G}_{i} \quad, T_{i}=T_{i}^{*}+\mathbb{T}_{i}
$$




$$
\frac{\partial\left(a_{25}^{\prime \prime}\right)^{(4)}}{\partial T_{25}}\left(T_{25}^{*}\right)=\left(q_{25}\right)^{(4)}, \frac{\partial\left(b_{i}^{\prime \prime}\right)^{(4)}}{\partial G_{j}}\left(\left(G_{27}\right)^{*}\right)=s_{i j}
$$

Then taking into account equations 89 to 94 and neglecting the terms of power 2, we obtain from 49 to 24

$$
\begin{aligned}
& \frac{d \mathbb{G}_{24}}{d t}=-\left(\left(a_{24}^{\prime}\right)^{(4)}+\left(p_{24}\right)^{(4)}\right) \mathbb{G}_{24}+\left(a_{24}\right)^{(4)} \mathbb{G}_{25}-\left(q_{24}\right)^{(4)} G_{24}^{*} \mathbb{T}_{25} \\
& \frac{d \mathbb{G}_{25}}{d t}=-\left(\left(a_{25}^{\prime}\right)^{(4)}+\left(p_{25}\right)^{(4)}\right) \mathbb{G}_{25}+\left(a_{25}\right)^{(4)} \mathbb{G}_{24}-\left(q_{25}\right)^{(4)} G_{25}^{*} \mathbb{T}_{25} \\
& \frac{d \mathbb{G}_{26}}{d t}=-\left(\left(a_{26}^{\prime}\right)^{(4)}+\left(p_{26}\right)^{(4)}\right) \mathbb{G}_{26}+\left(a_{26}\right)^{(4)} \mathbb{G}_{25}-\left(q_{26}\right)^{(4)} G_{26}^{*} \mathbb{T}_{25} \\
& \frac{d \mathbb{T}_{24}}{d t}=-\left(\left(b_{24}^{\prime}\right)^{(4)}-\left(r_{24}\right)^{(4)}\right) \mathbb{T}_{24}+\left(b_{24}\right)^{(4)} \mathbb{T}_{25}+\sum_{j=24}^{26}\left(s_{(24)(j)} T_{24}^{*} \mathbb{G}_{j}\right) \\
& \frac{d \mathbb{T}_{25}}{d t}=-\left(\left(b_{25}^{\prime}\right)^{(4)}-\left(r_{25}\right)^{(4)}\right) \mathbb{T}_{25}+\left(b_{25}\right)^{(4)} \mathbb{T}_{24}+\sum_{j=24}^{26}\left(s_{(25)(j)} T_{25}^{*} \mathbb{G}_{j}\right) \\
& \frac{d \mathbb{T}_{26}}{d t}=-\left(\left(b_{26}^{\prime}\right)^{(4)}-\left(r_{26}\right)^{(4)}\right) \mathbb{T}_{26}+\left(b_{26}\right)^{(4)} \mathbb{T}_{25}+\sum_{j=24}^{26}\left(s_{(26)(j)} T_{26}^{*} \mathbb{G}_{j}\right)
\end{aligned}
$$

The characteristic equation of this system is

$$
\begin{aligned}
& \left((\lambda)^{(4)}+\left(b_{26}^{\prime}\right)^{(4)}-\left(r_{26}\right)^{(4)}\right)\left\{\left((\lambda)^{(4)}+\left(a_{26}^{\prime}\right)^{(4)}+\left(p_{26}\right)^{(4)}\right)\right. \\
& {\left[\left(\left((\lambda)^{(4)}+\left(a_{24}^{\prime}\right)^{(4)}+\left(p_{24}\right)^{(4)}\right)\left(q_{25}\right)^{(4)} G_{25}^{*}+\left(a_{25}\right)^{(4)}\left(q_{24}\right)^{(4)} G_{24}^{*}\right)\right]} \\
& \left(\left((\lambda)^{(4)}+\left(b_{24}^{\prime}\right)^{(4)}-\left(r_{24}\right)^{(4)}\right) s_{(25),(25)} T_{25}^{*}+\left(b_{25}\right)^{(4)} s_{(24),(25)} T_{25}^{*}\right) \\
& +\left(\left((\lambda)^{(4)}+\left(a_{25}^{\prime}\right)^{(4)}+\left(p_{25}\right)^{(4)}\right)\left(q_{24}\right)^{(4)} G_{24}^{*}+\left(a_{24}\right)^{(4)}\left(q_{25}\right)^{(4)} G_{25}^{*}\right) \\
& \left(\left((\lambda)^{(4)}+\left(b_{24}^{\prime}\right)^{(4)}-\left(r_{24}\right)^{(4)}\right) s_{(25),(24)} T_{25}^{*}+\left(b_{25}\right)^{(4)} s_{(24),(24)} T_{24}^{*}\right) \\
& \left(\left((\lambda)^{(4)}\right)^{2}+\left(\left(a_{24}^{\prime}\right)^{(4)}+\left(a_{25}^{\prime}\right)^{(4)}+\left(p_{24}\right)^{(4)}+\left(p_{25}\right)^{(4)}\right)(\lambda)^{(4)}\right) \\
& \left(\left((\lambda)^{(4)}\right)^{2}+\left(\left(b_{24}^{\prime}\right)^{(4)}+\left(b_{25}^{\prime}\right)^{(4)}-\left(r_{24}\right)^{(4)}+\left(r_{25}\right)^{(4)}\right)(\lambda)^{(4)}\right) \\
& +\left(\left((\lambda)^{(4)}\right)^{2}+\left(\left(a_{24}^{\prime}\right)^{(4)}+\left(a_{25}^{\prime}\right)^{(4)}+\left(p_{24}\right)^{(4)}+\left(p_{25}\right)^{(4)}\right)(\lambda)^{(4)}\right)\left(q_{26}\right)^{(4)} G_{26} \\
& +\left((\lambda)^{(4)}+\left(a_{24}^{\prime}\right)^{(4)}+\left(p_{24}\right)^{(4)}\right)\left(\left(a_{26}\right)^{(4)}\left(q_{25}\right)^{(4)} G_{25}^{*}+\left(a_{25}\right)^{(4)}\left(a_{26}\right)^{(4)}\left(q_{24}\right)^{(4)} G_{24}^{*}\right) \\
& \left.\left(\left((\lambda)^{(4)}+\left(b_{24}^{\prime}\right)^{(4)}-\left(r_{24}\right)^{(4)}\right) s_{(25),(26)} T_{25}^{*}+\left(b_{25}\right)^{(4)} s_{(24),(26)} T_{24}^{*}\right)\right\}=0
\end{aligned}
$$

And as one sees, all the coefficients are positive. It follows that all the roots have negative real part, and this proves the theorem. 


\section{AN EPILOGUE ON VACUUM ENERGY,QUANTUM FIELD,ZERO POINT ENERGY,DARK MATTER AND THEIR INTERRELATIONSHIPS AND RAMIFICATIONS OF RESULTS OBTAINED ON THEM:}

Current models of the universe assume that is close to one. For example, in the inflationary theory a truly stupendous expansion is assumed to have occurred early in the history of the universe. About $10^{-34}$ seconds after the creation, when the temperature was perhaps one thousand trillion degrees, a bubble no more than $10^{-24} \mathrm{~cm}$ across might have undergone an expansion by a factor of maybe $10^{50}$ ! At about $10^{-}$ ${ }^{32}$ seconds this expansion stopped, leaving the bubble $10^{26} \mathrm{~cm}$ across (that is, 100 million light years). However, the bit of the universe we can observe would have been, after inflation, only a few centimeters across After $10^{-32}$ seconds, or so, the normal big bang evolution takes over. The fantastic stretching of space during inflation renders the observable universe geometrically flat.This is because the observable universe would be but a tiny patch of a universe that may be a trillion trillion times larger. Even if the geometry of the universe were initially highly curved the observable part of it is such a small patch that it would nonetheless appear to us as spatially flat, just as a small patch of the Earth's surface appears flat. Moreover, any weird particles or spacetime distortions that may have existed when the universe came into being would have been diluted far out of sight; we would not expect to find any such oddities in our neck of the woods. Lastly, the microwave background radiation, which was already uniform and isotropic within the initial bubble, would have remained so after inflation, which explains why the radiation at opposite sides of the sky has the same temperature; these patches of sky were once in casual contact (much less than $10^{-24} \mathrm{~cm}$ apart) but were then pushed far apart by inflation.

\section{DARK ENERGY AND VACUUM ENERGY}

Alas, $\Omega$ is observed to be much less than unity Therefore, if we nonetheless insist that $\Omega=1 \square$ we is forced to conclude that the luminous matter we observe forms only a part of the total energy in the universe. The deficit must therefore be made up by some other unknown source of energy. This could be dark matter. Much more interesting and much more mysterious: the possible existence of vacuum energy; or perhaps an admixture of the two.

Within the context of a general relativistic description of the universe there is two critical parameters that determine the global properties of the universe: The Hubble constant $\mathrm{H}_{0}$ and the deceleration parameter $\mathrm{q}_{0}$. If the universe is expanding today, it must have been expanding faster in the past provided that it is filled with normal matter and energy. This is because the gravitational force of normal matter is always attractive and therefore slows down the expansion. The deceleration parameter, as the name suggests, is a measure of how much the expansion has slowed since the big bang. We have already encountered Hubble's law

$\mathrm{Z}=\mathrm{d} \mathrm{H} \mathrm{H}_{0} / \mathrm{c}$,

Where $\mathrm{Z}$ is the redshift of a galaxy and $\mathrm{d}$ its (proper) distance from us. This law is valid only for small redshifts, say less than about 0.2 ; that is, for galaxies that are not too distant. For very distant galaxies we need a more accurate formula. We need this because when we look at very distant galaxies we are looking into the distant past when the expansion rate was, presumably, greater. A more accurate formula that takes account of the universal deceleration is

$\mathrm{Z}=\mathrm{d} \mathrm{H}_{0} / \mathrm{c}+\left(1+\mathrm{q}_{0}\right)\left(\mathrm{d} \mathrm{H}_{0} / \mathrm{c}\right)^{2} / 2$.

In practice, cosmologists do not use the proper distance $\mathrm{d}$, directly, but rather use a distance scale, called the luminosity distance, $d_{L}$, which is determined by requiring that the inverse square law

$f=L / 4 \pi d_{L}^{2}$

hold true even for an expanding universe. (In a static, flat universe both distance scales coincide.) Here $\mathrm{f}$ is the measured flux (energy per square meter per second) and $\mathrm{L}$ is the absolute luminosity (energy per second). 
The luminosity distance is convenient because it can be determined from measuring $\mathrm{f}$ and determining $\mathrm{L}$, only.

\section{MODEL THEORYAND LIMIT OF FINITE LINEAR ORDERINGS TO EXPLAIN THE STRUCTURE OF VACUUM ENERGY AND QUANTUM FIELD:}

In model theory, the age of a structure (or model) $A$ is the class of all finitely generated structures which are embeddable in $A$ (i.e. isomorphic to substructures of $A$ ). This concept is central in the construction of a Fraïssé limit. The main point of Fraïssé's construction is to show how one can approximate a structure by its finitely generated substructures. Thus for example the age of any dense linear order without endpoints (DLO), $\langle\mathbb{Q},\langle\rangle$ is precisely the set of all finite linear orderings, which are distinguished up to isomorphism only by their size. Thus the age of any DLO is countable. This shows in a way that a DLO is a kind of limit of finite linear orderings.

\section{Amalgamation property}

For any structures $\mathrm{A}, \mathrm{B}$ and $\mathrm{C}$ in $\mathrm{K}$ such that $\mathrm{A}$ is embeddable in both $\mathrm{B}$ and $\mathrm{C}$, there exists $\mathrm{D}$ in $\mathrm{K}$ to which $\mathrm{B}$ and $\mathrm{C}$ are both embeddable by embeddings which coincide on the image of $\mathrm{A}$ in both structures. In that case, there is a unique countable structure, up to isomorphism, that has age $\mathrm{K}$ and is homogeneous. In this context, Homogeneous means that any isomorphism between two finitely generated substructures can be extended to an automorphism of the whole structure. Again, an example of this situation is the ordered set of rational numbers $\langle\mathbb{Q},\langle\rangle$. It is the unique (up to isomorphism) homogenous countable structure whose age is the set of all finite linear orderings. Note that the ordered set of natural numbers $\langle\mathbb{N},\langle\rangle$ has the same age as a DLO, but it is not homogenous since if we map $\{1,3\}$ to $\{5,6\}$, it would not extend to any automorphism $\mathrm{f}$ since there should be an element between $f(\mathbf{1})=5$ and $f(3)=6$. The same applies to integers.

\section{ANTHROPIC PRINCIPLE-EVERYTHING HAS AGE - VACUUM ENERGY AND QUANTUM FIELD IN COSMOS HAS AGE}

In astrophysics and cosmology, the anthropic principle is the consideration that observations of the physical Universe must be compatible with the conscious life that observes it. Some proponents of the anthropic principle reason that it explains why the Universe has the age and the fundamental physical constants necessary to accommodate conscious life. As a result, they believe that the fact is unremarkable that the universe's fundamental constants happen to fall within the narrow range thought to be compatible with life. The strong anthropic principle (SAP) as explained by Barrow and Tipler (see variants) states that this is all the case because the Universe is compelled, in some sense, for conscious life to eventually emerge. Douglas Adams used the metaphor of a living puddle examining its own shape, since, to those living creatures, the universe may appear to fit them perfectly (while in fact, they simply fit the universe perfectly). Critics of the SAP argue in favor of a weak anthropic principle (WAP) similar to the one defined by Brandon Carter, which states that the universe's ostensible fine tuning is the result of selection bias: i.e., only in a universe capable of eventually supporting life will there be living beings capable of observing any such fine tuning, while a universe less compatible with life will go unbeheld.

The principle was formulated as a response to a series of observations that the laws of nature and parameters of the Universe take on values that are consistent with conditions for life as we know it rather than a set of values that would not be consistent with life on Earth. The anthropic principle states that this is a necessity, because if life were impossible, no one would know it. That is, it must be possible to observe some Universe, and hence, the laws and constants of any such universe must accommodate that possibility. The term anthropic in "anthropic principle" has been argued to be a misnomer, an anachronism both in predicational anteriority and character constitution.. Anthropic principle is a tautology or truism. In 1961, Robert Dicke noted that the age of the universe, as seen by living observers, cannot be random. Instead, 
biological factors constrain the universe to be more or less in a "golden age," neither too young nor too old. If the universe were one tenth as old as its present age, there would not have been sufficient time to build up appreciable levels of metallicity (levels of elements besides hydrogen and helium) especially carbon, by nucleosynthesis. Small rocky planets did not yet exist. If the universe were 10 times older than it actually is, most stars would be too old to remain on the main sequence and would have turned into white dwarfs, aside from the dimmest red dwarfs, and stable planetary systems would have already come to an end. Thus Dicke explained away the rough coincidence between large dimensionless numbers constructed from the constants of physics and the age of the universe, a coincidence which had inspired Dirac's varying-G theory.

Dicke later reasoned that the density of matter in the universe must be almost exactly the same as the critical density needed to prevent the Big Crunch (the "Dicke coincidences" argument). The most recent measurements may suggest that the observed density of baryonic matter, and some theoretical predictions of the amount of dark matter account for about $30 \%$ of this critical density, with the rest contributed by a cosmological constant. Steven Weinberg gave an anthropic explanation for this fact: he noted that the cosmological constant has a remarkably low value, some 120 orders of magnitude smaller than the value particle physics predicts. If the cosmological constant were more than about 10 times its observed value, then, it shall produce universe that would suffer catastrophic inflation, which would preclude the formation of stars, and hence life.

The observed values of the dimensionless physical constants (such as the fine-structure constant) governing the four fundamental interactions are balanced as if fine-tuned to permit the formation of commonly found matter and subsequently the emergence of life. A slight increase in the strong nuclear force would bind the dineutron and the diproton, and nuclear fusion would have converted all hydrogen in the early universe to helium. Water and the long-lived stable stars essential for the emergence of life as we know it would not exist. More generally, small changes in the relative strengths of the four fundamental interactions can greatly affect the universe's age, structure, and capacity for life. Carter defined two forms of the Anthropic Principle, a "weak" one which referred only to anthropic selection of privileged spacetime locations in the universe, and a more controversial "strong" form which addressed the values of the fundamental constants of physics.Roger Penrose explained the weak form as follows:

"The argument of weak anthropic principle can be used to explain why the conditions happen to be just right for the existence of (intelligent) life on the earth at the present time. For if they were not just right, then we should not have found ourselves to be here now, but somewhere else, at some other appropriate time. This principle was used very effectively by Brandon Carter and Robert Dicke to resolve an issue that had puzzled physicists for a good many years. The issue concerned various striking numerical relations that are observed to hold between the physical constants (the gravitational constant, the mass of the proton, the age of the universe, etc.). A puzzling aspect of this was that some of the relations hold only at the present epoch in the earth's history, so we appear, coincidentally, to be living at a very special time (give or take a few million years). This was later explained, by Carter and Dicke, by the fact that this epoch coincided with the lifetime of what are called main-sequence stars, such as the sun. At any other epoch, so the argument ran, there would be no intelligent life around in order to measure the physical constants in question - so the coincidence had to hold, simply because there would be intelligent life around only at the particular time that the coincidence did hold!"(-The Emperor's New Mind, Chapter 10)

First, we can use our own existence to make "predictions" about the parameters. But second, "as a last resort", we can convert these predictions into explanations by assuming that there is more than one Universe, in fact a large and possibly infinite collection of universes, something that is now called a multiverse ("world ensemble" was Carter's term), in which the parameters (and perhaps the laws of physics) vary across universes. The strong principle then becomes an example of a selection effect, exactly analogous to the weak principle. Postulating a multiverse is certainly a radical step, but taking it could provide at least a partial answer to a question which had seemed to be out of the reach of normal science: "why do the fundamental laws of physics take the particular form we observe and not another?"(See authors 
'Measurement disturbs Quantum Mechanical Explanation), This apparantely mean that perception changes reality. Perception is not reality.

Carter was not the first to invoke some form of the anthropic principle. In fact, the evolutionary biologist Alfred Russel Wallace anticipated the anthropic principle as long ago as 1904: "Such a vast and complex universe as that which we know exists around us, may have been absolutely required ... in order to produce a world that should be precisely adapted in every detail for the orderly development of life culminating in man." In 1957, Robert Dicke wrote: "The age of the Universe 'now' is not random but conditioned by biological factors ... changes in the values of the fundamental constants of physics] would preclude the existence of man to consider the problem. And how is it every things that happens could be understood in mathematical terms? Somebody knew that we are coming and also possess knowledge of Mathematics!

\section{STRONG AND WEAK ANTHROPIC PRINCIPLE:}

Strong anthropic principle (SAP) (Barrow and Tipler): "The Universe must have those properties which allow life to develop within it at some stage in its history. This looks very similar to Carter's SAP, but unlike the case with Carter's SAP, the "must" is an imperative, as shown by the following three possible elaborations of the SAP, each proposed by Barrow and Tipler"There exists one possible Universe 'designed' with the goal of generating and sustaining 'observers.'

This can be seen as simply the classic design argument(intelligent grand design) restated in the garb of contemporary cosmology. It implies that the purpose of the universe is to give rise to intelligent life, with the laws of nature and their fundamental physical constants set to ensure that life as we know it will emerge and evolve." Observers are necessary to bring the Universe into being."Presence of observers is necessary the existence of space time continuum. Perception of observers is essential functional prerequisite for the existence of the Universe. "An ensemble of other different universes is necessary for the existence of our Universe. "

Although philosophers have discussed related concepts for centuries, in the early 1970s the only genuine physical theory yielding a multiverse of sorts was the many worlds interpretation of quantum mechanics. This would allow variation in initial conditions, but not in the truly fundamental constants. Since that time a number of mechanisms for producing a multiverse have been suggested: (see the review by Max Tegmark). An important development in the 1980s was the combination of inflation theory with the hypothesis that some parameters are determined by symmetry breaking in the early universe, which allows parameters previously thought of as "fundamental constants" to vary over very large distances, thus eroding the distinction between Carter's weak and strong principles. At the beginning of the 21 st century, the string landscape emerged as a mechanism for varying essentially all the constants, including the number of spatial dimensions.The anthropic idea that fundamental parameters are selected from a multitude of different possibilities (each actual in some universe or other) contrasts with the traditional hope of physicists for a theory of everything having no free parameters: as Einstein said, "What really interests me is whether God had any choice in the creation of the world." Quite recently, proponents of the leading candidate for a "theory of everything", string theory, proclaimed "the end of the anthropic principle since there would be no free parameters to select. Ironically, string theory now seems to offer no hope of predicting fundamental parameters, and now some who advocate it invoke the anthropic principle as well.

The modern form of a design argument is put forth by Intelligent design. Proponents of intelligent design often cite the fine-tuning observations that (in part) preceded the formulation of the anthropic principle by Carter as a proof of an intelligent designer. Opponents of intelligent design are not limited to those who hypothesize that other universes exist; they may also argue, anti-anthropically, that the universe is less fine-tuned than often claimed, or that accepting fine tuning as a brute fact is less 
astonishing than the idea of an intelligent creator. Furthermore, even accepting fine tuning, Sober (2005) and Ikeda and Jefferys, argue that the Anthropic Principle as conventionally stated actually undermines intelligent design; see fine-tuned universe.

Don Page criticized the entire theory of cosmic inflation. He emphasized those initial conditions which made possible a thermodynamic arrow of time in a universe with a Big Bang origin, must include the assumption that at the initial singularity, the entropy of the universe was low and therefore extremely improbable. Paul Davies rebutted this criticism by invoking an inflationary version of the anthropic principle. While Davies accepted the premise that the initial state of the visible Universe (which filled a microscopic amount of space before inflating) had to possess a very low entropy value = due to random quantum fluctuations - to account for the observed thermodynamic arrow of time, he deemed this fact an advantage for the theory. That the tiny patch of space from which our observable Universe grew had to be extremely orderly, to allow the post-inflation universe to have an arrow of time, makes it unnecessary to adopt any "ad hoc" hypotheses about the initial entropy state, hypotheses other Big Bang theories require.

\section{String theory and vacuum energy}

String theory predicts a large number of possible universes, called the "backgrounds" or "vacua." The set of these vacua is often called the "multiverse" or "anthropic landscape" or "string landscape." Leonard Susskind has argued that the existence of a large number of vacua puts anthropic reasoning on firm ground: only universes whose properties are such as to allow observers to exist are observed, while a possibly much larger set of universes lacking such properties go unnoticed.

Steven Weinberg believes the Anthropic Principle may be appropriated by cosmologists committed to nontheism, and refers to that Principle as a "turning point" in modern science because applying it to the string landscape "...may explain how the constants of nature that we observe can take values suitable for life without being fine-tuned by a benevolent creator." Others, most notably David Gross but also Lubos Motl, Peter Woit, and Lee Smolin, argue that this is not predictive. Max Tegmark, Mario Livio, and Martin Rees $^{\complement}$ argue that only some aspects of a physical theory need be observable and/or testable for the theory to be accepted, and that many well-accepted theories are far from completely testable at present.

When water freezes into ice, the ice floats because ice is less dense than liquid water. This is one possible example of the anthropic principle, because if ice did not float, it might have been difficult or impossible for living organisms to have existed in water; without the insulating properties of a top ice layer, lakes and ponds would tend to freeze solid and thaw very little during warmer periods. This principle has been criticized as neglecting the existence of the tropical zone and other warmer climates.

Ice is unusual in that it is approximately 9\% less dense than liquid water. Water is the only known nonmetallic substance to expand when it freezes. The density of ice is $0.9167 \mathrm{~g} / \mathrm{cm}^{3}$ at $0^{\circ} \mathrm{C}$, whereas water has a density of $0.9998 \mathrm{~g} / \mathrm{cm}^{3}$ at the same temperature. Liquid water is densest, essentially $1.00 \mathrm{~g} / \mathrm{cm}^{3}$, at $4^{\circ} \mathrm{C}$ and becomes less dense as the water molecules begin to form the hexagonal crystals of ice as the freezing point is reached. This is due to hydrogen bonding dominating the intermolecular forces, which results in a packing of molecules less compact in the solid.

\section{The Anthropic Cosmological Principle,Dark Energy and Vacuum Energy:}

Barrow and Tipler carefully distinguish teleological reasoning from eutaxiological reasoning; the former asserts that order must have a consequent purpose; the latter asserts more modestly that order must have a planned cause. They attribute this important but nearly always overlooked distinction to an obscure 1883 book by $L$. E. HicksSeeing little sense in a principle requiring intelligent life to emerge while remaining indifferent to the possibility of its eventual extinction, Barrow and Tipler propose the:"Final anthropic principle (FAP): Intelligent information-processing must come into existence in the Universe, and, once it comes into existence, it will never die out."

FAP places strong constraints on the structure of the universe, constraints developed further in 
Tippler's The Physics of Immortality. One such constraint is that the universe must end in a big crunch, which seems unlikely in view of the tentative conclusions drawn since 1998 about dark energy, based on observations of very distant supernovas.

A common criticism of Carter's SAP is that it is an easy deus ex machina which discourages searches for physical explanations. To quote Penrose again: "it tends to be invoked by theorists whenever they do not have a good enough theory to explain the observed facts."

The anthropic principles implicitly posit that our ability to ponder cosmology at all is contingent on one or more fundamental physical constants having numerical values falling within quite a narrow range, and this is not a trivial tautology; nor is postulating a multiverse. Moreover, working out the consequences of a change in the fundamental constants for the existence of our species is far from trivial, and, as we have seen, can lead to quite unexpected constraints on physical theory. This reasoning does, however, demonstrate that carbonbased life is impossible under these altered fundamental parameters.. Also, the prior distribution of universes as a function of the fundamental constants is easily modified to get any desired result.

\section{"Cosmological constant, Anthropic Principle, Vacuum Energy and Quantum Field}
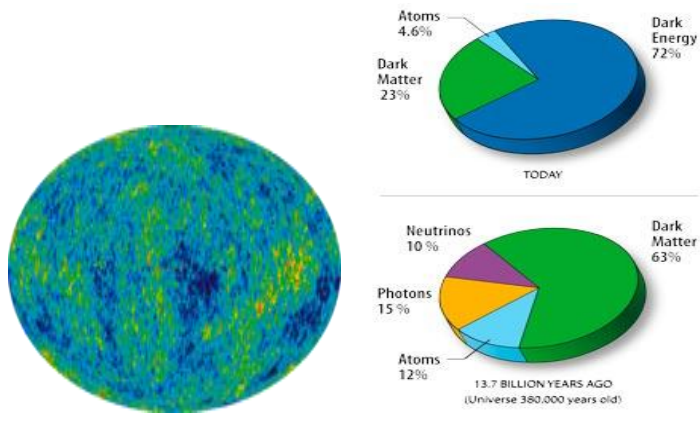

Estimated ratios of dark matter and dark energy (which may be the cosmological constant) in the universe. Dark energy now dominates the energy of the universe, in contrast to earlier epochs when it was insignificant.

In physical cosmology, the cosmological constant (usually denoted by the Greek capital letter lambda: $\Lambda$ ) was proposed by Albert Einstein a modification of his original theory of general relativity to achieve a stationary universe. However, the discovery of cosmic acceleration in $\mathbf{1 9 9 8}$ has renewed interest in a cosmological constant

The cosmological constant $\Lambda$ appears in Einstein's modified field equation in the form of

$$
R_{\mu \nu}-\frac{1}{2} R g_{\mu \nu}+\Lambda g_{\mu \nu}=\frac{8 \pi G}{c^{4}} T_{\mu \nu}
$$

Where $R$ and $g$ pertain to the structure of spacetime, $T$ pertains to matter and energy (thought of as affecting that structure), and $G$ and $c$ are conversion factors that arise from using traditional units of measurement. When $\Lambda$ is zero, this reduces to the original field equation of general relativity. When $T$ is zero, the field equation describes empty space (the vacuum).

The cosmological constant has the same effect as an intrinsic energy density of the vacuum, $\rho_{v a c}$ (and an associated pressure). In this context it is commonly defined with a proportionality factor of $8 \pi: \Lambda=8 \pi \rho_{v a c}$, where unit conventions of general relativity are used (otherwise factors of $G$ and $c$ would also appear). It is common to quote values of energy density directly, though still using the name "cosmological constant". A positive vacuum energy density resulting from a cosmological constant implies a negative pressure, and vice versa. If the energy density is positive, the associated negative pressure will drive an accelerated expansion of empty space In lieu of the cosmological constant itself, cosmologists often refer to the ratio between the 
energy density due to the cosmological constant and the critical density of the universe. This ratio is usually denoted $\Omega_{\Lambda}$. In a flat universe $\Omega_{\Lambda}$ corresponds to the fraction of the energy density of the Universe due to the cosmological constant. Note that this definition is tied to the critical density of the present cosmological era: the critical density changes with cosmological time, but the energy density due to the cosmological constant remains unchanged throughout the history of the universe. Another ratio that is used by scientists is the equation of state which is the ratio of pressure that dark energy puts on the Universe to the energy per unit volume.

\section{VACUUM ENERGY CAUSE OF EXPANSION OF THE UNIVERSE:}

In fact adding the cosmological constant to Einstein's equations does not lead to a static universe at equilibrium because the equilibrium is unstable: if the universe expands slightly, then the expansion releases vacuum energy, which causes yet more expansion. Likewise, a universe which contracts slightly will continue contracting. Empirically, the onslaught of cosmological data in the past decades strongly suggests that our universe has a positive cosmological constant. Finally, it should be noted that some early generalizations of Einstein's gravitational theory, known as classical unified field theories, either introduced a cosmological constant on theoretical grounds or found that it arose naturally from the mathematics. For example, Sir Arthur Stanley Eddington claimed that the cosmological constant version of the vacuum field equation expressed the "epistemological" property that the universe is "self-gauging", and Erwin Schrödinger's pure-affine theory using a simple vibrational principle produced the field equation with a cosmological $t$ distance-redshift relation for Type Ia supernovae indicated that the expansion of the universe is accelerating. When combined with measurements of the cosmic microwave background radiation these implied a value of $\overline{\left.\Omega_{\Lambda} \simeq 0.7,7\right]}$ a result which has been supported and refined by more recent measurements. There are other possible causes of an accelerating universe, such as quintessence, but the cosmological constant is in most respects the simplest solution. Thus, the current standard model of cosmology, the Lambda-CDM model, includes the cosmological constant, which is measured to be on the order of $10^{-52} \mathrm{~m}^{-2}$, in metric units. Multiplied by other constants that appear in the equations, it is often expressed as $10^{-35} \mathrm{~s}^{-2}, 10^{-47} \mathrm{GeV}^{4}, 10^{-29} \mathrm{~g} / \mathrm{cm}^{3}$. In terms of Planck units, and as a natural dimensionless value, the cosmological constant, $\lambda$, is on the order of $10^{-122}$.

As was only recently seen, by works of 't Hooft, Susskind and others, a positive cosmological constant has surprising consequences, such as a finite maximum entropy of the observable universe (see the holographic principle).

A problem arises with inclusion of the cosmological constant in the standard model: i.e., the appearance of solutions with regions of discontinuities (see classification of discontinuities at typical matter density ) Another investigation found the cosmological time, dt, diverges for any finite interval, ds, associated with an observer approaching the cosmological horizon, representing a physical limit to observation for the standard model when the cosmological term is included. This is a key requirement for a complete interpretation / definition of astronomical observations, particularly pertaining to the nature of dark energy and the cosmological constant. All of these findings should be considered major shortcomings of the standard model, but only when the cosmological constant term is included.

\section{ZERO POINT ENERGY OF THE VACUUM AND (E/EB)COSMOLOGICAL CONSTANT -AN UNSOLVED PROBLEM IN PHYSICS}

A major outstanding problem is that most quantum field theories predict a huge value for the quantum vacuum. A common assumption is that the quantum vacuum is equivalent to the cosmological constant. Although no theory exists that supports this assumption, arguments can be made in its favor. Such arguments are usually based on dimensional analysis and effective field theory. If the universe is described by an effective local quantum field theory down to the Planck scale, then we would expect a cosmological constant of the order of $M_{\mathbf{P}}^{4}$. As noted above, the measured cosmological constant is smaller than this by a factor of $10^{-120}$. This discrepancy has been called "the worst theoretical prediction in the history of 
physics!".Some supersymmetric theories require /need a cosmological constant that is exactly zero, which further complicates things. Weinberg explains that if the vacuum energy took different values in different domains of the universe, then observers would necessarily measure values similar to that which is observed: the formation of life-supporting structures would be suppressed in domains where the vacuum energy is much larger. Specifically, if the vacuum energy is negative and its absolute value is substantially larger than it appears to be in the observed universe (say, a factor of 10 larger), holding all other variables (e.g. matter density) constant, that would mean that the universe is closed; furthermore, its lifetime would be shorter than the age of our universe, possibly too short for the intelligent life to form. On the other hand, a universe with a large positive cosmological constant would expand too fast, preventing galaxy formation. According to Weinberg, domains where the vacuum energy are compatible with life would be comparatively rare. Using this argument, Weinberg predicted that the cosmological constant would have a value of less than a hundred times the currently accepted value In 1992 Weinberg refined this prediction of the cosmological constant to 5 to 10 times the matter density.

This argument depends on a lack of a variation of the distribution (spatial or otherwise) in the vacuum energy density, as would be expected if the dark energy were the cosmological constant. There is no evidence that the vacuum energy does vary, but it may be the case if, for example, the vacuum energy is (even in part) the potential of a scalar field such as the residual inflaton (also see quintessence). Another theoretical approach that deals with the issue is that of multiverse theories - predicting a large number of "parallel" universes, possibly with different laws of physics and/or values of fundamental constants. Again, the anthropic principle states that we can only live in one of the universes that is compatible with some form of intelligent life. Critics claim that these theories, when used as an explanation for fine-tuning, commit the inverse gambler's fallacy.

More recent work has suggested the problem may be indirect evidence of a cyclic universe possibly as allowed by string theory. With every cycle of the universe (Big Bang then eventually a Big Crunch) taking about a trillion $\left(10^{12}\right)$ years, "the amount of matter and radiation in the universe is reset, but the cosmological constant is not. Instead, the cosmological constant gradually diminishes over many cycles to the small value observed today." Fecund universes theory, which assumes universes have "offspring" through the creation of black holes, and that these offspring universes have values of physical constants that depend on these of the mother universe. But Lee Smolin assumes that conditions for carbon based life are similar to conditions for black hole creation, which would change the a priori distribution of universes such that universes containing life would be likely. Weak version is mere tautological and the stronger version is epistemological with ontological predication without phenomenological methodologies.

In the context of cosmology the cosmological constant is a homogeneous energy density that causes the expansion of the universe to accelerate. Originally proposed early in the development of general relativity in order to allow a static universe solution it was subsequently abandoned when the universe was found to be expanding. Now the cosmological constant is invoked to explain the observed acceleration of the expansion of the universe. The cosmological constant is the simplest realization of dark energy, which the more generic name is given to the unknown cause of the acceleration of the universe. Its existence is also predicted by quantum physics, where it enters as a form of vacuum energy, although the magnitude predicted by quantum theory does not match that observed in cosmology.

At the time, observations of our universe were limited primarily to stars in our own galaxy, so there was indeed observational evidence justifying the assumption that the universe was static. Einstein's goal was to obtain a Universe that satisfied Mach's principle of the relativity of inertia (for a historical discussion see Pais 1982, Sect 15e), and construct a cosmology that was finite, yet stable against gravitational collapse. The attempt proved futile, as shortly thereafter de Sitter (1917) demonstrated an empty universe solution to Einstein's equations (allowing inertia relative to space empty of matter) and Friedman (1922) derived solutions to Einstein's equations that corresponded to an expanding universe. These results could be considered a prediction that the universe must be expanding or contracting, a remarkable implication of general relativity that was later borne out by observation. When Hubble observationally discovered the 
expansion of the universe Einstein finally abandoned the cosmological constant completely (Einstein 1931).

As of the early 1990s there were tantalizing hints that the cosmological constant might again be needed. The universe appeared to be younger than the oldest stars it contained (age of the stars (e) age of the universe), a feature that was remedied if the universe was currently in an accelerating phase. Number counts of galaxies indicated that the volume contained within a solid angle at high redshift was larger than expected in a decelerating universe. Theoretical arguments from inflation and later observational results from the cosmic microwave background radiation indicated that the universe should be flat, but observations of large scale structure indicated that the matter density was inadequate to achieve this -- vacuum energy could make up the shortfall.

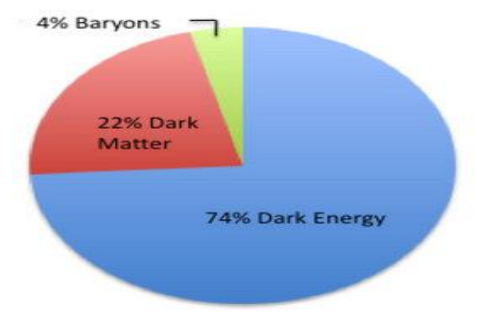

The mass-energy composition of the Universe. The cosmological constant is one possible form $(=)$ of dark energy, which is believed to be behind or produces the acceleration of the expansion Universe. The High-Z supernova team and the Supernova Cosmology project both discovered that high-redshift supernovae were fainter than expected for a decelerating universe and that the difference could be explained/balanced(e) if there was a cosmological constant of just the right magnitude needed to make the universe flat. This was a dramatic convergence of observation and theory. Since then increasingly accurate probes have confirmed to high precision the need for dark energy, (for the production of the flat universe) but the nature of the dark energy is now the issue being investigated. As of 2010 the measured properties of dark energy remain consistent with those of a cosmological constant(implies dark energy=cosmological constant). However, massive observing efforts are underway to test whether this is the correct explanation for the acceleration or whether some other sort of dark energy, perhaps one that changes (e and eb)with time or one that is motivated(eb) by some form of quantum gravity, is needed to (eb)explain the acceleration we see.

\section{The physics of the cosmological constant and Vacuum Energy:}

To explore more deeply the nature of the Universe, we must use the mathematical language in Einstein's general relativity to relate the geometry of space-time (expressed by the metric tensor, $g \mu v$ ) to the energy content of the universe, (expressed by the energy-momentum tensor, $T \boldsymbol{\mu} \boldsymbol{v}$ ). Arguably, one of Einstein's most significant discoveries was that the distribution of energy determines the geometry of spacetime, which is encoded (incorporated)in his field equation,

$R \mu v-12 \operatorname{Rg} \mu \nu=8 \pi G T \mu v,----(1)$

where $G$ is the gravitational constant. Although this is the simplest form of the equations the freedom remains to add a constant term. This "cosmological constant" was what Einstein added in order to achieve a static universe, and it is given the symbol $\Lambda$.

$R \mu v-12 R g \mu v+\Lambda g \mu v=8 \pi G T \mu \nu---(2)$ When $\Lambda$ is positive it acts as a repulsive force.

\section{$\underline{\text { Vacuum Energy }}$}




\begin{tabular}{|lc|}
\hline \multicolumn{2}{|c|}{ Equation of State (w) } \\
\hline Radiation & $1 / 3$ \\
Matter (pressureless) & 0 \\
Curvature & $-1 / 3$ \\
Cosmological Constant & -1 \\
\hdashline Matter (general) & $0<w<1 / 3$ \\
Quintessence & $-1<\mathrm{W}<-1 / 3$ \\
\hline
\end{tabular}

The equation of state, $w$, determines (eb) the relationship between pressure and density in a material according to $w=p / \rho$. Here are some examples of the equation of state for common fluids. When matter is at rest (pressure less dust) it has $w=0$, but as it picks up velocity $(v)$ its equation of state increases until $w \rightarrow 1 / 3$ as $v \rightarrow c$.

\section{VACUUM ENERGY AND UNCERTAINTY PRINCIPLE:}

Vacuum energy arises naturally in quantum mechanics due to the uncertainty principle. In particle physics the vacuum refers $(=)$ to the ground state of the theory -- the lowest energy configuration. The uncertainty principle does not allow e(eb) states of exactly zero energy, even in vacuum (virtual particles are created). Since in general relativity all forms of energy gravitate, this ground state vacuum energy impacts the dynamics of the expansion of the universe.

The early cosmic inflation, when taken along with the recent observations that the universe is currently dominated by a low density vacuum energy, leads to at least two potential problems which modern cosmology must address. First, there is the old cosmological constant problem, with a new twist: the coincidence problem. Secondly, cosmology still lacks a model to predict the observed current cosmic acceleration and to determine whether or not there is a future exit out of this state (as previously in the inflationary case). This constitutes (what is called here) a dynamical problem. In this article a framework is proposed to address these two problems, based on treating the cosmic background vacuum (dark) energy as both dynamical and interacting. The universe behaves as a vacuum-driven cosmic engine which, in search of equilibrium, always back-reacts to vacuum-induced accelerations by increasing its inertia (internal energy) through vacuum energy dissipation. The process couples cosmic vacuum (dark) energy to matter to produce future-directed increasingly comparable amplitudes in these fields by setting up oscillations in the decaying vacuum energy density and corresponding sympathetic ones in the matter fields. By putting bounds on the relative magnitudes of these coupled oscillations the model offers a natural and conceptually simple channel to discuss the coincidence problem, while also suggesting a way to deal with the dynamical problem. A result with useful observational implications is an equation of state $w(t)$ which specifically predicts a variable, quasi-periodic, acceleration for the current universe. This result can be directly tested by future observational techniques such as SNAP.(( Manasse R. Mbonye)

Vacuum energy should not have any dissipative processes such as heat conduction or viscosity, so it should take the form of a perfect fluid,

$T \mu v=(\rho+p) U \mu U v+p g \mu v .(3)$

In order to maintain Lorentz invariance, vacuum energy should also have no preferred direction. Therefore the first term in the perfect fluid energy tensor must be zero, requiring

pvac $=-\rho v a c,(4)$

which corresponds to an equation of state $w v a c=p v a c / \rho v a c=-1$, and results in an energy-momentum tensor for vacuum energy,

$T v a c \mu v=p v a c g \mu v=-\rho v a c g \mu v .(5)$

Equivalence of Cosmological Constant and Vacuum Energy 
We can split the energy-momentum tensor into a term describing the matter and energy, and a term describing the vacuum, $T \mu v=$ Tmatter $\mu v+T v a c \mu v$. Einstein's equation including vacuum energy becomes,

$R \mu v-12 \operatorname{Rg} \mu v=8 \pi G($ Tmatter $\mu v-\rho v a c g \mu v) .(6)$

Recall that the cosmological constant enters Einstein's equation in the form,

$R \mu v-12 R g \mu v+\Lambda g \mu v=8 \pi G T \mu v$.

So vacuum energy and the cosmological constant have identical behaviour in general relativity, as long as the vacuum energy density is identified with,

$\rho v a c=\Lambda 8 \pi G .(7)$

In an homogeneous, isotropic universe the geometry is defined by the Friedman-Lemaittre-Robertson-Walker metric (FLRW metric) and the dynamics of the universe are governed by the Friedman equations (Friedmann equations). The dynamics are driven by the energy content of the universe and the equation of state of the components that make up the energy density. The equation of state relates density $\rho$ to pressure $p$ according to $w=p / \rho$. The cosmological constant enters these equations in the following way, where $a$ is the scale factor of the universe normalized to 1 at the present day, $H=a^{*} / a$ is Hubble's constant (an overdot represents differentiation with respect to time), and $k$ is the curvature of the universe given by $+1,0$, and -1 for positive, flat, and negative curvature respectively,

$H 2=8 \pi G 3 \rho-k a 2+\Lambda 3,(8)$

$a " a=-4 \pi G 3(\rho+3 p)+\Lambda 3 .(9)$

These equations are more concisely written by considering both the cosmological constant and curvature as forms of energy density ( $\rho \Lambda=\Lambda / 8 \pi G$ and $\rho k=-3 k / 8 \pi G a 2)$. Then Eqs. (8) and (9) become

$H 2=8 \pi G 3 \sum i \rho i,(10)$

$a \ddot{a} a=-4 \pi G 3 \sum i(\rho i+3 p i) .(11)$

The different components have different equations of state, $w i$, which determines how their density changes with the expansion of the universe :

$\rho i=\rho i 0 a-3(1+w i)(12)$

Pressure less matter has $w=0$, radiation has $w=1 / 3$, curvature has an effective $w=-1 / 3$, cosmological constant has $w=-1$. (We have used $w \Lambda=-1$, which implies $\rho \Lambda+3 p \Lambda=-2 \rho \Lambda$ in deriving Eq. (11).)

The current energy density of each component, $\rho i 0$, is often represented as a fraction of the critical density, $\rho \mathrm{c}=3 H 20 / 8 \pi G$, which is the energy density required to close the universe (also calculated at the present day). Denoting this $\Omega i=\rho i 0 / \rho \mathrm{c}$ and using Eq. (12) allows us to write

$H 2=H 20 \sum i \rho i \rho c=H 20 \sum i \Omega i a-3(1+w i)(13)$

For pressure to do work there needs to be a pressure gradient -- a relatively high pressure region next to a relatively low pressure region -- that will then cause movement from high pressure to low. In a homogeneous universe there are no pressure gradients, so a positive pressure does no work and has no expanding effect (there are no low-pressure regions for it to push matter into). On the contrary, in general relativity all forms of energy gravitate so pressure effectively pulls, strengthening the attractive force of gravity (thus the factor of $p$ in Eq. (9), which does not appear in Newtonian gravity). The cosmological constant has negative pressure, $w=-1$, so its general relativistic contribution counteracts the normal force of gravity and provides an outwards acceleration. Observational evidence for the accelerating universe is now very strong, with many different experiments covering vastly different timescales, length scales, and physical processes, all supporting the standard $\Lambda \mathrm{CDM}$ cosmological model, in which the universe is flat with an energy density 
made up of about $4 \%$ baryonic matter, $23 \%$ dark matter, and $73 \%$ cosmological constant. For more detail and references see the review by Frieman, Turner and Huterer 2008.

The critical observational result that brought the cosmological constant into its modern prominence was the discovery that distant type Ia supernovae $(0<z<1)$, used as standard candles, were fainter than expected in a decelerating universe (Riess et al. 1998, Perlmutter et al. 1999). Since then many groups have confirmed this result with more supernovae and over a larger range of redshifts. Of particular importance are the observations that extremely high redshift $(\mathrm{z}>1)$ supernovae are brighter than expected, which is the observational signature that is expected from a period of deceleration preceding our current period of acceleration.

\section{Conclusions:}

Under this category, we summarize the work done, namely the study of formulation of the Governing Equations, necessary sine qua non attributions like accentuation and dissipation which are essential functional prerequisite for the consummation and success of the model. It is to be stated that primary focus and locus is of homologues nature and differentially instrumentally activities of the model performance such as stability analysis, asymptotic analysis, solution behavior, and the sententious and pithy prognostications under which the systems become functional or for that matter dysfunctional. We do not write a separate conclusive note. Herein itself is mentioned the holistic and generalizational view of the work done, as has been done in the foregoing. Imperative compatibilities and structural variabilities of the Vacuum Energy and Quantum Field are stated in unequivocal terms. Common patterns of phenomenological methodology, essential predications, suspensional neutralities, rational representations, conferential extrinsicness, interfacial interference and syncopated justice the model does for the generalized goal to be consummated is dealt with. Solutional behavior, stability analyses, asymptotic analysis bear ample testimony, infallible observatory, and impeccable demonstration to the predicational anteriority, character constitution, ontological consonance, primordial exactitude to the acolytish representation and apocryphal aneurism and associated asseveration of the Governing Equations and the obtention of Stability analysis which is dealt in detail ,stating what happens to the singularities, anti generalities or event at contracted points, and normal performance of the model in normal conditions. This helps in the optimum development of the system of governing equations and dynamical improvement of the conditionalities for the instrumental efficaciousness of the stability, or reduction of asymptotic stability as the practical applications demand. No conjecture however is made because of ignorance of such possibilities and possibilities. Concatenated governing equations thus provide a qualitative gradient of internal structural differentiation, and diffuse solidarity abstraction. No separate conclusion statement is made in consideration to the fact that motivational orientation and institutionalization of pattern variables that are used have already been stated here. At Planck's scale there might be energetic franticness or ensorcelled frenzy of the Vacuum Energy and Quantum Field, and these extrapolations have to be explored in detail by more eminent, erudite, and esteemed researchers. Vacuum energy is one type of energy, which could be highly belligerently tempestuous and temerariously reckless when put to different uses. While a tendentious testament is not provided, a first step of a progenitor is taken for the intimate comprehension of the system. Further papers build on this framework towards the consummation of higher theories envisioned. This on the other hand provides a rich receptacle, reliquary repository to other researchers to study practical applications right from the simple appliances like piston to highly sophisticated ones like in CERN

\section{Acknowledgments}

The introduction is a collection of information from, articles, abstracts of the articles, paper reports, home pages of the authors, textbooks, research papers, and various other sources including the internet including Wikipedia. We acknowledge all authors who have contributed to the same. Should there be any act of omission or commission on the part of the authors in not referring to the author, it is authors' 'sincere entreat, earnest beseech ,and fervent appeal to pardon such lapses as has been done or purported to have been done in the foregoing. With great deal of compunction and contrition, the authors beg the pardon of the respective sources. References list is only illustrative and not exhaustive. We have put all concerted efforts 
and sustained endeavors to incorporate the names of all the sources from which information has been extracted. It is because of such eminent, erudite, and esteemed people allowing us to piggy ride on their backs, we have attempted to see little forward, or so we think.

\section{REFERENCES}

[1] Dr K N Prasanna Kumar, Prof B S Kiranagi, Prof C S Bagewadi - MEASUREMENT DISTURBS EXPLANATION OF QUANTUM MECHANICAL STATES-A HIDDEN VARIABLE THEORY - published at: "International Journal of Scientific and Research Publications, Volume 2, Issue 5, May 2012 Edition".

[2] DR K N PRASANNA KUMAR, PROF B S KIRANAGI and PROF C S BAGEWADI -CLASSIC 2 FLAVOUR COLOR SUPERCONDUCTIVITY AND ORDINARY NUCLEAR MATTER-A NEW PARADIGM STATEMENT - published at: "International Journal of Scientific and Research Publications, Volume 2, Issue 5, May 2012 Edition".

[3] A HAIMOVICI: "On the growth of a two species ecological system divided on age groups". Tensor, Vol 37 (1982),Commemoration volume dedicated to Professor Akitsugu Kawaguchi on his $80^{\text {th }}$ birthday

[4] FRTJOF CAPRA: "The web of life" Flamingo, Harper Collins See "Dissipative structures" pages 172-188

[5] HEYLIGHEN F. (2001): "The Science of Self-organization and Adaptivity", in L. D. Kiel, (ed) . Knowledge Management, Organizational Intelligence and Learning, and Complexity, in: The Encyclopedia of Life Support Systems ((EOLSS), (Eolss Publishers, Oxford) [http://www.eolss.net

[6] MATSUI, T, H. Masunaga, S. M. Kreidenweis, R. A. Pielke Sr., W.-K. Tao, M. Chin, and Y. J Kaufman (2006), "Satellitebased assessment of marine low cloud variability associated with aerosol, atmospheric stability, and the diurnal cycle", J. Geophys. Res., 141, D17201， doi:10.1029/2005JD006097

[7] STEVENS, B, G. Feingold, W.R. Cotton and R.L. Walko, "Elements of the microphysical structure of numerically simulated nonprecipitating stratocumulus" J. Atmos. Sci., 53, 980-1006

[8] FEINGOLD, G, Koren, I; Wang, HL; Xue, HW; Brewer, WA (2010), "Precipitation-generated oscillations in open cellular cloud fields" Nature, 166 (7308) 819-852, doi: 10.1038/nature09314, Published 12-Aug 2010

[9] R WOOD "The rate of loss of cloud droplets by coalescence in warm clouds" J.Geophys. Res., 141, doi: 10.1029/2006JD007553, 2006

[10] H. RUND, "The Differential Geometry of Finsler Spaces", Grund. Math. Wiss. Springer-Verlag, Berlin, 195 A. Dold, "Lectures on Algebraic Topology”, 1972, Springer-Verlag

[11] S LEVIN "Some Mathematical questions in Biology vii ,Lectures on Mathematics in life sciences, vol 8" The American Mathematical society, Providence, Rhode island 1976

[12] Furlanetto S. R., Piran T., 2006, Monthly Notices of theRAS, 366, 467

[13] Geller M. J., Huchra J. P., 1989, Science, 246, 897Giovanelli R., Haynes M. P., 1985, Astronomical Journal,90, 2445

[14] Goldberg D. M., Jones T. D., Hoyle F., et al. 2005, Astrophysical Journal, 621, 643

[15] Gottl"ober S., Lokas E. L., Klypin A., Hoffman Y., 2003,Monthly Notices of the RAS, 344, 715

[16] Gregory S. A., Thompson L. A., 1978, Astrophysical Journal, 222, 784

[17] Grogin N. A., Geller M. J., 1999, Astronomical Journal,118, 2561

[18] Grogin N. A., Geller M. J., 2000, Astronomical Journal,119, 32

[19] Gunn J. E., et al. 1998, Astronomical Journal, 116, 3040

[20] Hausman M. A., Olson D. W., Roth B. D., 1983, Astrophysical Journal, 270, 351

[21] Haynes M. P., 2008, in A. H. Bridle, J. J. Condon, \&G. C. Hunt ed., Frontiers of Astrophysics: A Celebration of NRAO's 50th Anniversary Vol. 395 of Astronomical Society of the Pacific Conference Series, H I Cosmology in the Local Universe with ALFALFA. pp 125-+Hoffman Y., Shaham J., 1982, Astrophysical Journal, Letters, 262, L23

[22] Hoyle F., Rojas R. R., Vogeley M. S., Brinkmann J., 2005,Astrophysical Journal, 620, 618

[23] Hoyle F., Vogeley M. S., 2002, Astrophysical Journal, 566,641 
[24] Hoyle F., Vogeley M. S., 2004, Astrophysical Journal, 607,751

[25] Huchra J., Davis M., Latham D., Tonry J., 1983, Astrophysical Journal, Supplement, 52, 89

[26] Hui L., Nicolis A., Stubbs C. W., 2009, Physical Review D,80, 104002

[27] Icke V., 1984, Monthly Notices of the RAS, 206, 1P

[28] Joeveer M., Einasto J., Tago E., 1978, Monthly Notices of the RAS, 185, 357

[29] Jones D. H., et al. 2004, Monthly Notices of the RAS, 355,747

[30] Kamionkowski M., Verde L., Jimenez R., 2009, Journal of Cosmology and Astroparticle Physics, 1, Astrophysical Journal, 339, 595

[31] Plionis M., Basilakos S., 2002, Monthly Notices of the RAS,330, 399

[32] Popescu C. C., Hopp U., Elsaesser H., 1997, Astronomy and Astrophysics, 325, 881Regos E., Geller M. J., 1991, Astrophysical Journal, 377,14

[33] Rojas R. R., Vogeley M. S., Hoyle F., Brinkmann J., 2004,Astrophysical Journal, 617, 50

[34] Rojas R. R., Vogeley M. S., Hoyle F., Brinkmann J., 2005,Astrophysical Journal, 624, 571

[35] Rood H. J., 1988, Annual Review of Astron and Astrophys,26, 245

[36] Ryden B. S., 1995, Astrophysical Journal, 452, 25

[37] Ryden B. S., Melott A. L., 1996, Astrophysical Journal,470, 160

[38] Saunders W., Sutherland W. J., Maddox S. J., et al. 2000,Monthly Notices of the RAS, 317, 55

[39] Shandarin S., Feldman H. A., Heitmann K., Habib S., 2006,

[40] Sheth R. K., van de Weygaert R., 2004,

[41] Strauss M. A., Davis M., Yahil A., Huchra J. P., 1992,Astrophysical Journal, 385, 421

[42] strauss M. A., et al. 2002, Astronomical Journal, 124, 1810

[43] Thuan T. X., Gott III J. R., Schneider S. E., 1987

[44] Gravity and the Quantum Vacuum Inertia HypothesisI. Formalized Groundwork for Extension to GravityAlfonso RuedaDepartment of Electrical Engineering \& Department of Physics, ECS Building California State University, 1250 Bellower Blvd., Long Beach, CA 90840arueda@ csulb.eduBernard Haisch and Roh Tung California Institute for Physics \& Astrophysics

[45] Mitton, S. (2005). Fred Hoyle: A Life in Science. Aurum Press. p. 127

[46] Slipher, V.M (1913). "The Radial Velocity of the Andromeda Nebula". Lowell Observatory Bulletin 1: $56-$ 57. Bibcode 1913LowOB...2...56S..

[47] "People and Discoveries: Big Bang Theory". A Science Odyssey. PBS. Retrieved 2012-03-09.^ Eddington, A. (1931). "The End of the World: from the Standpoint of Mathematical Physics". Nature 127 (3203): 447453. Bibcode 1931 Natur.127..447E.doi:10.1038/127447a0

[48] Milne, E.A. (1935). Relativity, Gravitation and World Structure. Oxford University Press. LCCN 3519093.

[49] Tolman, R.C. (1934). Relativity, Thermodynamics, and Cosmology. Clarendon Press. ISBN 0-486-65383-8.LCCN 3432023.

[50] Yao, W.M. et al (2006). "Review of Particle Physics: Dark Matter". Journal of Physic

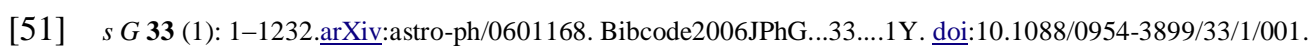


[52] Penrose, R. (1979). "Singularities and Time-Asymmetry". In Hawking, S.W. (Ed); Israel, W. (ed).General Relativity: An Einstein Centenary Survey. Cambridge. pp. 581-638.

[53] Penrose, R. (1989). "Difficulties with Inflationary Cosmology". In Fergus, E.J. (ed). Proceedings of the 14th Texas Symposium on Relativistic Astrophysics. New York Academy of Sciences. pp. 249-264. Doi:10.1111/j.1749-6632.1989.tb50513.x.

First Author: ${ }^{1}$ Mr. K. N.Prasanna Kumar has three doctorates one each in Mathematics, Economics, Political Science. Thesis was based on Mathematical Modeling. He was recently awarded D.litt., for his work on 'Mathematical Models in Political Science'--- Department of studies in Mathematics, Kuvempu University, Shimoga, Karnataka, India

Second Author: ${ }^{2}$ Prof. B.S Kiranagi is the Former Chairman of the Department of Studies in Mathematics, Manasa Gangotri and present Professor Emeritus of UGC in the Department. Professor Kiranagi has guided over 25 students and he has received many encomiums and laurels for his contribution to Co homology Groups and Mathematical Sciences. Known for his prolific writing, and one of the senior most Professors of the country, he has over 150 publications to his credit. A prolific writer and a prodigious thinker, he has to his credit several books on Lie Groups, Co Homology Groups, and other mathematical application topics, and excellent publication history.-- UGC Emeritus Professor (Department of studies in Mathematics), Manasagangotri, University of Mysore, Karnataka, India

Third Author: ${ }^{3}$ Prof. C.S. Bagewadi is the present Chairman of Department of Mathematics and Department of Studies in Computer Science and has guided over 25 students. He has published articles in both national and international journals. Professor Bagewadi specializes in Differential Geometry and its wide-ranging ramifications. He has to his credit more than 159 research papers. Several Books on Differential Geometry, Differential Equations are coauthored by him--- Chairman, Department of studies in Mathematics and Computer science, Jnanasahyadri Kuvempu university, Shankarghatta, Shimoga district, Karnataka, India 